Supporting Information

\title{
Halocyclizations of Unsaturated Sulfoximines
}

\author{
Han Wang, Marcus Frings, and Carsten Bolm* \\ Institute of Organic Chemistry, RWTH Aachen University, Landoltweg 1, 52074 Aachen, \\ Germany
}

\section{Table of Contents}

1. General Information $\quad$ S2

2. Experimental Procedures $\quad$ S2

2.1. General Procedure for the Preparation of $S$-Alkenyl Sulfoximines (1 and 3) $\quad$ S2

2.2. General Procedure for the Preparation of Cyclization Products (2 and 4) S3

2.3. Synthetic Procedure of Compound $5 \quad$ S3

$\begin{array}{lr}\text { 2.4 Synthetic Procedure of Compound } 6 & \text { S3 }\end{array}$

2.5 Method of Opposing Reaction $\quad$ S3

3. References $\quad$ S4

4. Characterization Data $\quad$ S4

4.1.Characterization Data of $S$-Alkenyl Sulfoximines (1 and 3) S4

$\begin{array}{ll}\text { 4.2 Characterization Data of Cyclization Products (2 and } 4) & \text { S8 }\end{array}$

$\begin{array}{ll}4.3 \text { Characterization Data of } 5 \text { and } \mathbf{6} & \text { S14 }\end{array}$

4.4 HPLC Spectra and Characterization Data (2a and 4a) $\quad$ S15

$\begin{array}{lr}\text { 5. }{ }^{1} \mathbf{H} \text { and }{ }^{13} \mathbf{C} \text { NMR Spectra } & \text { S17 }\end{array}$ 


\section{General Information}

Unless otherwise noted, the materials were purchased from commercial suppliers and used without further purification. All the solvents were treated according to general methods. The reactions were monitored by thin layer chromatography (TLC) with aluminium sheets silica gel $60 \mathrm{~F}_{254}$ from Merck, and flash column chromatography purifications were performed using silica gel $60(63-200 \mu \mathrm{m})$ from Merck. ${ }^{1} \mathrm{H}$ and ${ }^{13} \mathrm{C}$ NMR spectra were recorded with an Agilent VNMRS 600, Agilent VNMRS 400 or Varian Mercury 300 in deuterated solvents. Chemical shifts $(\delta)$ are reported in parts per million $(\mathrm{ppm})$ and spin-spin coupling constants $(J)$ are given in $\mathrm{Hz}$, while multiplicities are abbreviated by s (singlet), $\mathrm{d}$ (doublet), t (triplet), q (quartet), br (broad), m (multiplet). The IR spectra were recorded with a PerkinElmer Spectrum 100 spectrometer with an attached UATR device Diamond KRS-5. All IR data were collected by attenuated total reflectance (ATR) and wavenumbers $v$ are given in $\mathrm{cm}^{-}{ }^{1}$. Mass spectra were recorded with a Finnigan SSQ Finnigan 7000 spectrometer (EI, $70 \mathrm{eV}$ ). High resolution mass spectra (HRMS) were recorded on a Thermo Scientific LTQ Orbitrap XL spectrometer. Melting points (mp) were determined on a Büchi B-540 melting point apparatus. Determination and separation of stereoisomers were performed by analytical high-performance liquid chromatography (HPLC) on an Agilent 1200-series with a Chiralpak AD-H column (250 mm $4.6 \mathrm{~mm}$ ) from Chiral Technologies Inc. as chiral stationary phase (CSP) and by preparative supercritical fluid chromatography (SFC) on a Thar SFC Prep 80 from Waters with a Chiralpak IA column $(250 \mathrm{~mm} \cdot 19 \mathrm{~mm})$.

\section{Experimental Procedures}

\subsection{General Procedure for the Preparation of $S$-Alkenyl Sulfoximines \\ $\operatorname{Method~A~}{ }^{\text {S1 }}$}
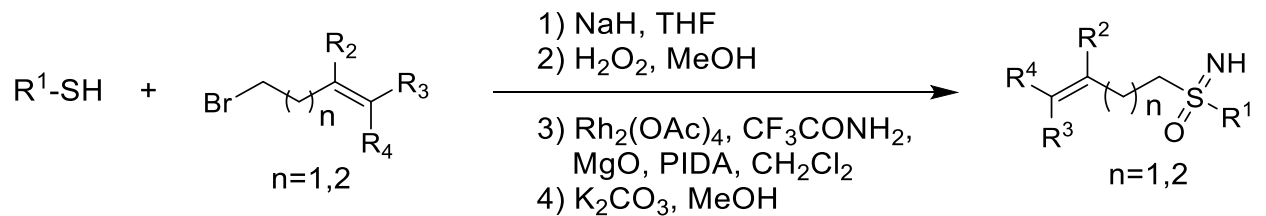

In a flame dried flask under argon, thiophenol $(1.0 \mathrm{mmol})$ was dissolved in dry THF $(10 \mathrm{~mL})$ and cooled to $0{ }^{\circ} \mathrm{C}$. $\mathrm{NaH}(44 \mathrm{mg}, 60 \%$ dispersion in oil, $1.1 \mathrm{mmol}$ ) was added in portions and the slurry was allowed to stir for $15 \mathrm{~min}$. The bromide $(1.05 \mathrm{mmol})$ was then added by syringe. The reaction mixture was allowed to warm to room temperature and stirred until completion (as judged by TLC analysis). The reaction mixture was concentrated, then diluted with $\mathrm{H}_{2} \mathrm{O}(20 \mathrm{~mL})$. The aqueous phase was extracted three times with ethyl acetate. The combined organics were washed with $10 \%$ aqueous $\mathrm{NaOH}$ solution and brine, dried over anhydrous $\mathrm{Na}_{2} \mathrm{SO}_{4}$, filtered, and concentrated to dryness under vacuum. The residue was used in the next step without further purification.

A solution of the residue above in $\mathrm{MeOH}(10 \mathrm{~mL})$ at room temperature was treated with $30 \mathrm{wt} . \%$ aq. $\mathrm{H}_{2} \mathrm{O}_{2}(4.0 \mathrm{mmol}, 0.45 \mathrm{~mL})$, and stirred overnight. After this time, $\mathrm{H}_{2} \mathrm{O}(4.0 \mathrm{~mL})$ was added and the mixture extracted four times with ethyl acetate. The combined organic phases were washed with $\mathrm{H}_{2} \mathrm{O}$ and brine, dried over $\mathrm{Na}_{2} \mathrm{SO}_{4}$, and then concentrated under vacuum. The residue was used in the next step without further purification.

To a suspension of the residue above, trifluoroacetamide (230 mg, $2.0 \mathrm{mmol}), \mathrm{MgO}(161 \mathrm{mg}$, $4.0 \mathrm{mmol})$, and $\mathrm{Rh}_{2}(\mathrm{OAc})_{4}(11 \mathrm{mg}, 2.5 \mathrm{~mol} \%)$ in dry $\mathrm{CH}_{2} \mathrm{Cl}_{2}(10 \mathrm{~mL})$ was added $\mathrm{PhI}(\mathrm{OAc})_{2}(483 \mathrm{mg}$, 
$1.5 \mathrm{mmol}$ ) at room temperature, and the mixture was stirred for $6 \mathrm{~h}$. The resulting suspension was filtered through a pad of celite, and the filtrate was concentrated under vacuum. The residue was used in the next step without further purification.

To the solution of the residue above in $\mathrm{MeOH}(10 \mathrm{~mL})$ was added $\mathrm{K}_{2} \mathrm{CO}_{3}(691 \mathrm{mg}, 5.0 \mathrm{mmol})$ at room temperature, and the mixture was stirred for $30 \mathrm{~min}$. The reaction mixture was concentrated under vacuum, and purification by silica gel column chromatography afforded the $N$-unsubstituted $S$-alkenyl sulfoximines.

\section{Method B $^{\text {S2 }}$}

Following the literature procedure of S2.

\subsection{General Procedure for the Preparation of Cyclization Products.}

To a $15 \mathrm{~mL}$ sealable reaction tube with a magnetic stir bar was added $S$-alkenyl sulfoximine (1 or 3 ) (0.30 mmol), KI (100 mg, $0.60 \mathrm{mmol}), \mathrm{PhI}(\mathrm{OAc})_{2}(145 \mathrm{mg}, 0.45 \mathrm{mmol})$ and $\mathrm{CH}_{2} \mathrm{Cl}_{2}(3.0 \mathrm{~mL})$. The reaction mixture was stirred at room temperature until completion (as judged by TLC analysis). Then the mixture was diluted with $\mathrm{CH}_{2} \mathrm{Cl}_{2}(20 \mathrm{~mL})$ and washed with a saturated aqueous $\mathrm{Na}_{2} \mathrm{~S}_{2} \mathrm{O}_{3}$ solution and brine. The organic layer was concentrated under vacuum. Purification by silica gel column chromatography afforded the cyclization product ( $\mathbf{2}$ or $\mathbf{4})$.

\subsection{Synthetic Procedure of 1-Phenyl-3-[(phenylthio)methyl]-3,4,5,6-tetrahydro-1,2-thiazine-} 1-oxide (5):

To a solution of thiophenol $(55.1 \mathrm{mg}, 0.50 \mathrm{mmol})$ in dry THF $(10 \mathrm{~mL})$, cooled to $0{ }^{\circ} \mathrm{C}$, was added $\mathrm{NaH}$ (22 $\mathrm{mg}, 60 \%$ dispersion in oil, $0.55 \mathrm{mmol}$ ). The reaction mixture was stirred for $15 \mathrm{~min}$ before heterocycle $2 \mathrm{a}(168 \mathrm{mg}, 0.50 \mathrm{mmol})$ was added. Then, the reaction mixture was allowed to warm to room temperature and stirred until completion (as judged by TLC analysis). The resulting mixture was concentrated, and purification by silica gel column chromatography furnished product $\mathbf{5}$.

\subsection{Synthetic Procedure of 3-(Azidomethyl)-1-phenyl-3,4,5,6-tetrahydro-1,2-thiazine- 1-oxide} (6):

Thiazine compound $2 \mathrm{a}(168 \mathrm{mg}, 0.50 \mathrm{mmol})$ was dissolved in DMF (2.0 mL), $\mathrm{NaN}_{3}(39 \mathrm{mg}, 0.60$ mmol) was added, and the reaction mixture was stirred for $24 \mathrm{~h}$. Then $\mathrm{CH}_{2} \mathrm{Cl}_{2}(30 \mathrm{~mL})$ was added, and the reaction mixture was washed with $\mathrm{H}_{2} \mathrm{O}$, dried over $\mathrm{MgSO}_{4}$ and concentrated under reduced pressure. Purification by silica gel column chromatography afforded the corresponding product 6.

\subsection{Method of Opposing Reaction:}

A solution of thiazine compound $2 \mathrm{a}(168 \mathrm{mg}, 0.50 \mathrm{mmol})$ in THF $(3.0 \mathrm{~mL})$ was stirred in a flame-dried Schlenk tube under argon atmosphere. Then, the solution was cooled to $-78{ }^{\circ} \mathrm{C}$ and $n$-BuLi $(0.31 \mathrm{~mL}$, $1.6 \mathrm{M}$ in hexane) was added in drops. The reaction mixture was slowly warmed to room temperature, stirred overnight and quenched with a saturated aqueous $\mathrm{NH}_{4} \mathrm{Cl}$ solution. The organic layer was separated, and the aqueous layer was extracted with $\mathrm{CH}_{2} \mathrm{Cl}_{2}$ three times. The combined extracts were dried over $\mathrm{Na}_{2} \mathrm{SO}_{4}$ and concentrated. Purification by silica gel column chromatography afforded the compound 1a. 


\section{References}

[S1] (a) Coulter, M. M.; Kou, K. G. M.; Galligan, B.; Dong, V. M. J. Am. Chem. Soc. 2010, 132, 16330.

(b) Emerson, C. R.; Zakharov, L. N.; Blakemore, P. R. Chem. Eur. J. 2013, 19, 16342. (c) Okamura, H.; Bolm, C. Org. Lett. 2004, 6, 1305.

[S2] (a) McGrath, M. J.; Bolm, C. Beilstein J. Org. Chem. 2007, 3, 33. (b) Yadav, M. R.; Rit, R. K.; Sahoo, A. K. Chem. Eur. J. 2012, 18, 5541.

\section{Characterization Data}

\subsection{Characterization Data of $S$-Alkenyl Sulfoximines (1 and 3) $S$-(Pent-4-en-1-yl)-S-phenylsulfoximine (1a):}

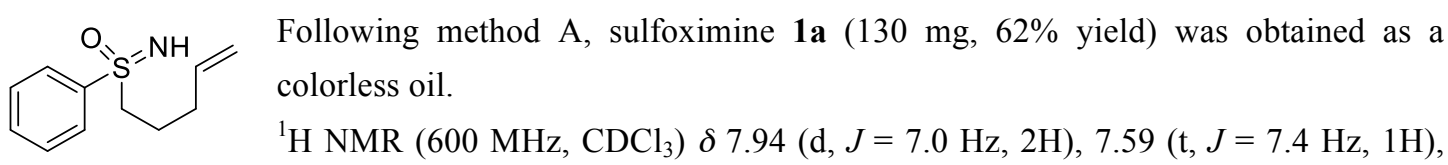
$7.53(\mathrm{t}, J=7.6 \mathrm{~Hz}, 2 \mathrm{H}), 5.69-5.59(\mathrm{~m}, 1 \mathrm{H}), 4.96-4.94(\mathrm{~m}, 2 \mathrm{H}), 3.18-3.07(\mathrm{~m}, 2 \mathrm{H}), 2.84(\mathrm{~s} b r, 1 \mathrm{H})$, $2.07(\mathrm{q}, J=7.0 \mathrm{~Hz}, 2 \mathrm{H}), 1.88-1.70(\mathrm{~m}, 2 \mathrm{H}) .{ }^{13} \mathrm{C}\left\{{ }^{1} \mathrm{H}\right\} \mathrm{NMR}\left(150 \mathrm{MHz}, \mathrm{CDCl}_{3}\right) \delta 136.4,133.1,129.2$, 128.4, 116.3, 56.7, 32.0, 22.2. MS (EI): $m / z=210$ (17), $209\left(2, \mathrm{M}^{+}\right), 140$ (6), 125 (100), 109 (1), 77 (58). IR (ATR): $v=3267,3075,2923,2322,1630,1439,1213,1102,976,741$. HRMS $\mathrm{m} / \mathrm{z}$ : calcd for $\left[\mathrm{C}_{11} \mathrm{H}_{15} \mathrm{NOS}+\mathrm{H}\right]^{+}: 210.0947$, found: 210.0946 .

\section{$S$-(4-Bromophenyl)-S-(pent-4-en-1-yl)sulfoximine (1b):}

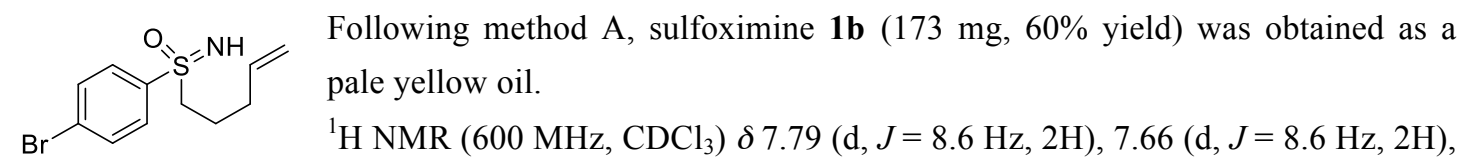
$5.69-5.60(\mathrm{~m}, 1 \mathrm{H}), 4.97-4.95(\mathrm{~m}, 2 \mathrm{H}), 3.16-3.04(\mathrm{~m}, 2 \mathrm{H}), 2.81(\mathrm{~s} \mathrm{br}, 1 \mathrm{H}), 2.08(\mathrm{q}, J=7.2 \mathrm{~Hz}, 2 \mathrm{H})$, $1.86-1.69(\mathrm{~m}, 2 \mathrm{H}) .{ }^{13} \mathrm{C}\left\{{ }^{1} \mathrm{H}\right\} \mathrm{NMR}\left(150 \mathrm{MHz}, \mathrm{CDCl}_{3}\right) \delta 141.0,136.2,132.4,130.0,128.4,116.5,56.7$, 31.9, 22.2. MS (EI): $m / z=288$ (92), 287 (5, $\left.\mathrm{M}^{+}\right), 218$ (1), 205 (13), 203(13), 159 (3), 76 (3). IR $(\mathrm{ATR}): v=3268,3078,2929,2323,1640,1460,1214,1084,982,746$. HRMS $\mathrm{m} / \mathrm{z}$ : calcd for $\left[\mathrm{C}_{11} \mathrm{H}_{14} \mathrm{BrNOS}+\mathrm{H}\right]^{+}:$288.0052, found: 288.0049 .

\section{$S$-(4-Chlorophenyl)-S-(pent-4-en-1-yl)sulfoximine (1c):}<smiles>C=CCCCS(=N)(=O)c1ccc(Cl)cc1</smiles>

Following method A, sulfoximine 1c (154 mg, 63\% yield) was obtained as a colorless oil.

${ }^{1} \mathrm{H}$ NMR $\left(600 \mathrm{MHz}, \mathrm{CDCl}_{3}\right) \delta 7.81(\mathrm{~d}, J=8.5 \mathrm{~Hz}, 2 \mathrm{H}), 7.42(\mathrm{~d}, J=8.4 \mathrm{~Hz}, 2 \mathrm{H})$, $5.61-5.54(\mathrm{~m}, 1 \mathrm{H}), 4.90-4.88(\mathrm{~m}, 2 \mathrm{H}), 3.08-2.98(\mathrm{~m}, 2 \mathrm{H}), 2.85(\mathrm{~s} b r, 1 \mathrm{H}), 2.01(\mathrm{q}, J=7.1 \mathrm{~Hz}, 2 \mathrm{H})$, $1.76-1.63(\mathrm{~m}, 2 \mathrm{H}) .{ }^{13} \mathrm{C}\left\{{ }^{1} \mathrm{H}\right\} \mathrm{NMR}\left(150 \mathrm{MHz}, \mathrm{CDCl}_{3}\right) \delta 140.5,139.6,136.3,129.9,129.3,116.3,56.7$, 31.9, 22.2. MS (EI): $m / z=244$ (5), 174 (2), 159 (17), 143 (2), 111 (36). IR (ATR): $v=3267,3087$, 2926, 2320, 1573, 1395, 1213, 1091, 974, 75. HRMS $m / z$ : calcd for $\left[\mathrm{C}_{11} \mathrm{H}_{14} \mathrm{ClNOS}+\mathrm{H}\right]^{+}: 244.0557$, found: 244.0553 . 


\section{$S$-(p-Tolyl)-S-(pent-4-en-1-yl)sulfoximine (1d):}

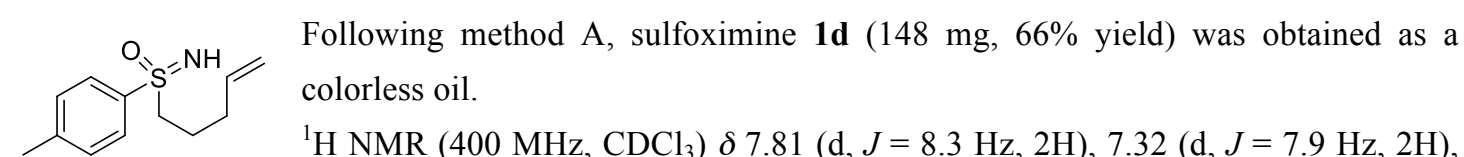
$5.72-5.57(\mathrm{~m}, 1 \mathrm{H}), 4.97-4.93(\mathrm{~m}, 2 \mathrm{H}), 3.10(\mathrm{ddd}, J=9.8,6.0,3.5 \mathrm{~Hz}, 2 \mathrm{H}), 2.50(\mathrm{~s} \mathrm{br}, 1 \mathrm{H}), 2.42(\mathrm{~s}$, $3 \mathrm{H}), 2.07$ (q, $J=7.2 \mathrm{~Hz}, 2 \mathrm{H}), 1.87-1.69(\mathrm{~m}, 2 \mathrm{H}) .{ }^{13} \mathrm{C}\left\{{ }^{1} \mathrm{H}\right\} \mathrm{NMR}\left(100 \mathrm{MHz}, \mathrm{CDCl}_{3}\right) \delta 143.9,136.4$, 129.8, 128.4, 116.2, 56.9, 32.0, 22.3, 21.5. MS (EI): $m / z=224$ (100), $223\left(19, \mathrm{M}^{+}\right), 139$ (57), 123 (2), 108 (22), 91 (24). IR (ATR): $v=3272,3062,2927,2320,1601,1424,1212,1103,973,745$. HRMS $m / z$ : calcd for $\left[\mathrm{C}_{12} \mathrm{H}_{17} \mathrm{NOS}+\mathrm{H}\right]^{+}: 224.1105$, found: 224.1103 .

\section{$S$-(4-Methoxyphenyl)-S-(pent-4-en-1-yl)sulfoximine (1e):}

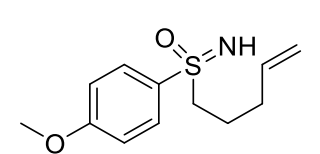

Following method A, sulfoximine 1e (130 mg, 54\% yield) was obtained as a colorless oil.

${ }^{1} \mathrm{H}$ NMR $\left(600 \mathrm{MHz}, \mathrm{CDCl}_{3}\right) \delta 7.86(\mathrm{~d}, J=8.8 \mathrm{~Hz}, 2 \mathrm{H}), 6.98(\mathrm{~d}, J=8.9 \mathrm{~Hz}, 2 \mathrm{H})$, $5.80-5.53(\mathrm{~m}, 1 \mathrm{H}), 4.97-4.94(\mathrm{~m}, 2 \mathrm{H}), 3.86(\mathrm{~s}, 3 \mathrm{H}), 3.15-3.04(\mathrm{~m}, 2 \mathrm{H}), 2.47(\mathrm{~s} \mathrm{br}, 1 \mathrm{H}), 2.07$ (q, $J=$ $7.2 \mathrm{~Hz}, 2 \mathrm{H}), 1.88-1.68(\mathrm{~m}, 2 \mathrm{H}) .{ }^{13} \mathrm{C}\left\{{ }^{1} \mathrm{H}\right\}$ NMR $\left(150 \mathrm{MHz}, \mathrm{CDCl}_{3}\right) \delta 163.3,136.5,133.3,130.5$, 116.2, 114.3, 57.1, 55.6, 32.0, 22.4. MS (EI): $m / z=240$ (52), 239 (5, $\left.\mathrm{M}^{+}\right), 170$ (9), 155 (100), 139 (2), 123 (27), 107 (5). IR (ATR): $v=3270,3076,2936,2291,1588,1487,1222,1102,984,746$. HRMS $m / z$ : calcd for $\left[\mathrm{C}_{12} \mathrm{H}_{17} \mathrm{NO}_{2} \mathrm{~S}+\mathrm{H}\right]^{+}: 240.1053$, found: 240.1053 .

\section{$S$-(Naphthalen-2-yl)-S-(pent-4-en-1-yl)sulfoximine (1f):}

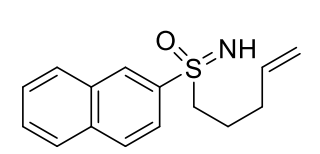

Following method A, sulfoximine 1f (234 $\mathrm{mg}, 65 \%$ yield) was obtained as a colorless oil.

${ }^{1} \mathrm{H}$ NMR $\left(600 \mathrm{MHz}, \mathrm{CDCl}_{3}\right) \delta 8.53(\mathrm{~s}, 1 \mathrm{H}), 7.99(\mathrm{~d}, J=8.8 \mathrm{~Hz}, 2 \mathrm{H}), 7.96-$ $7.90(\mathrm{~m}, 2 \mathrm{H}), 7.69-7.59(\mathrm{~m}, 2 \mathrm{H}), 5.70-5.60(\mathrm{~m}, 1 \mathrm{H}), 4.97-4.92(\mathrm{~m}, 2 \mathrm{H}), 3.28-3.16(\mathrm{~m}, 2 \mathrm{H}), 2.70$ (s br, 1H), 2.10 (q, $J=7.1 \mathrm{~Hz}, 2 \mathrm{H}), 1.92-1.75(\mathrm{~m}, 2 \mathrm{H}) .{ }^{13} \mathrm{C}\left\{{ }^{1} \mathrm{H}\right\} \mathrm{NMR}\left(150 \mathrm{MHz}, \mathrm{CDCl}_{3}\right) \delta 138.8$, 136.4, 135.0, 132.3, 129.9, 129.5, 129.3, 129.0, 127.9, 127.6, 123.4, 116.3, 56.8, 32.0, 22.3. MS (EI): $m / z=260$ (84), 259 (16, M $\left.{ }^{+}\right), 244$ (2), 190 (4), 175 (100), 127 (69). IR (ATR): $v=3266,3064$, 2927, 2320, 1635, 1438, 1215, 1089, 985, 743. HRMS $\mathrm{m} / \mathrm{z}$ : calcd for $\left[\mathrm{C}_{15} \mathrm{H}_{17} \mathrm{NOS}+\mathrm{Na}\right]^{+}: 282.0923$, found: 282.0923 .

\section{$S$-Cyclohexyl-S-(pent-4-en-1-yl)sulfoximine (1g):}

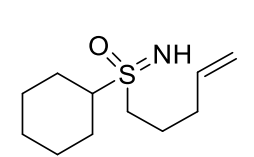

Following method A, sulfoximine $1 \mathrm{~g}$ ( $91 \mathrm{mg}, 42 \%$ yield) was obtained as a colorless oil.

${ }^{1} \mathrm{H}$ NMR $\left(600 \mathrm{MHz}, \mathrm{CDCl}_{3}\right) \delta 5.75(\mathrm{ddt}, J=16.9,10.2,6.7 \mathrm{~Hz}, 1 \mathrm{H}), 5.10-4.99(\mathrm{~m}$, 2H), $2.99-2.91(\mathrm{~m}, 2 \mathrm{H}), 2.85(\mathrm{tt}, J=12.2,3.5 \mathrm{~Hz}, 1 \mathrm{H}), 2.43(\mathrm{~s} \mathrm{br}, 1 \mathrm{H}), 2.24-2.08(\mathrm{~m}, 4 \mathrm{H}), 1.98-$ $1.89(\mathrm{~m}, 4 \mathrm{H}), 1.72(\mathrm{~d}, J=12.9 \mathrm{~Hz}, 1 \mathrm{H}), 1.50(\mathrm{qt}, J=12.6,3.3 \mathrm{~Hz}, 2 \mathrm{H}), 1.30-1.34(\mathrm{~m}, 2 \mathrm{H}), 1.21(\mathrm{tt}, J$ $=13.0,3.4 \mathrm{~Hz}, 1 \mathrm{H}) \cdot{ }^{13} \mathrm{C}\left\{{ }^{1} \mathrm{H}\right\} \mathrm{NMR}\left(150 \mathrm{MHz}, \mathrm{CDCl}_{3}\right) \delta 136.5,116.4,62.5,50.3,32.4,25.6,25.3,25.2$, 25.1, 20.9. MS (EI): $m / z=216$ (62), 215 (7, $\left.\mathrm{M}^{+}\right), 146$ (2), 131 (3), 83 (71), 69 (74). IR (ATR): $v=3264$, $3073,2931,2318,1636,1445,1201,1101,978$. HRMS $m / z$ : calcd for $\left[\mathrm{C}_{11} \mathrm{H}_{21} \mathrm{NOS}+\mathrm{H}\right]^{+}: 216.1417$, found: 216.1420 . 
$S$-(Benzyl)-S-(pent-4-en-1-yl)sulfoximine (1h):

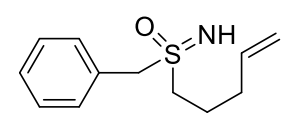

Following method A, sulfoximine 1 h (105 $\mathrm{mg}, 47 \%$ yield) was obtained as a colorless oil.

${ }^{1} \mathrm{H}$ NMR (600 MHz, $\left.\mathrm{CDCl}_{3}\right) \delta 7.41(\mathrm{~s}, 5 \mathrm{H}), 5.74(\mathrm{ddt}, J=17.0,10.2,6.7 \mathrm{~Hz}, 1 \mathrm{H})$, $5.12-5.00(\mathrm{~m}, 2 \mathrm{H}), 4.29(\mathrm{~m}, 2 \mathrm{H}), 3.11-2.89(\mathrm{~m}, 2 \mathrm{H}), 2.46$ (s br, 1H), 2.19 (q, $J=7.2 \mathrm{~Hz}, 2 \mathrm{H}), 1.98$ (dtt, $J=14.4,7.1,5.0 \mathrm{~Hz}, 2 \mathrm{H}) .{ }^{13} \mathrm{C}\left\{{ }^{1} \mathrm{H}\right\} \operatorname{NMR}\left(150 \mathrm{MHz}, \mathrm{CDCl}_{3}\right) \delta 136.3,130.9,129.1,129.0,127.8$, 116.5, 62.0, 52.4, 32.2, 21.5. MS (EI): $m / z=223\left(4, \mathrm{M}^{+}\right), 139$ (6), 132 (4), 123 (3), 91 (100), 77 (1). IR (ATR): $v=3267, \quad 2934, \quad 2309, \quad 1627, \quad 1435, \quad 1212, \quad 985, \quad 697$. HRMS $m / z$ : calcd for $\left[\mathrm{C}_{12} \mathrm{H}_{17} \mathrm{NOS}+\mathrm{Na}\right]^{+}: 246.0923$, found: 246.0928 .

\section{$S$-(Methyl)- $S$-(pent-4-en-1-yl)sulfoximine (1i):}

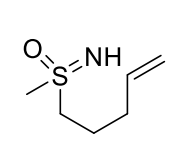

Following method B, sulfoximine $1 \mathrm{i}$ ( $57 \mathrm{mg}, 39 \%$ yield) was obtained as a colorless oil. ${ }^{1} \mathrm{H}$ NMR $\left(600 \mathrm{MHz}, \mathrm{CDCl}_{3}\right) \delta 5.76(\mathrm{ddt}, J=17.0,10.2,6.7 \mathrm{~Hz}, 1 \mathrm{H}), 5.10-5.03(\mathrm{~m}, 2 \mathrm{H})$, $3.09-3.05(\mathrm{~m}, 2 \mathrm{H}), 2.96(\mathrm{~s}, 3 \mathrm{H}), 2.21(\mathrm{q}, J=7.1 \mathrm{~Hz}, 2 \mathrm{H}), 1.96(\mathrm{ddt}, J=11.4,8.5,6.3 \mathrm{~Hz}$, 2H). ${ }^{13} \mathrm{C}\left\{{ }^{1} \mathrm{H}\right\} \mathrm{NMR}\left(150 \mathrm{MHz}, \mathrm{CDCl}_{3}\right) \delta 136.3,116.6,56.4,43.0,32.1,22.2$. MS (EI): $m / z=148$ (78), $147\left(2, \mathrm{M}^{+}\right), 132$ (3), 78 (3), 69 (14), 62 (2). IR (ATR): $v=3269$, 2925, 1727, 1641, 1429, 1205, 1007, 924, 759. HRMS $m / z$ : calcd for $\left[\mathrm{C}_{6} \mathrm{H}_{13} \mathrm{NOS}+\mathrm{Na}\right]^{+}: 170.0610$, found: 170.0602 .

\section{$S$-(Hex-4-en-1-yl)-S-phenylsulfoximine (1j):}

Following method A, sulfoximine $\mathbf{1 j}(140 \mathrm{mg}, 63 \%$ yield $)$ was obtained as a
colorless oil.
${ }_{1}^{1} \mathrm{H}$ NMR $\left(600 \mathrm{MHz}, \mathrm{CDCl}_{3}\right) \delta 7.90(\mathrm{~d}, J=7.3 \mathrm{~Hz}, 2 \mathrm{H}), 7.56(\mathrm{t}, J=7.3 \mathrm{~Hz}, 1 \mathrm{H})$, $7.49(\mathrm{t}, J=7.6 \mathrm{~Hz}, 2 \mathrm{H}), 5.38-5.16(\mathrm{~m}, 2 \mathrm{H}), 3.14-3.00(\mathrm{~m}, 2 \mathrm{H}), 2.65(\mathrm{~s} \mathrm{br}, 1 \mathrm{H}), 1.95(\mathrm{q}, J=6.7 \mathrm{~Hz}$, $2 \mathrm{H}), 1.78-1.61(\mathrm{~m}, 2 \mathrm{H}), 1.54(\mathrm{dd}, J=6.3,1.5 \mathrm{~Hz}, 3 \mathrm{H}) .{ }^{13} \mathrm{C}\left\{{ }^{1} \mathrm{H}\right\} \mathrm{NMR}\left(150 \mathrm{MHz}, \mathrm{CDCl}_{3}\right) \delta 142.0$, 132.9, 129.1, 128.9, 128.3, 126.9, 56.8, 30.8, 22.8, 17.8. MS (EI): $m / z=224$ (100), $223\left(19, \mathrm{M}^{+}\right), 140$ (21), 125 (31), 109 (1), 83 (3), 77 (17). IR (ATR): $v=3268,2930,2323,11444,1214,1097,968,740$. HRMS $m / z$ : calcd for $\left[\mathrm{C}_{12} \mathrm{H}_{17} \mathrm{ClNOS}+\mathrm{Na}\right]^{+}: 246.0923$, found: 246.0927 .

\section{$S$-(4-Methylpent-4-en-1-yl)-S-phenylsulfoximine (1k)}

Following method $\mathrm{A}$, sulfoximine 1k $(134 \mathrm{mg}, 60 \%$ yield $)$ was obtained as a
colorless oil. (t, $J=7.6 \mathrm{~Hz}, 2 \mathrm{H}), 4.66(\mathrm{~d}, J=65.0 \mathrm{~Hz}, 2 \mathrm{H}), 3.16-3.05(\mathrm{~m}, 2 \mathrm{H}), 2.66(\mathrm{~s} \mathrm{br}, 1 \mathrm{H}), 2.04$ (t, $J=7.0 \mathrm{~Hz}$, $2 \mathrm{H}), 1.92-1.77(\mathrm{~m}, 2 \mathrm{H}), 1.62(\mathrm{~s}, 3 \mathrm{H}) .{ }^{13} \mathrm{C}\left\{{ }^{1} \mathrm{H}\right\} \mathrm{NMR}\left(150 \mathrm{MHz}, \mathrm{CDCl}_{3}\right) \delta 143.5,133.0,129.1,128.4$, 127.8, 111.5, 56.8, 35.9, 21.9, 20.8. MS (EI): $m / z=224$ (100), 223 (2, $\left.\mathrm{M}^{+}\right), 208$ (2), 140 (21), 125 (32), 109 (2), 77 (23). IR (ATR): $v=3264,2935,2322,1706,1444,1214,1081,974,732$. HRMS $m / z$ : calcd for $\left[\mathrm{C}_{12} \mathrm{H}_{17} \mathrm{NOS}+\mathrm{Na}\right]^{+}: 246.0923$, found: 246.0918 .

\section{$S$-(5-Methylhex-4-en-1-yl)-S-phenylsulfoximine (11):}

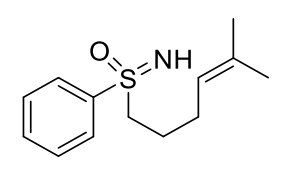

Following method A, sulfoximine 11 (154 $\mathrm{mg}, 65 \%$ yield) was obtained as a colorless oil.

${ }^{1} \mathrm{H}$ NMR (600 MHz, $\left.\mathrm{CDCl}_{3}\right) \delta 7.96(\mathrm{dd}, J=8.4,1.3 \mathrm{~Hz}, 2 \mathrm{H}), 7.61(\mathrm{t}, J=7.4 \mathrm{~Hz}$, $1 \mathrm{H}), 7.54$ (t, $J=7.6 \mathrm{~Hz}, 2 \mathrm{H}), 4.97$ (ddt, $J=8.7,5.7,1.4 \mathrm{~Hz}, 1 \mathrm{H}), 3.16-3.07$ (m, 2H), 2.65 (s br, 1H), 2.02 (q, $J=7.2 \mathrm{~Hz}, 2 \mathrm{H}), 1.76-1.67(\mathrm{~m}, 2 \mathrm{H}), 1.65$ (s, 3H), 1.54 (s, $3 \mathrm{H}) .{ }^{13} \mathrm{C}\left\{{ }^{1} \mathrm{H}\right\}$ 
NMR (150 MHz, $\left.\mathrm{CDCl}_{3}\right) \delta 142.0,133.5,132.9,129.1,128.4,122.2,57.0,26.4,25.7,23.3,17.7 . \mathrm{MS}$ (EI): $m / z=238$ (75), $237\left(7, \mathrm{M}^{+}\right), 140$ (25), 125(52), 109 (4), 97 (18), 77 (34). IR (ATR): $v=3265$, 2923, 2320, 1707, 1443, 1213, 1088, 976, 743. HRMS $m / z$ : calcd for $\left[\mathrm{C}_{13} \mathrm{H}_{19} \mathrm{NOS}+\mathrm{Na}\right]^{+}: 260.1080$, found: 260.1084 .

\section{$S$-(But-3-en-1-yl)-S-phenylsulfoximine (3a):}

Following method A, sulfoximine 3a $(129 \mathrm{mg}, 66 \%$ yield $)$ was obtained as a
colorless oil.
${ }^{1} \mathrm{H}$ NMR $\left(400 \mathrm{MHz}, \mathrm{CDCl}_{3}\right) \delta 7.95(\mathrm{~d}, J=7.3 \mathrm{~Hz}, 2 \mathrm{H}), 7.60(\mathrm{t}, J=7.3 \mathrm{~Hz}, 1 \mathrm{H})$, $7.53(\mathrm{t}, J=7.5 \mathrm{~Hz}, 2 \mathrm{H}), 5.69$ (ddt, $J=16.8,10.2,6.5 \mathrm{~Hz}, 1 \mathrm{H}), 5.07-4.95$ (m, 2H), 3.19 (ddd, $J=9.7$, 6.1, 4.7 Hz, 2H), $2.54-2.37$ (m, 3H). ${ }^{13} \mathrm{C}\left\{{ }^{1} \mathrm{H}\right\}$ NMR (100 MHz, $\left.\mathrm{CDCl}_{3}\right) \delta 141.7,133.9,133.1,129.2$, 128.4, 117.0, 56.5, 27.3. MS (EI): $m / z=196$ (44), $195\left(1, \mathrm{M}^{+}\right), 140$ (2), 125 (100), 109 (38), 77 (59), 55 (28). IR (ATR): $v=3241,2932,2336,1639,1446,1206,1094,985,743$. HRMS m/z: calcd for $\left[\mathrm{C}_{10} \mathrm{H}_{13} \mathrm{NOS}+\mathrm{Na}\right]^{+}: 218.0610$, found: 218.0611 .

\section{$S$-(4-Bromophenyl)-S-(but-3-en-1-yl)sulfoximine (3b):}

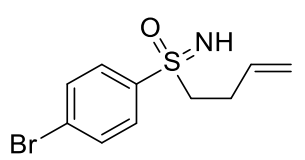

Following method A, sulfoximine 3b (166 mg, 61\% yield) was obtained as a pale yellow oil.

${ }^{1} \mathrm{H}$ NMR (400 MHz, $\left.\mathrm{CDCl}_{3}\right) \delta 7.81(\mathrm{~d}, J=8.6 \mathrm{~Hz}, 2 \mathrm{H}), 7.67(\mathrm{~d}, J=8.6 \mathrm{~Hz}, 2 \mathrm{H})$, 5.69 (ddt, $J=16.8,10.2,6.5 \mathrm{~Hz}, 1 \mathrm{H}), 5.20-4.86(\mathrm{~m}, 2 \mathrm{H}), 3.21-3.14(\mathrm{~m}, 2 \mathrm{H})$, $2.51-2.36(\mathrm{~m}, 3 \mathrm{H}) .{ }^{13} \mathrm{C}\left\{{ }^{1} \mathrm{H}\right\} \mathrm{NMR}\left(100 \mathrm{MHz}, \mathrm{CDCl}_{3}\right) \delta 133.7,132.5,130.0,128.4,117.2,56.5,27.3$. MS (EI): $m / z=274$ (3), 218 (3), 203 (64), 187 (4), 157 (15), 155(15), 55 (100). IR (ATR): $v=3266$, $2927,2315,1569,1217,1086,983,819,743$. HRMS $m / z$ : calcd for $\left[\mathrm{C}_{10} \mathrm{H}_{12} \mathrm{BrNOS}+\mathrm{Na}\right]^{+}: 295.9715$, found: 295.9718 .

\section{$S$-(But-3-en-1-yl)-S-(4-chlorophenyl)sulfoximine (3c):}

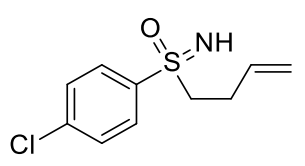

Following method A, sulfoximine 3c (158 mg, 69\% yield) was obtained as a colorless oil.

${ }^{1} \mathrm{H}$ NMR (400 MHz, $\left.\mathrm{CDCl}_{3}\right) \delta 7.89(\mathrm{~d}, J=8.6 \mathrm{~Hz}, 2 \mathrm{H}), 7.51(\mathrm{~d}, J=8.6 \mathrm{~Hz}, 2 \mathrm{H})$, $5.69(\mathrm{ddt}, J=16.8,10.2,6.5 \mathrm{~Hz}, 1 \mathrm{H}), 5.15-4.92(\mathrm{~m}, 2 \mathrm{H}), 3.32-3.07$ (m, 2H), $2.54-2.38(\mathrm{~m}, 2 \mathrm{H}), 2.21(\mathrm{~s} b r, 1 \mathrm{H}) .{ }^{13} \mathrm{C}\left\{{ }^{1} \mathrm{H}\right\}$ NMR $\left(100 \mathrm{MHz}, \mathrm{CDCl}_{3}\right) \delta 139.9,133.7,129.9,129.5$, 117.2, 56.6, 27.3. MS (EI): $m / z=230$ (10), 229 (1, $\left.\mathrm{M}^{+}\right), 214$ (2), 174 (6), 159 (100), 143 (2), 111 (26), 55 (57). IR (ATR): $v=3080,2927,2318,1571,1387,1216,1089,988,752$. HRMS m/z: calcd for $\left[\mathrm{C}_{10} \mathrm{H}_{12} \mathrm{ClNOS}+\mathrm{Na}\right]^{+}: 252.0220$, found: 252.0215 .

\section{$S$-(But-3-en-1-yl)-S-(p-tolyl)sulfoximine (3d):}

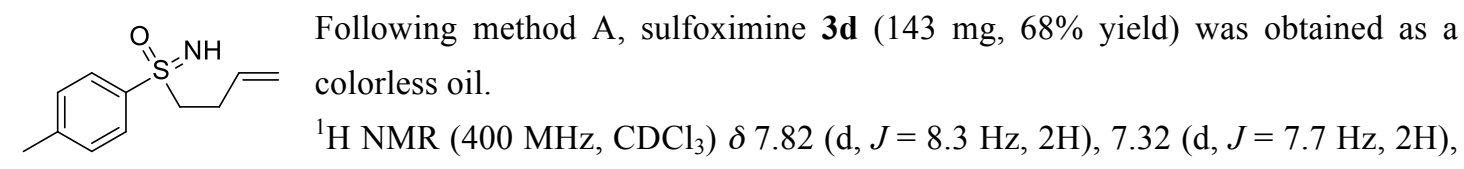
$5.69-5.67$ (m, 1H), $5.07-4.95(\mathrm{~m}, 2 \mathrm{H}), 3.27-3.07(\mathrm{~m}, 2 \mathrm{H}), 2.71$ (s br, 1H), $2.51-2.34(\mathrm{~m}, 5 \mathrm{H})$. ${ }^{13} \mathrm{C}\left\{{ }^{1} \mathrm{H}\right\} \mathrm{NMR}\left(100 \mathrm{MHz}, \mathrm{CDCl}_{3}\right) \delta 144.0,138.6,134.0,129.8,128.5,116.9,56.6,27.4,21.5 . \mathrm{MS}$ (EI): $m / z=210$ (29), 209 (2, $\mathrm{M}^{+}$), 194 (2), 154 (4), 139 (100), 91 (29). IR (ATR): $v=3271,2923,2313$, $1740,1595,1409,1214,1101,979,811$. HRMS $m / z$ : calcd for $\left[\mathrm{C}_{11} \mathrm{H}_{15} \mathrm{NOS}+\mathrm{H}\right]^{+}: 210.0947$, found: 210.0944 . 


\section{$S$-(But-3-en-1-yl)-S-(4-methoxyphenyl)sulfoximine (3e):}

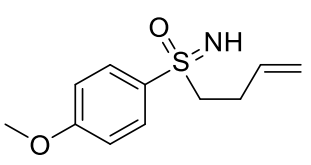

Following method A, sulfoximine $3 \mathrm{e}$ (149 $\mathrm{mg}, 66 \%$ yield) was obtained as a colorless oil.

${ }^{1} \mathrm{H}$ NMR $\left(400 \mathrm{MHz}, \mathrm{CDCl}_{3}\right) \delta 7.87(\mathrm{~d}, J=9.0 \mathrm{~Hz}, 2 \mathrm{H}), 6.98(\mathrm{~d}, J=8.9 \mathrm{~Hz}$, 2H), 5.69 (ddt, $J=16.8,10.1,6.5 \mathrm{~Hz}, 1 \mathrm{H}), 5.07-4.95(\mathrm{~m}, 2 \mathrm{H}), 3.86(\mathrm{~s}, 3 \mathrm{H})$, $3.23-3.11(\mathrm{~m}, 2 \mathrm{H}), 2.62(\mathrm{~s} \mathrm{br}, 1 \mathrm{H}), 2.51-2.35(\mathrm{~m}, 2 \mathrm{H}) .{ }^{13} \mathrm{C}\left\{{ }^{1} \mathrm{H}\right\} \mathrm{NMR}\left(100 \mathrm{MHz}, \mathrm{CDCl}_{3}\right) \delta 163.4$, 134.0, 132.9, 130.6, 116.9, 114.3, 56.8, 55.7, 27.5. MS (EI): $m / z=226$ (28), $225\left(4, \mathrm{M}^{+}\right), 170$ (3), 155 (100), 139 (1), 55 (7). IR (ATR): $v=3276,2934,2311,1737,1588,1489,1221,1100,991,823$. HRMS $m / z$ : calcd for $\left[\mathrm{C}_{11} \mathrm{H}_{15} \mathrm{NO}_{2} \mathrm{~S}+\mathrm{Na}\right]^{+}: 248.0716$, found: 248.0707 .

\section{$S$-(But-3-en-1-yl)-S-(naphthalen-2-yl)sulfoximine (3f):}

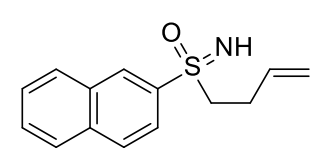

Following method A, sulfoximine $\mathbf{3 f}$ (127 $\mathrm{mg}, 52 \%$ yield) was obtained as a colorless oil.

${ }^{1} \mathrm{H}$ NMR $\left(600 \mathrm{MHz}, \mathrm{CDCl}_{3}\right) \delta 8.19(\mathrm{~s}, 1 \mathrm{H}), 7.98(\mathrm{~d}, J=8.6 \mathrm{~Hz}, 1 \mathrm{H}), 7.97-7.88$ (m, 2H), $7.62-7.56(\mathrm{~m}, 3 \mathrm{H}), 5.80$ (ddt, $J=16.9,10.2,6.6 \mathrm{~Hz}, 1 \mathrm{H}), 5.14-5.04(\mathrm{~m}, 2 \mathrm{H}), 3.01-2.89$ (m, 2H), $2.62-2.52(\mathrm{~m}, 1 \mathrm{H}), 2.39-2.31(\mathrm{~m}, 1 \mathrm{H}) .{ }^{13} \mathrm{C}\left\{{ }^{1} \mathrm{H}\right\} \mathrm{NMR}\left(150 \mathrm{MHz}, \mathrm{CDCl}_{3}\right) \delta 140.7,134.8$, 134.4, 132.9, 129.4, 128.5, 128.0, 127.7, 127.3, 124.8, 119.8, 117.1, 55.8, 26.2. MS (EI): $m / z=246$ (2), 190 (1), 175 (28), 159 (2), 127 (24), 55 (100). IR (ATR): $v=3440,3057,29233,22317,1731,1430$, 1041, 916, 746. HRMS $m / z$ : calcd for $\left[\mathrm{C}_{14} \mathrm{H}_{15} \mathrm{NOS}+\mathrm{Na}\right]^{+}: 268.0767$, found: 268.0771 .

\section{$S$-(But-3-en-1-yl)-S-cyclohexylsulfoximine (3g):}

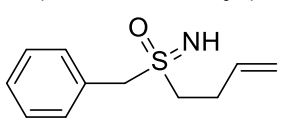

Following method A, sulfoximine $\mathbf{3 g}$ (120 $\mathrm{mg}, 57 \%$ yield) was obtained as a colorless oil.

${ }^{1} \mathrm{H}$ NMR $\left(400 \mathrm{MHz}, \mathrm{CDCl}_{3}\right) \delta 7.39-7.26(\mathrm{~m}, 5 \mathrm{H}), 5.78$ (ddt, $J=16.8,10.2,6.5$ $\mathrm{Hz}, 1 \mathrm{H}), 5.14-5.02(\mathrm{~m}, 2 \mathrm{H}), 4.09-3.89(\mathrm{~m}, 2 \mathrm{H}), 2.63(\mathrm{dd}, J=8.8,7.0 \mathrm{~Hz}, 2 \mathrm{H}), 2.56-2.46(\mathrm{~m}, 2 \mathrm{H})$. ${ }^{13} \mathrm{C}\left\{{ }^{1} \mathrm{H}\right\}$ NMR $\left(150 \mathrm{MHz}, \mathrm{CDCl}_{3}\right) \delta 134.9,130.0,129.0,128.9,128.4,117.1,58.4,50.0,26.6$. MS (EI): $m / z=209\left(4, \mathrm{M}^{+}\right), 139$ (9), 123 (1), 91 (100), 55 (3). IR (ATR): $v=3409,2921,2321,1727,1435$, 1028, 919, 766. HRMS $m / z$ : calcd for $\left[\mathrm{C}_{11} \mathrm{H}_{15} \mathrm{NOS}+\mathrm{Na}\right]^{+}: 232.0767$, found: 232.0767 .

\subsection{Characterization Data of Cyclization Products (2 and 4)}

\section{3-(Iodomethyl)-1-phenyl-3,4,5,6-tetrahydro-1,2-thiazine-1-oxide (2a):}

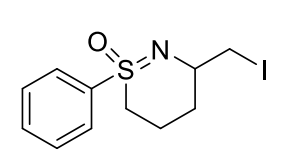

Following the general procedure, 1a provided the corresponding cyclized product 2a (92 mg, 92\% yield) as a light yellow viscous oil.

mixture.

The diastereomeric ratio (77:23) was determined by ${ }^{1} \mathrm{H}$ NMR of the crude product

${ }^{1} \mathrm{H}$ NMR $\left(600 \mathrm{MHz}, \mathrm{CDCl}_{3}\right.$, mixture of diastereomers) $\delta 8.14(\mathrm{~d}, J=8.0 \mathrm{~Hz}, 2 \mathrm{H}), 7.98(\mathrm{~d}, J=7.4 \mathrm{~Hz}$, $0.7 \mathrm{H}), 7.66-7.60(\mathrm{~m}, 1.4 \mathrm{H}), 7.58-7.51(\mathrm{~m}, 2.7 \mathrm{H}), 3.90-3.85(\mathrm{~m}, 0.4 \mathrm{H}), 3.64-3.60(\mathrm{~m}, 0.4 \mathrm{H}), 3.54$ (t, $J=10.2 \mathrm{~Hz}, 0.4 \mathrm{H}), 3.47-3.43(\mathrm{~m}, 3 \mathrm{H}), 3.13(\mathrm{dt}, J=11.8,3.4 \mathrm{~Hz}, 1 \mathrm{H}), 3.09-3.01(\mathrm{~m}, 0.7 \mathrm{H}), 2.77$ (ddd, $J=13.7,11.9,4.6 \mathrm{~Hz}, 1 \mathrm{H}), 2.64-2.54(\mathrm{~m}, 1 \mathrm{H}), 2.51-2.46(\mathrm{~m}, 0.4 \mathrm{H}), 2.26(\mathrm{dp}, J=14.9,3.9 \mathrm{~Hz}$, 1H), $2.16-2.10(\mathrm{~m}, 0.4 \mathrm{H}), 2.04-1.99(\mathrm{~m}, 0.8 \mathrm{H}), 1.97-1.91(\mathrm{~m}, 1 \mathrm{H}), 1.55(\mathrm{dtt}, J=13.4,10.3,3.2 \mathrm{~Hz}$, 1H). ${ }^{13} \mathrm{C}\left\{{ }^{1} \mathrm{H}\right\}$ NMR $\left(150 \mathrm{MHz}, \mathrm{CDCl}_{3}\right.$, mixture of diastereomers) $\delta 139.7,139.2,133.5,133.3,129.2$, $129.2,128.8,128.1,56.7,52.3,51.3,50.6,29.7,25.3,20.4,19.6,17.1,12.2$. MS (EI): $m / z=335(21$, 
$\mathrm{M}^{+}$), 208 (85), 194 (100), 109 (6), 125 (52), 77 (14). IR (ATR): $v=3059,2930,2327,1730,1442$, $1224,1123,882,739$. HRMS $m / z$ : calcd for $\left[\mathrm{C}_{11} \mathrm{H}_{14} \mathrm{INOS}+\mathrm{H}\right]^{+}: 335.9914$, found: 335.9908 .

\section{1-(4-Bromophenyl)-3-(iodomethyl)-3,4,5,6-tetrahydro-1,2-thiazine-1-oxide (2b):}<smiles>O=[SH]1(c2ccc(Br)cc2)CCCC(CI)N1</smiles>

Following the general procedure, $\mathbf{1 b}$ provided the corresponding cyclized product $\mathbf{2 b}$ (102 $\mathrm{mg}, 82 \%$ yield) as a light brown viscous oil.

The diastereomeric ratio (72:28) was determined by ${ }^{1} \mathrm{H}$ NMR of the crude

${ }^{1} \mathrm{H}$ NMR $\left(600 \mathrm{MHz}, \mathrm{CDCl}_{3}\right.$, mixture of diastereomers) $\delta 8.01(\mathrm{~d}, J=8.6 \mathrm{~Hz}, 2 \mathrm{H}), 7.86(\mathrm{~d}, J=8.6 \mathrm{~Hz}$, $0.9 \mathrm{H}), 7.70(\mathrm{dd}, J=8.6,5.9 \mathrm{~Hz}, 2.9 \mathrm{H}), 3.89$ (dq, $J=9.3,4.6 \mathrm{~Hz}, 0.5 \mathrm{H}), 3.64-3.60(\mathrm{~m}, 0.5 \mathrm{H}), 3.52(\mathrm{t}$, $J=10.2 \mathrm{~Hz}, 0.5 \mathrm{H}), 3.47-3.41(\mathrm{~m}, 3 \mathrm{H}), 3.15(\mathrm{dt}, J=11.9,3.5 \mathrm{~Hz}, 1 \mathrm{H}), 3.12-3.08(\mathrm{~m}, 0.5 \mathrm{H}), 3.00$ (ddd, $J=12.4,10.8,4.7 \mathrm{~Hz}, 0.5 \mathrm{H}), 2.76(\mathrm{ddd}, J=13.7,11.8,4.6 \mathrm{~Hz}, 1 \mathrm{H}), 2.64-2.54(\mathrm{~m}, 1 \mathrm{H}), 2.51-$ $2.45(\mathrm{~m}, 0.5 \mathrm{H}), 2.31-2.24(\mathrm{~m}, 1 \mathrm{H}), 2.18-2.11(\mathrm{~m}, 0.5 \mathrm{H}), 2.05-1.99(\mathrm{~m}, 1 \mathrm{H}), 1.93(\mathrm{dd}, J=13.6,3.5$ $\mathrm{Hz}, 1 \mathrm{H}), 1.63-1.51(\mathrm{~m}, 1 \mathrm{H}) .{ }^{13} \mathrm{C}\left\{{ }^{1} \mathrm{H}\right\}$ NMR $\left(150 \mathrm{MHz}, \mathrm{CDCl}_{3}\right.$, mixture of diastereomers) $\delta 138.8$, 138.3, 132.5, 132.4, 130.4, 129.7, 128.8, 128.7, 56.7, 2.3, 51.3, 50.7, 29.6, 25.4, 20.3, 19.7, 17.1, 12.1.MS (EI): $m / z=415$ (16), $413\left(16, \mathrm{M}^{+}\right), 286$ (67), 274 (92), 272 (100), 203 (54), 155 (8). IR (ATR): $v=3075, \quad 2935, \quad 2323, \quad 1732, \quad 1460,1226, \quad 1125, \quad 749$. HRMS $m / z$ : calcd for $\left[\mathrm{C}_{11} \mathrm{H}_{13} \mathrm{BrINOS}+\mathrm{H}\right]^{+}:$413.9019, found: 413.9015 .

\section{1-(4-Chlorophenyl)-3-(iodomethyl)-3,4,5,6-tetrahydro-1,2-thiazine-1-oxide (2c):}<smiles>O=[SH]1(c2ccc(Cl)cc2)CCCC(CI)N1</smiles>

Following the general procedure, 1c provided the corresponding cyclized product $2 \mathbf{c}(95 \mathrm{mg}, 86 \%$ yield) as a light brown viscous oil.

The diastereomeric ratio (73:27) was determined by ${ }^{1} \mathrm{H}$ NMR of the crude

${ }^{1} \mathrm{H}$ NMR (400 MHz, $\mathrm{CDCl}_{3}$, mixture of diastereomers) $\delta 8.05(\mathrm{~d}, J=8.7 \mathrm{~Hz}, 2 \mathrm{H}), 7.89(\mathrm{~d}, J=8.7 \mathrm{~Hz}$, $0.5 \mathrm{H}), 7.53-7.46(\mathrm{~m}, 2.5 \mathrm{H}), 3.87-3.78(\mathrm{~m}, 0.3 \mathrm{H}), 3.62-3.53(\mathrm{~m}, 0.3 \mathrm{H}), 3.50(\mathrm{~d}, J=10.1 \mathrm{~Hz}, 0.3 \mathrm{H})$, $3.46-3.33(\mathrm{~m}, 3 \mathrm{H}), 3.11$ (dt, $J=11.7,3.4 \mathrm{~Hz}, 1 \mathrm{H}), 3.08-2.91$ (m, $0.5 \mathrm{H}), 2.72$ (ddd, $J=13.8,11.7$, $4.6 \mathrm{~Hz}, 1 \mathrm{H}), 2.62-2.47(\mathrm{~m}, 1 \mathrm{H}), 2.46-2.41(\mathrm{~m}, 0.3 \mathrm{H}), 2.23(\mathrm{dtt}, J=14.4,4.4,3.3 \mathrm{~Hz}, 1 \mathrm{H}), 2.11-$ $2.06(\mathrm{~m}, 0.3 \mathrm{H}), 2.01-1.96(\mathrm{~m}, 0.5 \mathrm{H}), 1.93-1.86(\mathrm{~m}, 1 \mathrm{H}), 1.60-1.46(\mathrm{~m}, 1 \mathrm{H}) .{ }^{13} \mathrm{C}\left\{{ }^{1} \mathrm{H}\right\}$ NMR $(100$ $\mathrm{MHz}, \mathrm{CDCl}_{3}$, mixture of diastereomers) $\delta 140.2,140.1,138.3,137.8,130.3,129.7,129.5,129.4,56.6$, 52.3, 51.4, 50.7, 29.6, 25.3, 20.3, 19.6, 17.1, 12.0. MS (EI): $m / z=369$ (25, $\left.\mathrm{M}^{+}\right), 242$ (94), 228 (100), 159 (74), 111 (11). IR (ATR): $v=3075,2933,2323,1736,1464,1226,1111,1009,758$. HRMS $m / z$ : calcd for $\left[\mathrm{C}_{11} \mathrm{H}_{13} \mathrm{CIINOS}+\mathrm{H}\right]^{+}: 369.9524$, found: 369.9520 .

\section{3-(Iodomethyl)-1-(p-tolyl)-3,4,5,6-tetrahydro-1,2-thiazine-1-oxide (2d):}

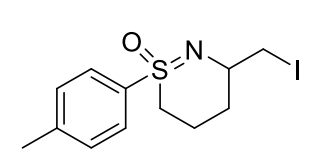

Following the general procedure, 1d provided the corresponding cyclized product $\mathbf{2 d}$ ( $97 \mathrm{mg}, 93 \%$ yield) as a light yellow viscous oil.

The diastereomeric ratio $(78: 22)$ was determined by ${ }^{1} \mathrm{H}$ NMR of the crude

${ }^{1} \mathrm{H}$ NMR (600 MHz, $\mathrm{CDCl}_{3}$, mixture of diastereomers) $\delta 7.99(\mathrm{dd}, J=8.4,2.3 \mathrm{~Hz}, 2 \mathrm{H}), 7.84(\mathrm{dd}, J=$ $8.4,2.3 \mathrm{~Hz}, 0.6 \mathrm{H}), 7.36-7.30(\mathrm{~m}, 2.6 \mathrm{H}), 3.90-3.81(\mathrm{~m}, 0.3 \mathrm{H}), 3.66-3.59(\mathrm{~m}, 0.3 \mathrm{H}), 3.54$ (ddd, $J=$ 12.8, 9.4, $2.6 \mathrm{~Hz}, 0.3 \mathrm{H}), 3.49-3.37(\mathrm{~m}, 3 \mathrm{H}), 3.10(\mathrm{dd}, J=11.8,2.9 \mathrm{~Hz}, 1 \mathrm{H}), 3.06-2.92(\mathrm{~m}, 0.6 \mathrm{H})$, $2.80-2.70(\mathrm{~m}, 1 \mathrm{H}), 2.63-2.52(\mathrm{~m}, 1 \mathrm{H}), 2.50-2.46(\mathrm{~m}, 0.3 \mathrm{H}), 2.43(\mathrm{~s}, 4 \mathrm{H}), 2.29-2.22(\mathrm{~m}, 1 \mathrm{H}), 2.15$ $-2.10(\mathrm{~m}, 0.3 \mathrm{H}), 2.04-1.99(\mathrm{~m}, 0.6 \mathrm{H}), 1.96-1.91(\mathrm{~m}, 1 \mathrm{H}), 1.59-1.47(\mathrm{~m}, 1 \mathrm{H}) .{ }^{13} \mathrm{C}\left\{{ }^{1} \mathrm{H}\right\}$ NMR $(150$ 
$\mathrm{MHz}, \mathrm{CDCl}_{3}$, mixture of diastereomers) $\delta 144.4,144.3,136.6,136.2,129.8,129.8,128.8,128.1,56.6$, 52.3, 51.4, 50.7, 29.7, 25.2, 21.6, 21.6, 20.4, 19.7, 17.1, 12.2. MS (EI): $m / z=349\left(21, \mathrm{M}^{+}\right), 222(81)$, 208 (100), 139 (46), 91 (9). IR (ATR): $v=2931,2321,1734,1432,1224,1120,1015,805,714$. HRMS $m / z$ : calcd for $\left[\mathrm{C}_{12} \mathrm{H}_{16} \mathrm{INOS}+\mathrm{H}\right]^{+}: 350.0070$, found: 350.0070 .

\section{3-(Iodomethyl)-1-(4-methoxyphenyl)-3,4,5,6-tetrahydro-1,2-thiazine-1-oxide (2e):}<smiles>COc1ccc([SH]2(=O)CCCC(CI)N2)cc1</smiles>
Following the general procedure, 1e provided the corresponding cyclized product 2 e (92 $\mathrm{mg}, 89 \%$ yield) as a light yellow viscous oil.

The diastereomeric ratio $(75: 25)$ was determined by ${ }^{1} \mathrm{H}$ NMR of the crude product mixture.

${ }^{1} \mathrm{H}$ NMR (600 MHz, $\mathrm{CDCl}_{3}$, mixture of diastereomers) $\delta 8.03(\mathrm{dd}, J=9.2,2.6 \mathrm{~Hz}, 2 \mathrm{H}), 7.89(\mathrm{dd}, J=$ 9.1, 2.6 Hz, 0.6H), $7.04-6.95(\mathrm{~m}, 2.6 \mathrm{H}), 3.87-3.83(\mathrm{~m}, 4.2 \mathrm{H}), 3.63-3.59(\mathrm{~m}, 0.3 \mathrm{H}), 3.54(\mathrm{td}, J=$ $10.1,2.7 \mathrm{~Hz}, 0.3 \mathrm{H}), 3.44-3.38(\mathrm{~m}, 3 \mathrm{H}), 3.10(\mathrm{dt}, J=11.9,3.2 \mathrm{~Hz}, 1 \mathrm{H}), 3.07-3.05(\mathrm{~m}, 0.3 \mathrm{H}), 2.99-$ $2.94(\mathrm{~m}, 0.3 \mathrm{H}), 2.75-2.73(\mathrm{~m}, 1 \mathrm{H}), 2.58-2.53(\mathrm{~m}, 1 \mathrm{H}), 2.48-2.42(\mathrm{~m}, 0.3 \mathrm{H}), 2.26-2.20(\mathrm{~m}, 1 \mathrm{H})$, $2.14-2.12(\mathrm{~m}, 0.3 \mathrm{H}), 2.00-1.98(\mathrm{~m}, 0.6 \mathrm{H}), 1.93-1.88(\mathrm{~m}, 1 \mathrm{H}), 1.56-1.47(\mathrm{~m}, 1 \mathrm{H}) .{ }^{13} \mathrm{C}\left\{{ }^{1} \mathrm{H}\right\} \mathrm{NMR}$ (150 MHz, $\mathrm{CDCl}_{3}$, mixture of diastereomers) $\delta 163.7,163.6,130.9,130.6,130.4,114.4,56.6,55.7$, 52.3, 51.7, 51.0, 29.7, 25.1, 20.5, 19.9, 17.0, 12.2. MS (EI): $m / z=365$ (14, $\left.\mathrm{M}^{+}\right), 238$ (95), $224(100)$, 155 (56), 139 (7). IR (ATR): $v=2934,2847,2321,1736,1587,1231,1115,1021,818,719$. HRMS $m / z$ : calcd for $\left[\mathrm{C}_{12} \mathrm{H}_{16} \mathrm{INO}_{2} \mathrm{~S}+\mathrm{H}\right]^{+}: 366.0019$, found: 366.0023 .

\section{3-(Iodomethyl)-1-(naphthalen-2-yl)-3,4,5,6-tetrahydro-1,2-thiazine-1-oxide (2f):}<smiles>O=[SH]1(c2ccc3ccccc3c2)CCCC(CI)N1</smiles>

Following the general procedure, 1f provided the corresponding cyclized product $\mathbf{2 f}$ ( $89 \mathrm{mg}, 77 \%$ yield) as a light yellow viscous oil.

The diastereomeric ratio $(82: 18)$ was determined by ${ }^{1} \mathrm{H}$ NMR of the crude product mixture.

${ }^{1} \mathrm{H}$ NMR (400 MHz, $\mathrm{CDCl}_{3}$, mixture of diastereomers) $\delta 8.69(\mathrm{~d}, J=1.8 \mathrm{~Hz}, 1 \mathrm{H}), 8.55(\mathrm{~d}, J=1.8 \mathrm{~Hz}$, $0.2 \mathrm{H}), 8.09(\mathrm{dd}, J=8.7,1.9 \mathrm{~Hz}, 1 \mathrm{H}), 8.02-7.94(\mathrm{~m}, 2.4 \mathrm{H}), 7.95-7.85(\mathrm{~m}, 1.4 \mathrm{H}), 7.69-7.52(\mathrm{~m}$, $2.4 \mathrm{H}), 3.97-3.88(\mathrm{~m}, 0.2 \mathrm{H}), 3.65(\mathrm{dd}, J=9.9,4.5 \mathrm{~Hz}, 0.2 \mathrm{H}), 3.57(\mathrm{t}, J=10.2 \mathrm{~Hz}, 0.2 \mathrm{H}), 3.51-3.41$ $(\mathrm{m}, 3 \mathrm{H}), 3.16(\mathrm{dt}, J=11.8,3.4 \mathrm{~Hz}, 1 \mathrm{H}), 3.12-3.04(\mathrm{~m}, 0.4 \mathrm{H}), 2.89-2.77(\mathrm{~m}, 1 \mathrm{H}), 2.61(\mathrm{qt}, J=13.7$, $3.2 \mathrm{~Hz}, 1 \mathrm{H}), 2.53-2.47(\mathrm{~m}, 0.2 \mathrm{H}), 2.31-2.22(\mathrm{~m}, 1 \mathrm{H}), 2.19-2.11(\mathrm{~m}, 0.2 \mathrm{H}), 2.07-2.02(\mathrm{~m}, 0.4 \mathrm{H})$, $2.00-1.92(\mathrm{~m}, 1 \mathrm{H}), 1.66-1.52(\mathrm{~m}, 1 \mathrm{H}) .{ }^{13} \mathrm{C}\left\{{ }^{1} \mathrm{H}\right\} \mathrm{NMR}\left(100 \mathrm{MHz}, \mathrm{CDCl}_{3}\right.$, mixture of diastereomers $)$ $\delta 136.0,135.2$, 132.2, 130.7, 123.0, 129.6, 129.5, 129.4, 129.4, 129.1, 129.1, 127.9, 127.6, 127.4, 123.5, $122.8,56.8,52.4,51.2,50.6,29.7,25.4,20.4,19.6,17.2,12.3 . \mathrm{MS}(\mathrm{EI}): m / z=385\left(80, \mathrm{M}^{+}\right), 258(72)$, 244 (100), 175 (44), 127 (14). IR (ATR): $v=3056,2928,2323,1731,1348,1221,1119,808,728$. HRMS $m / z$ : calcd for $\left[\mathrm{C}_{15} \mathrm{H}_{16} \mathrm{INOS}+\mathrm{H}\right]^{+}: 386.0070$, found: 386.0070 .

\section{1-Cyclohexyl-3-(iodomethyl)-3,4,5,6-tetrahydro-1,2-thiazine-1-oxide (2g):}<smiles>O=[SH]1(C2CCCCC2)CCCC(CI)N1</smiles>

Following the general procedure, $\mathbf{1 g}$ provided the corresponding cyclized product $2 \mathrm{~g}$ (69 $\mathrm{mg}, 68 \%$ yield) as a brown viscous oil.

The diastereomeric ratio (76:24) was determined by ${ }^{1} \mathrm{H}$ NMR of the crude product mixture.

${ }^{1} \mathrm{H}$ NMR (600 MHz, $\mathrm{CDCl}_{3}$, mixture of diastereomers) $\delta 3.64(\mathrm{dt}, J=6.5,3.7 \mathrm{~Hz}, 0.1 \mathrm{H}), 3.52(\mathrm{~s}, 0.1 \mathrm{H})$, $3.42(\mathrm{td}, J=10.4,1.9 \mathrm{~Hz}, 0.1 \mathrm{H}), 3.40-3.30(\mathrm{~m}, 1 \mathrm{H}), 3.32-3.26(\mathrm{~m}, 1 \mathrm{H}), 3.23-3.18(\mathrm{~m}, 1 \mathrm{H}), 2.87-$ $2.82(\mathrm{~m}, 1 \mathrm{H}), 2.81-2.77(\mathrm{~m}, 1 \mathrm{H}), 2.67-2.60(\mathrm{~m}, 1 \mathrm{H}), 2.42(\mathrm{qd}, J=13.8,3.0 \mathrm{~Hz}, 1 \mathrm{H}), 2.34-2.23(\mathrm{~m}$, 
$2 \mathrm{H}), 2.23-2.18(\mathrm{~m}, 1 \mathrm{H}), 2.17-2.13(\mathrm{~m}, 0.1 \mathrm{H}), 2.06-2.03(\mathrm{~m}, 0.1 \mathrm{H}), 2.00-1.98(\mathrm{~m}, 0.1 \mathrm{H}), 1.95-$ $1.87(\mathrm{~m}, 3 \mathrm{H}), 1.73-1.68(\mathrm{~m}, 1 \mathrm{H}), 1.53-1.44(\mathrm{~m}, 2 \mathrm{H}), 1.34-1.24(\mathrm{~m}, 3 \mathrm{H}), 1.23-1.16(\mathrm{~m}, 1 \mathrm{H})$. ${ }^{13} \mathrm{C}\left\{{ }^{1} \mathrm{H}\right\}$ NMR $\left(150 \mathrm{MHz}, \mathrm{CDCl}_{3}\right.$, mixture of diastereomers) $\delta 62.2,62.2,56.0,52.6,43.8,42.2,30.1$, $26.2,25.9,25.5,25.3,25.3,25.3,25.2,25.2,25.1,24.9,24.7,19.6,18.0,16.2,12.3$. MS (EI): $\mathrm{m} / z=$ $341\left(19, \mathrm{M}^{+}\right), 214$ (25), 200 (38), 131 (4), 83 (58). IR (ATR): $v=2929,2326,1736,1444,1209,1106$, $1006,883,728$. HRMS $m / z$ : calcd for $\left[\mathrm{C}_{11} \mathrm{H}_{20} \mathrm{INOS}+\mathrm{H}\right]^{+}: 342.0383$, found: 342.0382 .

\section{1-Benzyl-3-(iodomethyl)-3,4,5,6-tetrahydro-116,2-thiazine-1-oxide (2h):}

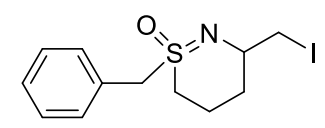

Following the general procedure, $\mathbf{1 h}$ provided the corresponding cyclized product $\mathbf{2 h}$ ( $81 \mathrm{mg}, 77 \%$ yield) as a pale yellow viscous oil.

The diastereomeric ratio $(75: 25)$ was determined by ${ }^{1} \mathrm{H}$ NMR of the crude

product mixture.

${ }^{1} \mathrm{H}$ NMR (600 MHz, $\mathrm{CDCl}_{3}$, mixture of diastereomers) $\delta 7.48-7.44(\mathrm{~m}, 2.2 \mathrm{H}), 7.41-7.36(\mathrm{~m}, 5 \mathrm{H})$, $4.39-4.11(\mathrm{~m}, 2.9 \mathrm{H}), 3.65-3.59(\mathrm{~m}, 0.5 \mathrm{H}), 3.49(\mathrm{ddd}, J=9.9,4.5,1.4 \mathrm{~Hz}, 0.5 \mathrm{H}), 3.43-3.38(\mathrm{~m}$, $0.5 \mathrm{H}), 3.38-3.21(\mathrm{~m}, 3 \mathrm{H}), 2.84(\mathrm{ddd}, J=13.0,8.6,4.5 \mathrm{~Hz}, 0.5 \mathrm{H}), 2.68(\mathrm{dt}, J=11.5,3.5 \mathrm{~Hz}, 1 \mathrm{H}), 2.54$ $(\mathrm{ddd}, J=13.5,11.6,5.1 \mathrm{~Hz}, 1.5 \mathrm{H}), 2.41-2.32(\mathrm{~m}, 1 \mathrm{H}), 2.29-2.21(\mathrm{~m}, 0.5 \mathrm{H}), 2.10(\mathrm{dt}, J=14.5,4.0$ $\mathrm{Hz}, 1 \mathrm{H}), 1.91-1.72(\mathrm{~m}, 3 \mathrm{H}), 1.26-1.14(\mathrm{~m}, 1 \mathrm{H}) .{ }^{13} \mathrm{C}\left\{{ }^{1} \mathrm{H}\right\}$ NMR $\left(150 \mathrm{MHz}, \mathrm{CDCl}_{3}\right.$, mixture of diastereomers) $\delta 131.1,130.7,129.1,129.0,129.0,128.8,128.5,128.2,61.3,60.3,57.5,53.3,45.0$, 44.0, 29.8, 26.2, 19.5, 18.0, 17.3, 12.8. MS (EI): $m / z=349$ (1, $\left.\mathrm{M}^{+}\right), 222$ (3), 208 (14), 139 (4), 91 (100). IR (ATR): $v=3429,2927,2323,1731,1426,1194,1084,1011,873,697$. HRMS m/z: calcd for $\left[\mathrm{C}_{12} \mathrm{H}_{16} \mathrm{INOS}+\mathrm{Na}\right]^{+}: 371.9889$, found: 371.9882 .

\section{3-(Iodomethyl)-1-methyl-3,4,5,6-tetrahydro-116,2-thiazine-1-oxide (2i):}

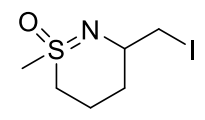

Following the general procedure, $\mathbf{1 i}$ provided the corresponding cyclized product $\mathbf{2} \mathbf{i}$ (41 mg, 50\% yield) as a light brown viscous oil.

The diastereomeric ratio (71:29) was determined by ${ }^{1} \mathrm{H}$ NMR of the crude product mixture.

${ }^{1} \mathrm{H}$ NMR (400 MHz, $\mathrm{CDCl}_{3}$, mixture of diastereomers) $\delta 3.60-3.54(\mathrm{~m}, 0.5 \mathrm{H}), 3.50-3.44(\mathrm{~m}, 0.5 \mathrm{H})$, $3.43-3.35(\mathrm{~m}, 1.5 \mathrm{H}), 3.29-3.22(\mathrm{~m}, 1 \mathrm{H}), 3.21-3.13(\mathrm{~m}, 1 \mathrm{H}), 3.12-3.09(\mathrm{~m}, 1 \mathrm{H}), 3.02(\mathrm{~s}, 3 \mathrm{H}), 3.00$ $-2.97(\mathrm{~m}, 0.5 \mathrm{H}), 2.94(\mathrm{~s}, 1.5 \mathrm{H}), 2.80(\mathrm{ddd}, J=13.7,11.4,4.9 \mathrm{~Hz}, 1 \mathrm{H}), 2.49-2.36(\mathrm{~m}, 1.5 \mathrm{H}), 2.25-$ $2.18(\mathrm{~m}, 1 \mathrm{H}), 2.05-1.90(\mathrm{~m}, 3 \mathrm{H}), 1.30(\mathrm{tdd}, J=13.3,11.1,3.4 \mathrm{~Hz}, 1 \mathrm{H}) .{ }^{13} \mathrm{C}\left\{{ }^{1} \mathrm{H}\right\} \mathrm{NMR}(150 \mathrm{MHz}$, $\mathrm{CDCl}_{3}$, mixture of diastereomers) $\delta 57.0,53.4,48.7,47.7,42.7,42.1,29.6,25.6,19.9,17.5,16.1,12.1$. MS (EI): $m / z=273\left(4, \mathrm{M}^{+}\right), 146$ (13), 141 (14), 132 (86), 63 (25). IR (ATR): $v=3441,3170,2929$, 2324, 1710, 1441, 1290, 1127, 1014, 940, 771. HRMS $\mathrm{m} / z$ : calcd for $\left[\mathrm{C}_{6} \mathrm{H}_{12} \mathrm{INOS}+\mathrm{H}\right]^{+}: 273.9757$, found: 273.9757 .

\section{3-(1-Iodoethyl)-1-phenyl-3,4,5,6-tetrahydro-1,2-thiazine-1-oxide $(2 \mathrm{j})$ :}

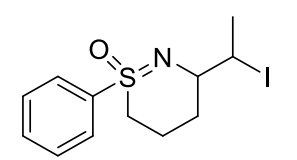

Following the general procedure but performing the reaction at $50{ }^{\circ} \mathrm{C}$ with $1 \mathbf{j}$ as starting material, provided the corresponding cyclized product $\mathbf{2 j}$ (12 $\mathrm{mg}, 11 \%$ yield) as a brown oil and as single diastereomer.

${ }^{1} \mathrm{H}$ NMR $\left(600 \mathrm{MHz}, \mathrm{CDCl}_{3}\right) \delta 8.18(\mathrm{~d}, J=8.3 \mathrm{~Hz}, 2 \mathrm{H}), 7.64(\mathrm{t}, J=7.4 \mathrm{~Hz}, 1 \mathrm{H})$, $7.56(\mathrm{t}, J=7.8 \mathrm{~Hz}, 2 \mathrm{H}), 4.46(\mathrm{qd}, J=7.1,3.9 \mathrm{~Hz}, 1 \mathrm{H}), 3.16(\mathrm{dt}, J=11.8,3.4 \mathrm{~Hz}, 1 \mathrm{H}), 2.94(\mathrm{dt}, J=$ $10.9,3.6 \mathrm{~Hz}, 1 \mathrm{H}), 2.81(\mathrm{ddd}, J=13.8,11.8,4.6 \mathrm{~Hz}, 1 \mathrm{H}), 2.58(\mathrm{qt}, J=14.1,3.3 \mathrm{~Hz}, 1 \mathrm{H}), 2.28(\mathrm{dp}, J=$ 17.6, 3.9 Hz, 1H), $2.01-1.98(\mathrm{~m}, 1 \mathrm{H}), 1.96(\mathrm{~d}, J=7.1 \mathrm{~Hz}, 3 \mathrm{H}), 1.55(\mathrm{tdd}, J=13.6,10.9,3.3 \mathrm{~Hz}, 1 \mathrm{H})$. 
${ }^{13} \mathrm{C}\left\{{ }^{1} \mathrm{H}\right\}$ NMR $\left(150 \mathrm{MHz}, \mathrm{CDCl}_{3}\right) \delta 139.2,133.4,129.2,128.9,58.3,51.1,42.3,27.2,24.8,20.1$. MS (EI): $m / z=349\left(2, \mathrm{M}^{+}\right.$), 222 (11), 194 (4), 125 (16), 109 (15), 77 (18). IR (ATR): $v=3441,2923,2325$, $2114,1705,1446,1242,1090,988,744$. HRMS $m / z$ : calcd for $\left[\mathrm{C}_{12} \mathrm{H}_{16} \mathrm{INOS}+\mathrm{H}\right]^{+}: 350.0070$, found: 350.0078

\section{3-(Iodomethyl)-1-phenyl-4,5-dihydro-3H-isothiazole-1-oxide (4a):}

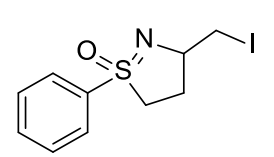

mixture. $2 \mathrm{H}), 7.68-7.61(\mathrm{~m}, 1.5 \mathrm{H}), 7.60-7.52(\mathrm{~m}, 3 \mathrm{H}), 4.34-4.26(\mathrm{~m}, 1 \mathrm{H}), 4.24-4.19(\mathrm{~m}, 0.5 \mathrm{H}), 3.67(\mathrm{ddd}$, $J=9.7,3.9,1.9 \mathrm{~Hz}, 1 \mathrm{H}), 3.59-3.51(\mathrm{~m}, 2 \mathrm{H}), 3.48-3.39(\mathrm{~m}, 1 \mathrm{H}), 3.33-3.22(\mathrm{~m}, 2 \mathrm{H}), 2.70-2.60(\mathrm{~m}$, $1.5 \mathrm{H}), 2.32-2.22(\mathrm{~m}, 1 \mathrm{H}), 2.12-2.04(\mathrm{~m}, 0.5 \mathrm{H}) .{ }^{13} \mathrm{C}\left\{{ }^{1} \mathrm{H}\right\}$ NMR $\left(150 \mathrm{MHz}, \mathrm{CDCl}_{3}\right.$, mixture of diastereomers) $\delta 139.2,138.1,133.8,133.7,129.8,129.3,129.2,129.2,69.8,66.2,57.6,57.0,31.9$, 31.4, 16.5, 13.7. MS (EI): $m / z=321\left(3, \mathrm{M}^{+}\right), 194$ (50), 180 (100), 125 (46), 77 (12). IR (ATR): $v=$ $3059,2937,2325,1723,1442,1206,1102,921,736\left(\mathrm{~cm}^{-1}\right)$. HRMS $\mathrm{m} / z$ : calcd for $\left[\mathrm{C}_{10} \mathrm{H}_{12} \mathrm{INOS}+\mathrm{H}\right]^{+}: 321.9757$, found: 321.9758 .

\section{1-(4-Bromophenyl)-3-(iodomethyl)-4,5-dihydro-3H-isothiazole-1-oxide (4b):}

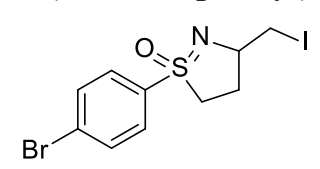

Fowing the general procedure, $\mathbf{3 b}$ provided the corresponding cyclized product 4b (98 $\mathrm{mg}, 82 \%$ yield) as a light yellow viscous oil.

product mixture.

${ }^{1} \mathrm{H}$ NMR (600 MHz, $\mathrm{CDCl}_{3}$, mixture of diastereomers) $\delta 8.01(\mathrm{~d}, J=8.7 \mathrm{~Hz}, 2 \mathrm{H}), 7.79(\mathrm{~d}, J=8.7 \mathrm{~Hz}$, $2 \mathrm{H}), 7.69(\mathrm{t}, J=8.7 \mathrm{~Hz}, 4 \mathrm{H}), 4.30-4.24(\mathrm{~m}, 1 \mathrm{H}), 4.20-4.16(\mathrm{~m}, 1 \mathrm{H}), 3.66(\mathrm{dd}, J=9.7,3.9 \mathrm{~Hz}, 1 \mathrm{H})$, $3.59-3.55(\mathrm{~m}, 3 \mathrm{H}), 3.48-3.38(\mathrm{~m}, 2 \mathrm{H}), 3.31-3.19(\mathrm{~m}, 2 \mathrm{H}), 2.68-2.60(\mathrm{~m}, 2 \mathrm{H}), 2.30-2.23(\mathrm{~m}$, $1 \mathrm{H}), 2.11-2.05(\mathrm{~m}, 1 \mathrm{H}) .{ }^{13} \mathrm{C}\left\{{ }^{1} \mathrm{H}\right\} \mathrm{NMR}\left(150 \mathrm{MHz}, \mathrm{CDCl}_{3}\right.$, mixture of diastereomers $) \delta 137.2,132.6$, 132.6, 132.5, 131.4, 130.7, 129.4, 129.2, 66.0, 57.6, 57.1, 32.0, 31.4, 16.8, 13.4. MS (EI): $\mathrm{m} / z=399(2$, $\mathrm{M}^{+}$), 272 (26), 260 (99), 258 (100), 203 (55), 187(3), 155 (11). IR (ATR): $v=3074,2939,2314,1727$, $1569,1465,1208,1100,820,739$. HRMS $m / z$ : calcd for $\left[\mathrm{C}_{10} \mathrm{H}_{11} \mathrm{BrINOS}+\mathrm{H}\right]^{+}: 399.8862$, found: 399.8862 .

\section{1-(4-Chlorophenyl)-3-(iodomethyl)-4,5-dihydro-3H-isothiazole-1-oxide (4c):}

product $4 \mathbf{c}(90 \mathrm{mg}, 84 \%$ yield $)$ as a light yellow viscous oil.
The diastereomeric ratio $(73: 27)$ was determined by ${ }^{1} \mathrm{H}$ NMR of the crude product mixture.

${ }^{1} \mathrm{H} \mathrm{NMR}\left(600 \mathrm{MHz}, \mathrm{CDCl}_{3}\right.$, mixture of diastereomers) $\delta 8.04(\mathrm{~d}, J=8.7 \mathrm{~Hz}, 0.9 \mathrm{H}), 7.83(\mathrm{~d}, J=8.6 \mathrm{~Hz}$, $2 \mathrm{H}), 7.52-7.45(\mathrm{~m}, 2.8 \mathrm{H}), 4.25-4.19(\mathrm{~m}, 1 \mathrm{H}), 4.16-4.10(\mathrm{~m}, 0.4 \mathrm{H}), 3.63-3.59(\mathrm{~m}, 1 \mathrm{H}), 3.56-$ $3.49(\mathrm{~m}, 1.9 \mathrm{H}), 3.42(\mathrm{t}, J=8.0 \mathrm{~Hz}, 1 \mathrm{H}), 3.27-3.18(\mathrm{~m}, 2 \mathrm{H}), 2.64-2.55(\mathrm{~m}, 1.4 \mathrm{H}), 2.24-2.16(\mathrm{~m}$, $1 \mathrm{H}), 2.07-2.02(\mathrm{~m}, 0.4 \mathrm{H}) .{ }^{13} \mathrm{C}\left\{{ }^{1} \mathrm{H}\right\}$ NMR $\left(150 \mathrm{MHz}, \mathrm{CDCl}_{3}\right.$, mixture of diastereomers) $\delta 140.7,140.5$, $137.8,136.7,131.3,130.6,129.6,129.5,69.7,66.0,57.6,57.2,32.0,31.4,16.8,13.6$. MS (EI): $m / z=$ 
$355\left(3, \mathrm{M}^{+}\right), 228(33), 214(100), 159(40), 111$ (6). IR (ATR): $v=3076,2940,2324,1737,1573,1208$, $1088,824,755$. HRMS $m / z$ : calcd for $\left[\mathrm{C}_{10} \mathrm{H}_{11} \mathrm{ClINOS}+\mathrm{H}\right]^{+}: 355.9367$, found: 355.9365 .

\section{3-(Iodomethyl)-1-( $p$-tolyl)-4,5-dihydro-3H-isothiazole-1-oxide (4d):}

Following the general procedure, 3d provided the corresponding cyclized
product $4 \mathbf{d}(89 \mathrm{mg}, 89 \%$ yield) as a light yellow viscous oil.
The diastereomeric ratio (79:21) was determined by ${ }^{1} \mathrm{H}$ NMR of the crude product mixture.

${ }^{1} \mathrm{H}$ NMR $\left(600 \mathrm{MHz}, \mathrm{CDCl}_{3}\right.$, mixture of diastereomers) $\delta 8.00(\mathrm{dd}, J=8.5,2.2 \mathrm{~Hz}, 2 \mathrm{H}), 7.81(\mathrm{~d}, J=6.4$ $\mathrm{Hz}, 0.7 \mathrm{H}), 7.39-7.32(\mathrm{~m}, 2.7 \mathrm{H}), 4.32-4.26(\mathrm{~m}, 0.3 \mathrm{H}), 4.22-4.17(\mathrm{~m}, 1 \mathrm{H}), 3.68(\mathrm{ddd}, J=7.1,3.8$, $1.9 \mathrm{~Hz}, 0.3 \mathrm{H}), 3.60-3.55(\mathrm{~m}, 1 \mathrm{H}), 3.53-3.49(\mathrm{~m}, 1.3 \mathrm{H}), 3.45-3.39(\mathrm{~m}, 2 \mathrm{H}), 3.28-3.22(\mathrm{~m}, 0.6 \mathrm{H})$, $2.68-2.61(\mathrm{~m}, 1.3 \mathrm{H}), 2.45(\mathrm{~s}, 3.9 \mathrm{H}), 2.29-2.22(\mathrm{~m}, 0.3 \mathrm{H}), 2.13-2.03(\mathrm{~m}, 1 \mathrm{H}) .{ }^{13} \mathrm{C}\left\{{ }^{1} \mathrm{H}\right\}$ NMR $(150$ $\mathrm{MHz}, \mathrm{CDCl}_{3}$, mixture of diastereomers) $\delta 144.9,134.9,130.0,129.9,129.8,129.2,128.7,128.3,69.6$, 66.1, 57.7, 57.1, 34.3, 31.9, 31.4, 21.6, 16.6, 13.8. MS (EI): $\mathrm{m} / \mathrm{z}=335$ (1, $\left.\mathrm{M}^{+}\right), 208$ (38), 194 (100), 139 (40), 91 (9). IR (ATR): $v=2939,2318,1735,1425,1205,1101,920,716$. HRMS $m / z$ : calcd for $\left[\mathrm{C}_{11} \mathrm{H}_{14} \mathrm{INOS}+\mathrm{H}\right]^{+}: 335.9914$, found: 335.9911 .

\section{3-(Iodomethyl)-1-(4-methoxyphenyl)-4,5-dihydro-3H-isothiazole-1-oxide (4e):}

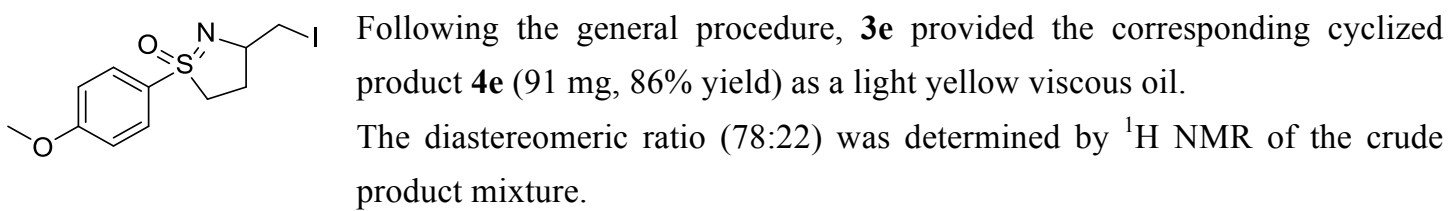

${ }^{1} \mathrm{H}$ NMR $\left(400 \mathrm{MHz}, \mathrm{CDCl}_{3}\right.$, mixture of diastereomers) $\delta 8.04(\mathrm{~d}, J=8.9 \mathrm{~Hz}, 2 \mathrm{H}), 7.85(\mathrm{~d}, J=8.9 \mathrm{~Hz}$, $1 \mathrm{H}), 7.06-6.96(\mathrm{~m}, 3 \mathrm{H}), 4.32-4.25(\mathrm{~m}, 0.5 \mathrm{H}), 4.19-4.14(\mathrm{~m}, 1 \mathrm{H}), 3.87(\mathrm{~s}, 4.5 \mathrm{H}), 3.65(\mathrm{dd}, J=9.7$, $3.8 \mathrm{~Hz}, 0.5 \mathrm{H}), 3.59-3.49(\mathrm{~m}, 2.5 \mathrm{H}), 3.45-3.37(\mathrm{~m}, 2 \mathrm{H}), 3.28-3.22(\mathrm{~m}, 0.5 \mathrm{H}), 2.69-2.57(\mathrm{~m}, 1.5 \mathrm{H})$, $2.28-2.24(\mathrm{~m}, 0.5 \mathrm{H}), 2.12-2.03(\mathrm{~m}, 1 \mathrm{H}),{ }^{13} \mathrm{C}\left\{{ }^{1} \mathrm{H}\right\} \mathrm{NMR}\left(150 \mathrm{MHz}, \mathrm{CDCl}_{3}\right.$, mixture of diastereomers) $\delta 164.2,132.1,131.6,128.5,114.8,114.6,68.6,65.5,57.7,57.1,55.8,55.8,31.8,29.7$, 16.6, 13.4. MS (EI): $m / z=351\left(4, \mathrm{M}^{+}\right), 224$ (16), 210 (46), 155 (100), 139 (8), 107 (5). IR (ATR): $v=$ $3433, \quad 2937, \quad 2302, \quad 1737, \quad 1491,1209, \quad 1104,1021, \quad 828$. HRMS $m / z$ : calcd for $\left[\mathrm{C}_{11} \mathrm{H}_{14} \mathrm{INO}_{2} \mathrm{~S}+\mathrm{H}\right]^{+}: 351.9863$, found: 351.9850 .

\section{3-(Iodomethyl)-1-(naphthalen-2-yl)-4,5-dihydro-3H-isothiazole-1-oxide (4f):}

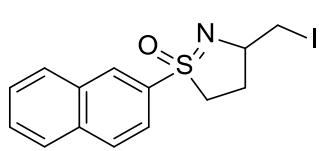

Following the general, 3f provided providing the corresponding cyclized product $\mathbf{4 f}$ ( $79 \mathrm{mg}, 71 \%$ yield) as a light yellow viscous oil.

The diastereomeric ratio (80:20) was determined by ${ }^{1} \mathrm{H}$ NMR of the crude product mixture.

${ }^{1} \mathrm{H}$ NMR (600 MHz, $\mathrm{CDCl}_{3}$, mixture of diastereomers) $\delta 8.18(\mathrm{~s}, 1 \mathrm{H}), 8.00-7.88(\mathrm{~m}, 4 \mathrm{H}), 7.63-7.53$ (m, 4H), $3.81-3.77(\mathrm{~m}, 1 \mathrm{H}), 3.67-3.62(\mathrm{~m}, 0.3 \mathrm{H}), 3.31-3.27(\mathrm{~m}, 1.6 \mathrm{H}), 3.27-3.25(\mathrm{~m}, 0.3 \mathrm{H}), 3.24$ $-3.22(\mathrm{~m}, 1 \mathrm{H}), 3.20-3.13(\mathrm{~m}, 1 \mathrm{H}), 3.02-2.94(\mathrm{~m}, 1.3 \mathrm{H}), 2.20-2.13(\mathrm{~m}, 1 \mathrm{H}), 2.09-2.02(\mathrm{~m}, 0.3 \mathrm{H})$, $1.99-1.93(\mathrm{~m}, 0.3 \mathrm{H}), 1.92-1.84(\mathrm{~m}, 1 \mathrm{H}) .{ }^{13} \mathrm{C}\left\{{ }^{1} \mathrm{H}\right\}$ NMR (150 MHz, $\mathrm{CDCl}_{3}$, mixture of diastereomers) $\delta$ 139.6, 134.4, 132.8, 129.6, 128.5, 128.5, 128.1, 127.9, 127.9, 127.5, 127.4, 125.1, 124.9, 119.7, 69.7, 69.4, 52.6, 52.5, 29.7, 29.1, 24.7, 13.8. MS (EI): $m / z=371\left(2, \mathrm{M}^{+}\right), 244$ (6), 230 (5), 175 (37), 127 (65). IR (ATR): $v=3323,2919,2320,1722,1407,1264$, 996, 744. HRMS $m / z$ : calcd for $\left[\mathrm{C}_{14} \mathrm{H}_{14} \mathrm{INOS}+\mathrm{H}\right]^{+}: 371.9914$, found: 371.9922 . 


\section{1-Benzyl-3-(iodomethyl)-4,5-dihydro-3H-116-isothiazole-1-oxide (4g):}

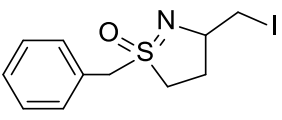

Following the general procedure, $\mathbf{3 g}$ provided the corresponding cyclized product $4 \mathrm{~g}$ (69 $\mathrm{mg}, 69 \%$ yield) as a light yellow viscous oil.

The diastereomeric ratio (71:29) was determined by ${ }^{1} \mathrm{H}$ NMR of the crude product mixture.

${ }^{1} \mathrm{H}$ NMR $\left(600 \mathrm{MHz}, \mathrm{CDCl}_{3}\right.$, mixture of diastereomers) $\delta 7.41(\mathrm{~s}, 7 \mathrm{H}), 4.26(\mathrm{~s}, 2.8 \mathrm{H}), 4.02-3.87(\mathrm{~m}$, $1 \mathrm{H}), 3.66-3.41(\mathrm{~m}, 2 \mathrm{H}), 3.17-3.05(\mathrm{~m}, 0.8 \mathrm{H}), 3.01-2.92(\mathrm{~m}, 2 \mathrm{H}), 2.80(\mathrm{dd}, J=4.8,3.9 \mathrm{~Hz}, 0.4 \mathrm{H})$, $2.51(\mathrm{dd}, J=4.8,2.6 \mathrm{~Hz}, 0.4 \mathrm{H}), 2.35(\mathrm{dd}, J=5.4,0.9 \mathrm{~Hz}, 0.4 \mathrm{H}), 2.26-2.13(\mathrm{~m}, 1 \mathrm{H}), 2.04-1.87(\mathrm{~m}$, $1 \mathrm{H}), 1.89-1.81(\mathrm{~m}, 0.4 \mathrm{H}) .{ }^{13} \mathrm{C}\left\{{ }^{1} \mathrm{H}\right\} \mathrm{NMR}\left(150 \mathrm{MHz}, \mathrm{CDCl}_{3}\right.$, mixture of diastereomers) $\delta 130.6,130.5$, 129.2, 129.2, 128.4, 127.8, 69.4, 60.0, 50.1, 49.5, 47.5, 47.5, 47.2, 29.7, 26.4, 24.9. MS (EI): $m / z=335$ (2, $\left.\mathrm{M}^{+}\right), 208$ (2), 194 (3), 139 (2), 123 (3), 91 (100). IR (ATR): $v=3470,2931,2316,1725,1432,1279$, $1112,915,730$. HRMS $m / z$ : calcd for $\left[\mathrm{C}_{11} \mathrm{H}_{14} \mathrm{INOS}+\mathrm{H}\right]^{+}: 335.9914$, found: 335.9914 .

\subsection{Characterization Data of 5 and 6}

\section{1-Phenyl-3-((phenylthio)methyl)-3,4,5,6-tetrahydro-1, 2-thiazine-1-oxide (5):}

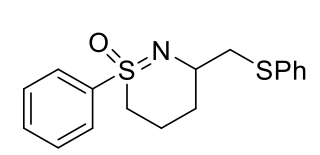

Following the synthetic procedure, iodo substrate 2 a provided the product 5 (156 mg, $98 \%$ yield) as a pink solid. mp: $79.6-81.4{ }^{\circ} \mathrm{C}$.

${ }^{1} \mathrm{H}$ NMR (600 MHz, $\mathrm{CDCl}_{3}$, mixture of diastereomers) $\delta 8.06(\mathrm{~d}, J=7.1 \mathrm{~Hz}$, 2H), $7.98(\mathrm{~d}, J=7.2 \mathrm{~Hz}, 0.7 \mathrm{H}), 7.64-7.59(\mathrm{~m}, 1 \mathrm{H}), 7.53(\mathrm{td}, J=7.7,3.7 \mathrm{~Hz}, 2.7 \mathrm{H}), 7.39(\mathrm{~d}, J=7.3$ $\mathrm{Hz}, 2.4 \mathrm{H}), 7.28-7.27(\mathrm{~m}, 02.4 \mathrm{H}), 7.25-7.24(\mathrm{~m}, 0.7 \mathrm{H}), 7.18-7.13(\mathrm{~m}, 1.7 \mathrm{H}), 3.93-3.88(\mathrm{~m}, 1 \mathrm{H})$, $3.81-3.77(\mathrm{~m}, 0.4 \mathrm{H}), 3.64-3.51(\mathrm{~m}, 0.7 \mathrm{H}), 3.33-3.27(\mathrm{~m}, 1.4 \mathrm{H}), 3.18-3.06(\mathrm{~m}, 2.7 \mathrm{H}), 2.84-2.77$ (m, 1H), $2.56-2.51(\mathrm{~m}, 1.3 \mathrm{H}), 2.29-2.25(\mathrm{~m}, 1 \mathrm{H}), 2.17-2.13(\mathrm{~m}, 1 \mathrm{H}), 2.05-1.94(\mathrm{~m}, 0.7 \mathrm{H}), 1.54-$ $1.46(\mathrm{~m}, 1 \mathrm{H}) .{ }^{13} \mathrm{C}\left\{{ }^{1} \mathrm{H}\right\}$ NMR $\left(150 \mathrm{MHz}, \mathrm{CDCl}_{3}\right.$, mixture of diastereomers) $\delta 139.9,139.3,137.1,136.3$, 133.3, 133.2, 129.2, 129.1, 129.1, 128.9, 128.8, 128.7, 128.6, 128.2, 125.8, 125.6, 54.8, 52.8, 51.3, 51.1, 42.9, 39.7, 28.4, 24.8, 20.9, 17.3.MS (EI): $m / z=317\left(1, \mathrm{M}^{+}\right), 208$ (3), 194 (100), 125 (55), 123 (40), 109 (45), 77 (70). IR (ATR): $v=3063,2927,2324,1730,1441,1221,1117,736$. HRMS $m / z$ : calcd for $\left[\mathrm{C}_{17} \mathrm{H}_{19} \mathrm{NOS}_{2}+\mathrm{H}\right]^{+}: 318.0981$, found: 318.0979 .

\section{3-(Azidomethyl)-1-phenyl-3,4,5,6-tetrahydro-1,2-thiaz-ine-1-oxide (6):}

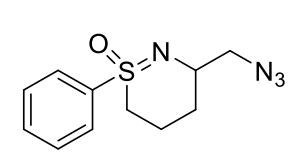

Following the synthetic procedure, iodo substrate 2 a provided the product $\mathbf{6}$ (122 $\mathrm{mg}, 97 \%$ yield) as a light brown viscous oil.

${ }^{1} \mathrm{H}$ NMR (400 MHz, $\mathrm{CDCl}_{3}$, mixture of diastereomers) $\delta 8.10-8.06(\mathrm{~m}, 2 \mathrm{H})$, $8.05-8.00(\mathrm{~m}, 0.6 \mathrm{H}), 7.68-7.59(\mathrm{~m}, 1.3 \mathrm{H}), 7.57-7.50(\mathrm{~m}, 2.6 \mathrm{H}), 3.95-3.83(\mathrm{~m}, 1 \mathrm{H}), 3.69-3.61$ (m, 0.3H), $3.57-3.54(\mathrm{~m}, 0.7 \mathrm{H}), 3.43(\mathrm{dd}, J=12.1,5.3 \mathrm{~Hz}, 1.3 \mathrm{H}), 3.27(\mathrm{dd}, J=12.1,4.6 \mathrm{~Hz}, 1 \mathrm{H})$, $3.16(\mathrm{dt}, J=11.7,3.4 \mathrm{~Hz}, 1 \mathrm{H}), 2.79(\mathrm{ddd}, J=13.8,11.7,4.7 \mathrm{~Hz}, 1 \mathrm{H}), 2.56(\mathrm{qt}, J=13.8,3.3 \mathrm{~Hz}, 1 \mathrm{H}$ ), $2.36(\mathrm{~d}, J=6.2 \mathrm{~Hz}, 0.3 \mathrm{H}), 2.29-2.27(\mathrm{~m}, 1 \mathrm{H}), 1.83-1.81(\mathrm{~m}, 1.3 \mathrm{H}), 1.68-1.53(\mathrm{~m}, 2 \mathrm{H}) .{ }^{13} \mathrm{C}\left\{{ }^{1} \mathrm{H}\right\}$ NMR (100 MHz, $\mathrm{CDCl}_{3}$, mixture of diastereomers) $\delta 133.4,133.3,129.2,129.1,128.6,128.5,58.7$, 58.3, 53.4, 53.1, 50.9, 47.4, 26.6, 24.8, 20.7, 20.5. MS (EI): $m / z=250$ (2, $\left.\mathrm{M}^{+}\right), 208$ (4), 194 (100), 125 (29), 77 (21). IR (ATR): $v=3065,2930,2326,2094,1444,1226,1125,1022,732$. HRMS $m / z$ : calcd for $\left[\mathrm{C}_{11} \mathrm{H}_{14} \mathrm{~N}_{4} \mathrm{OS}+\mathrm{Na}\right]^{+}: 273.0781$, found: 273.0782 . 


\subsection{HPLC Spectra and Characterization Data (2a and 4a)}

The diastereomers of compounds $\mathbf{2}$ and $\mathbf{4}$ could not be separated from each other by silica gel column chromatography. However, for the stereoisomers of the two representative examples $2 \mathbf{a}$ and 4a analytical CSP-HPLC conditions were developed which demonstrate that separation is feasible on a preparative level by HPLC or SFC techniques. Subsequently, the major diastereomer of 2a was separated using preparative SFC (conditions: IA column, prep: $65 \mathrm{~g} \mathrm{CO}_{2}$ with 8\% methanol, $15 \mathrm{mg} / \mathrm{mL}, 35^{\circ} \mathrm{C}$ ).

Major diastereomer of 2a: dark brown solid; mp: $68.1-68.5^{\circ} \mathrm{C} .{ }^{1} \mathrm{H}$ NMR (400 $\left.\mathrm{MHz}, \mathrm{CDCl}_{3}\right) \delta 8.23-$ $8.04(\mathrm{~m}, 2 \mathrm{H}), 7.66-7.58(\mathrm{~m}, 1 \mathrm{H}), 7.57-7.47(\mathrm{~m}, 2 \mathrm{H}), 3.49-3.34(\mathrm{~m}, 3 \mathrm{H}), 3.11(\mathrm{dt}, J=11.7,3.3 \mathrm{~Hz}$, 1H), 2.76 (ddd, $J=13.7,11.7,4.6 \mathrm{~Hz}, 1 \mathrm{H}), 2.57$ (qt, $J=13.8,3.2 \mathrm{~Hz}, 1 \mathrm{H}), 2.29-2.19$ (m, 1H), $1.98-$ $1.88(\mathrm{~m}, 1 \mathrm{H}), 1.60-1.46(\mathrm{~m}, 1 \mathrm{H}) .{ }^{13} \mathrm{C}\left\{{ }^{1} \mathrm{H}\right\}$ NMR $\left(101 \mathrm{MHz}, \mathrm{CDCl}_{3}\right) \delta 139.2,133.4,129.2,128.7$, 52.3, 50.6, 29.7, 20.4, 19.6. HRMS $m / z$ : calcd for $\left[\mathrm{C}_{11} \mathrm{H}_{14} \mathrm{INOS}+\mathrm{H}\right]^{+}: 335.9914$, found: 335.9908 .

Separation of the four stereoisomers of 2 a by analytical CSP-HPLC:

Sample Info: AD-H, Hep/iProH $=95: 5,0.8 \mathrm{~mL} / \mathrm{min}, 20^{\circ} \mathrm{C}$

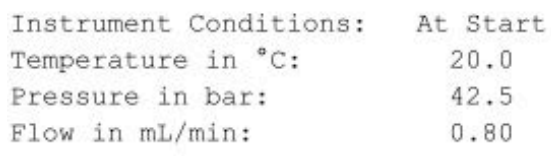

At Start
20.0
42.5
0.80

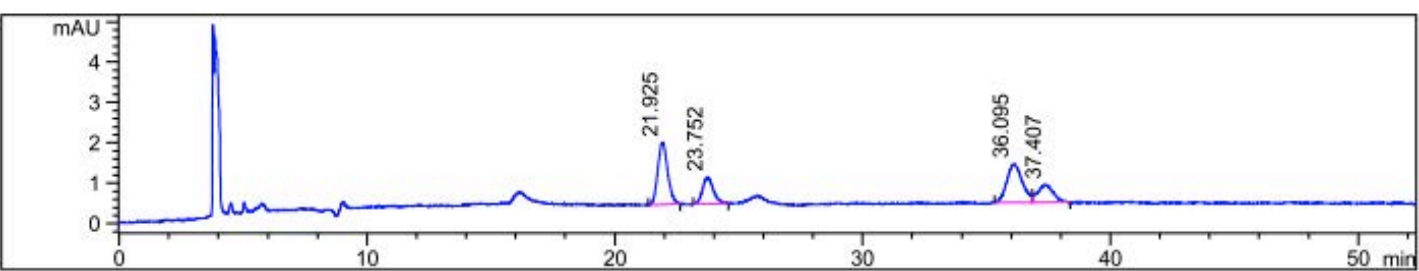

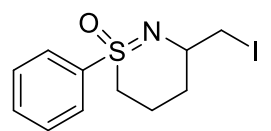

2a

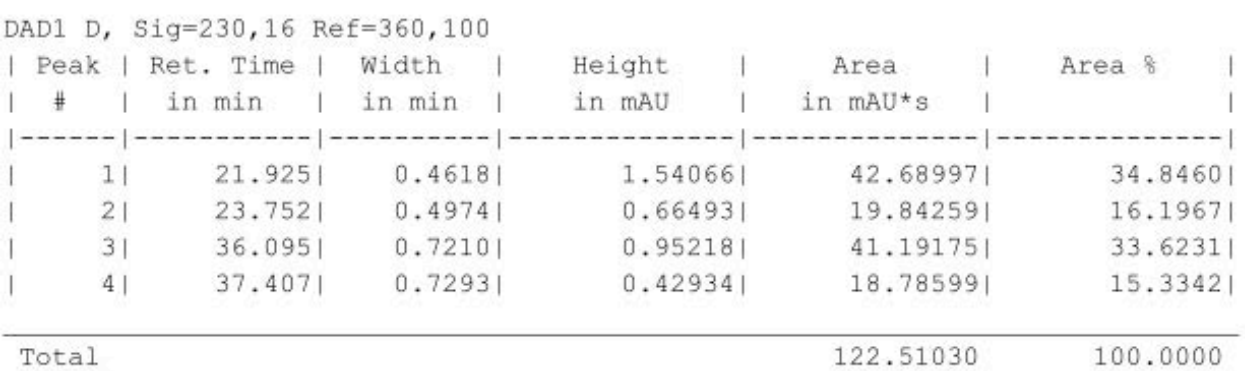


Separation of the four stereoisomers of 4 a by analytical CSP-HPLC:

Sample Info: AD-H, Hep/iPrOH $=85: 15,0.8 \mathrm{~mL} / \mathrm{min}, 20^{\circ} \mathrm{C}$

Instrument Conditions:

Temperature in ${ }^{\circ} \mathrm{C}$ :

Pressure in bar:

Flow in $\mathrm{mL} / \mathrm{min}$;

At Start
20.0
46.9
0.80

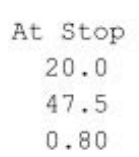

0.80

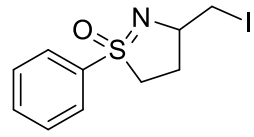

$4 a$

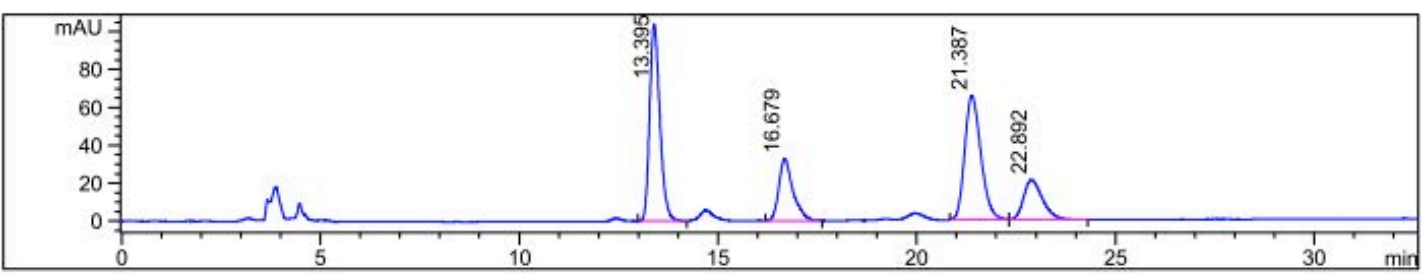

DAD1 C, Sig=210, 8 Ref $=360,100$

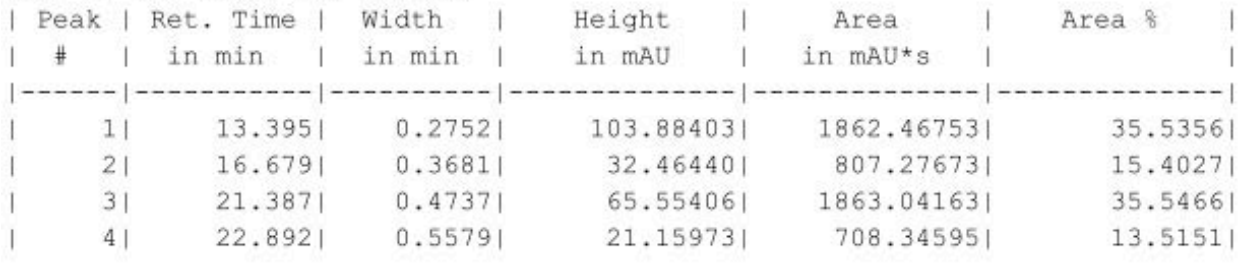

Total

5241.13184

100.0000 


\section{1. ${ }^{1} \mathrm{H}$ and ${ }^{13} \mathrm{C}$ NMR Spectra}
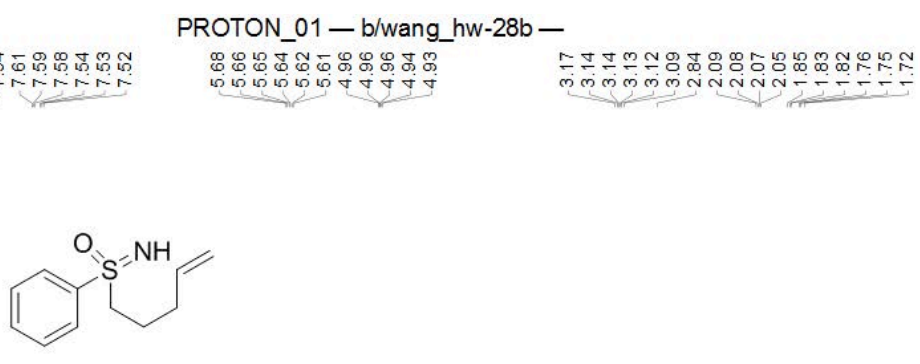

$1 \mathrm{a}$

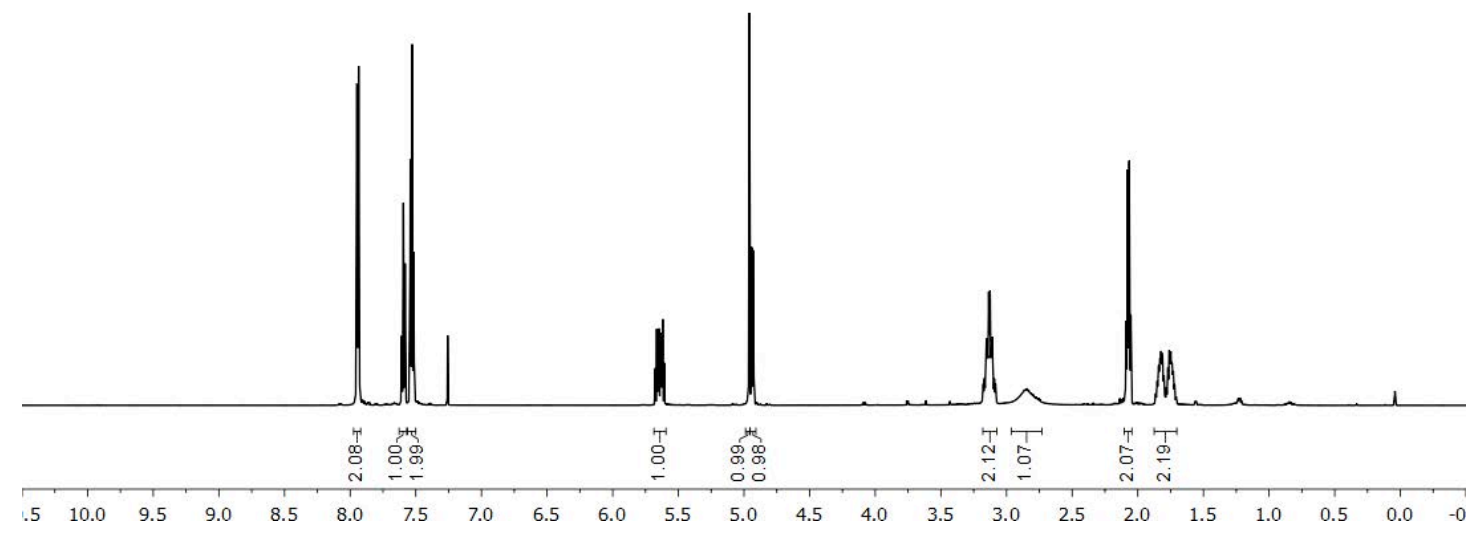

\begin{tabular}{|c|c|c|c|c|}
\hline 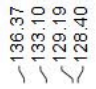 & $\frac{\substack{0 \\
\oplus}}{\stackrel{\omega}{\sigma}}$ & 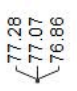 & $\begin{array}{l}m \\
\stackrel{0}{0} \\
\infty \\
1 \\
1\end{array}$ & \\
\hline
\end{tabular}

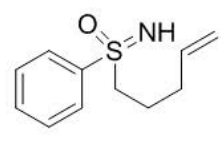

$1 \mathrm{a}$

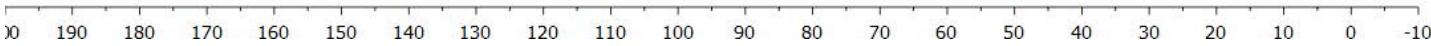




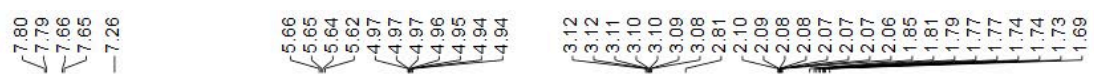

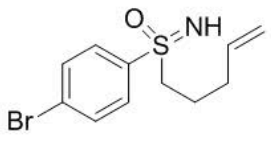

$1 \mathrm{~b}$
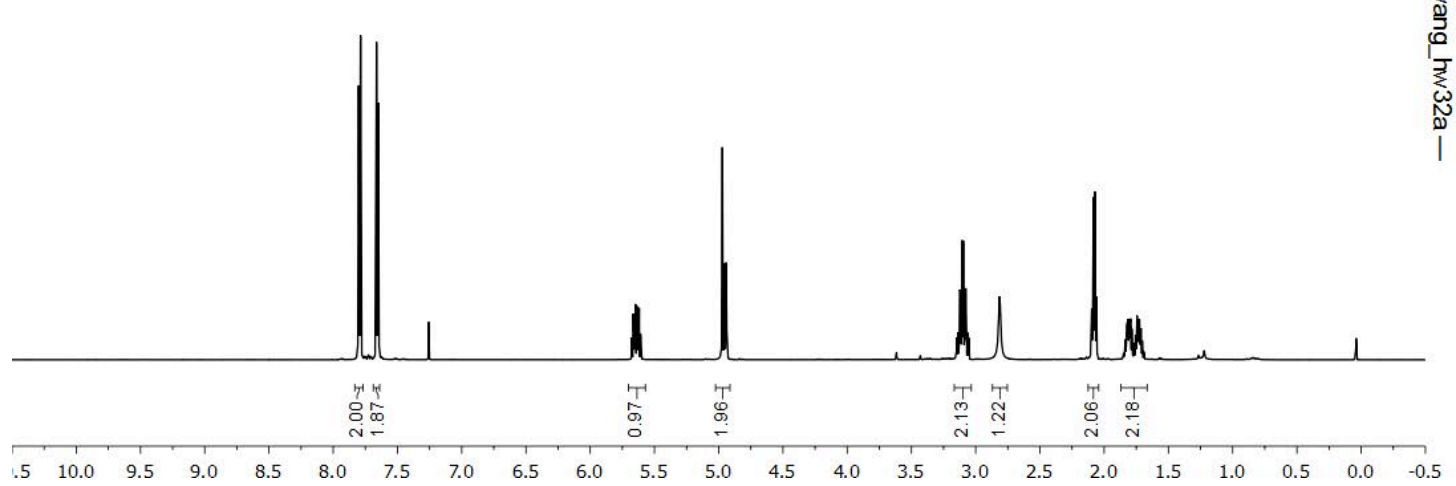

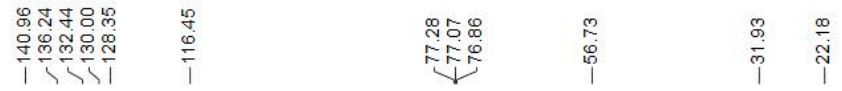<smiles>C=CCCCS(=O)(=O)c1ccc(Br)cc1</smiles>

1b

$\begin{array}{lllllllllllllllllllll}10 & 190 & 180 & 170 & 160 & 150 & 140 & 130 & 120 & 110 & 100 & 90 & 80 & 70 & 60 & 50 & 40 & 30 & 20 & 10 & 0\end{array}$ 


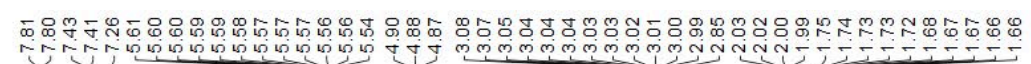

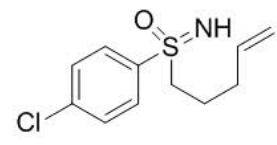

$1 \mathrm{c}$

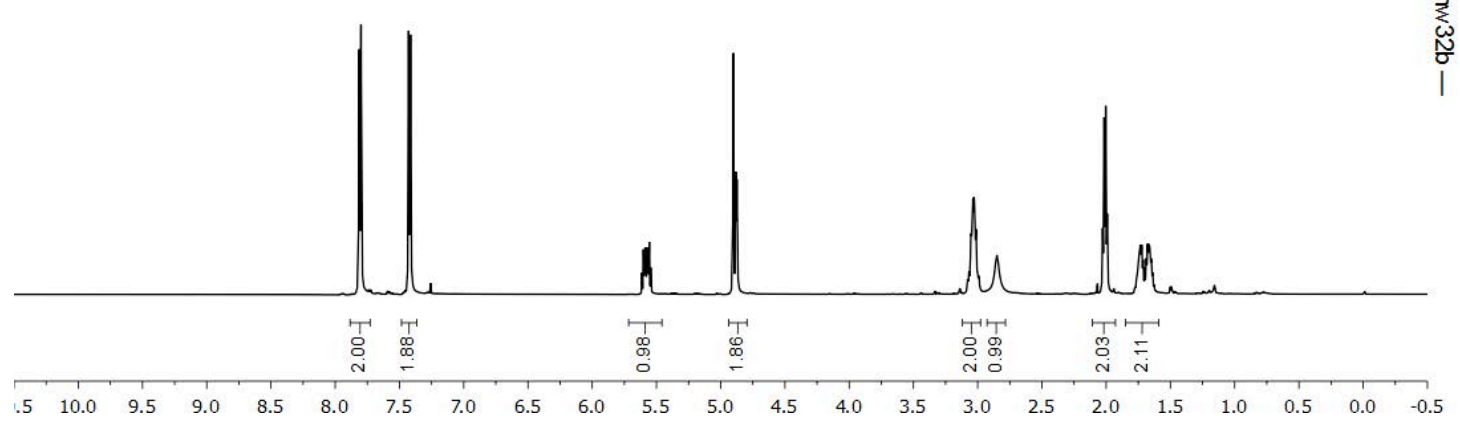

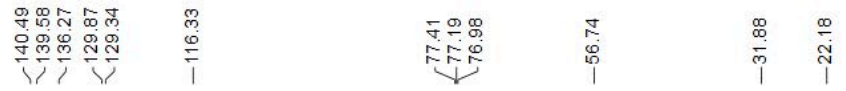
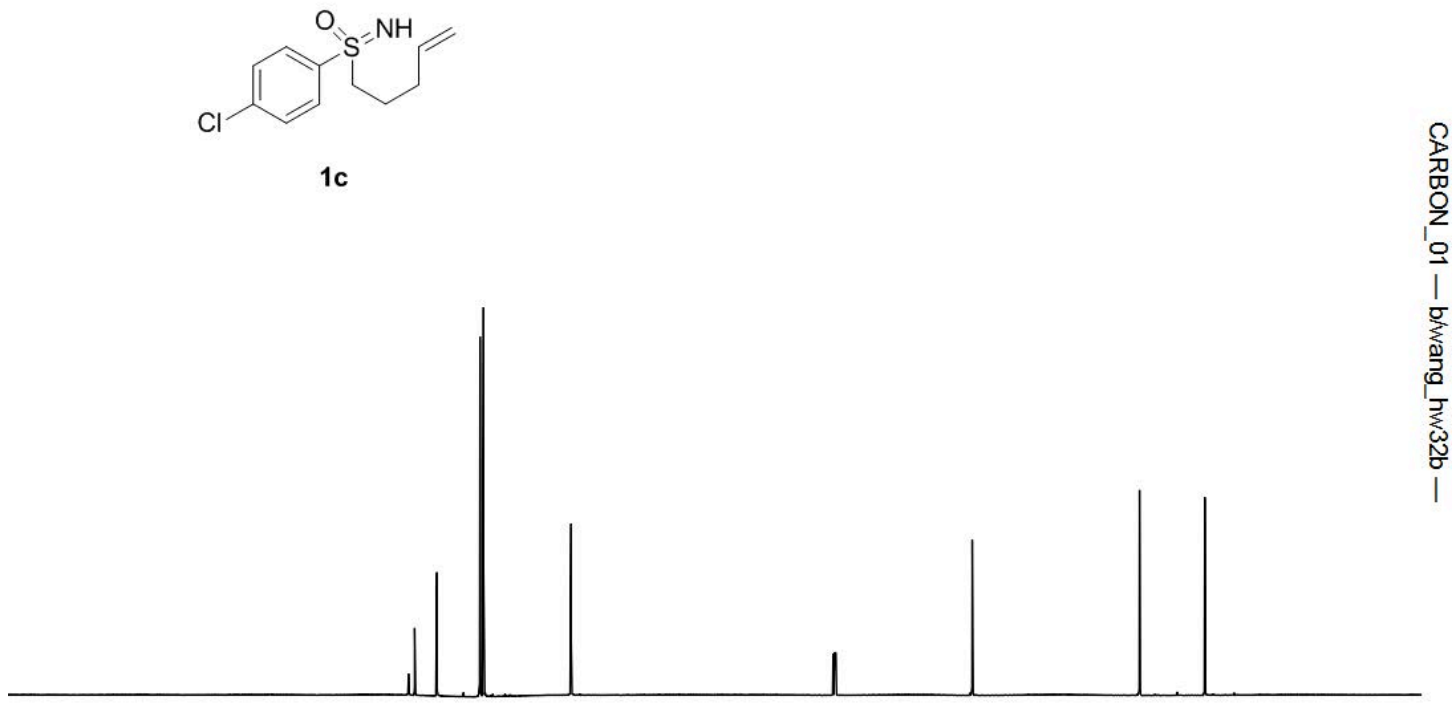

$\begin{array}{lllllllllllllllllllll}10 & 190 & 180 & 170 & 160 & 150 & 140 & 130 & 120 & 110 & 100 & 90 & 80 & 70 & 60 & 50 & 40 & 30 & 20 & 10 & 0\end{array}$ 


\section{Nom}
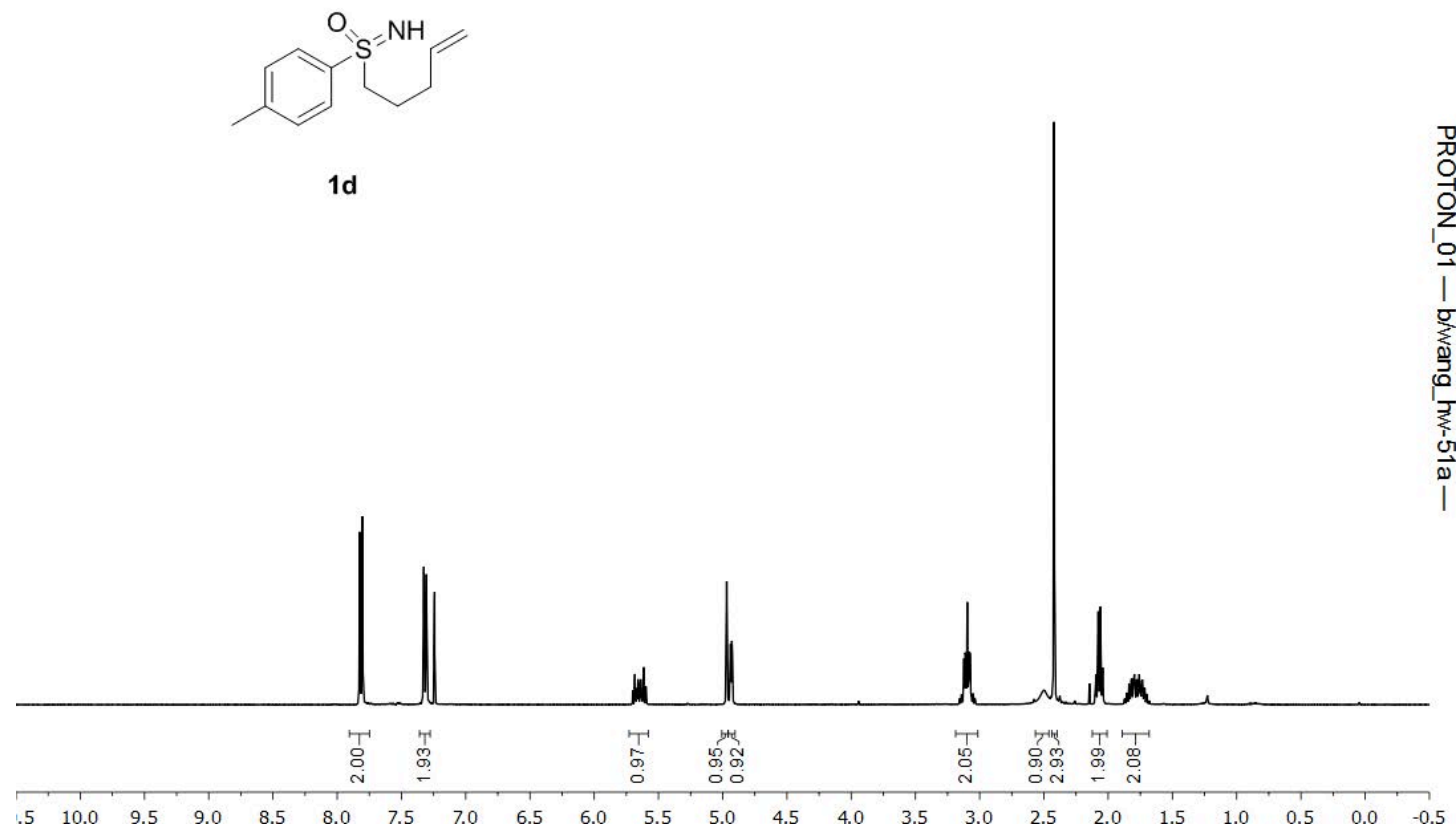

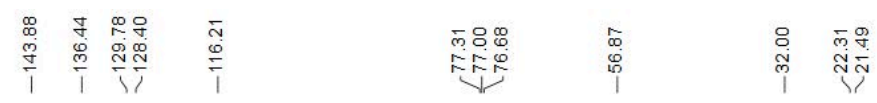

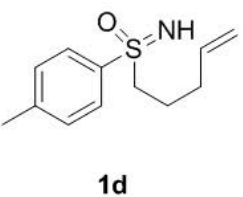

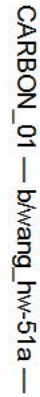

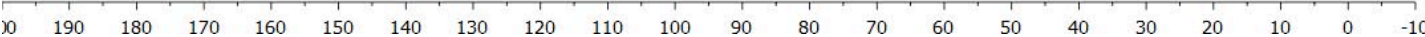




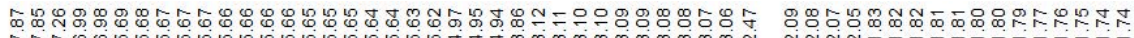

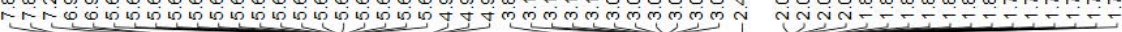

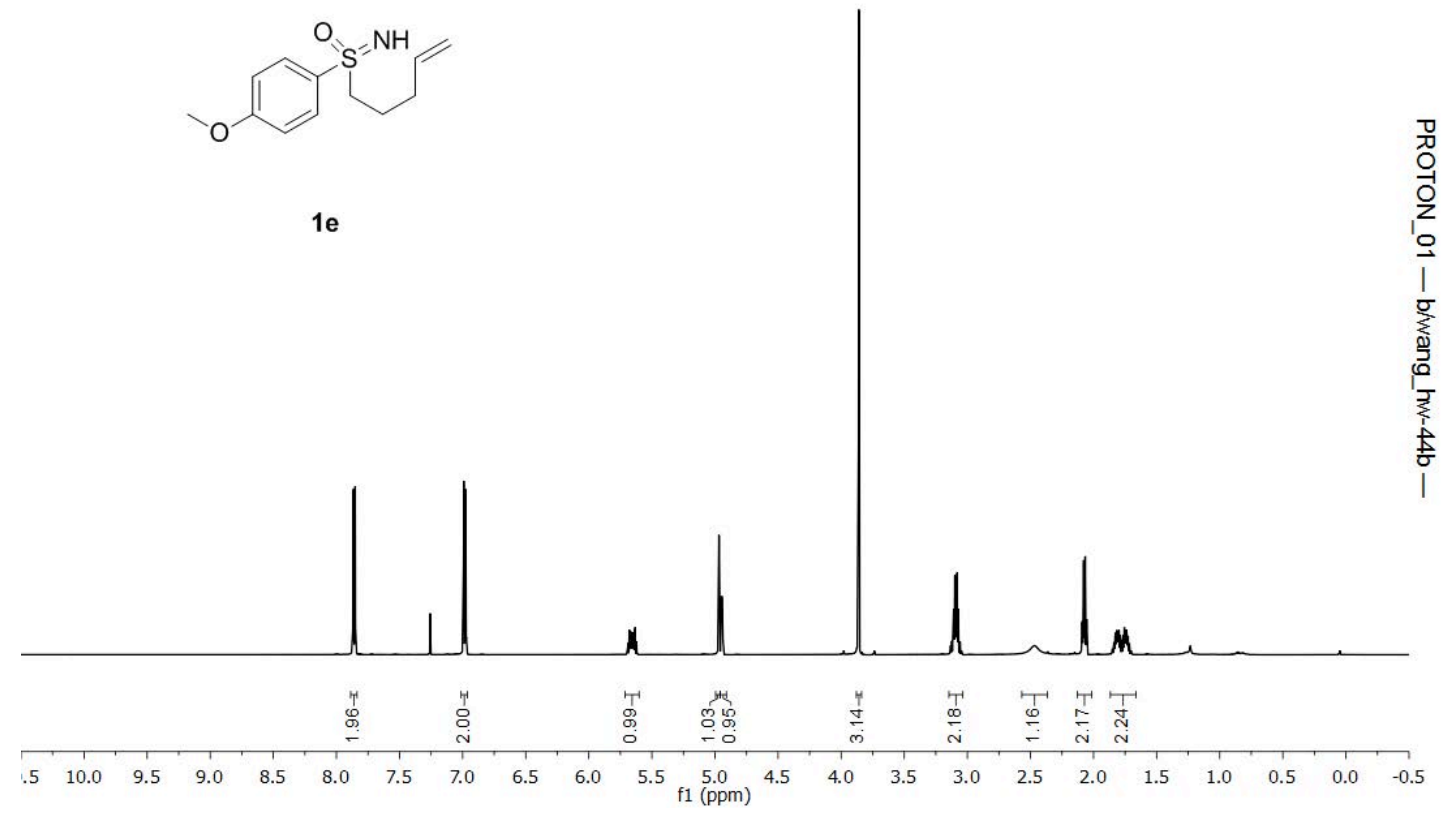

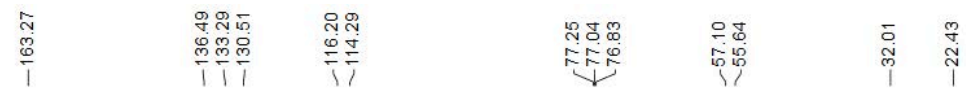

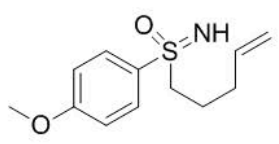

$1 \mathrm{e}$

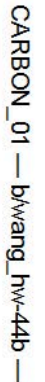

\begin{tabular}{lllllllllllllllllllllllll}
\hline 0 & 190 & 180 & 170 & 160 & 150 & 140 & 130 & 120 & 110 & 100 & 90 & 80 & 70 & 60 & 50 & 40 & 30 & 20 & 10 & 0 & -10
\end{tabular} 


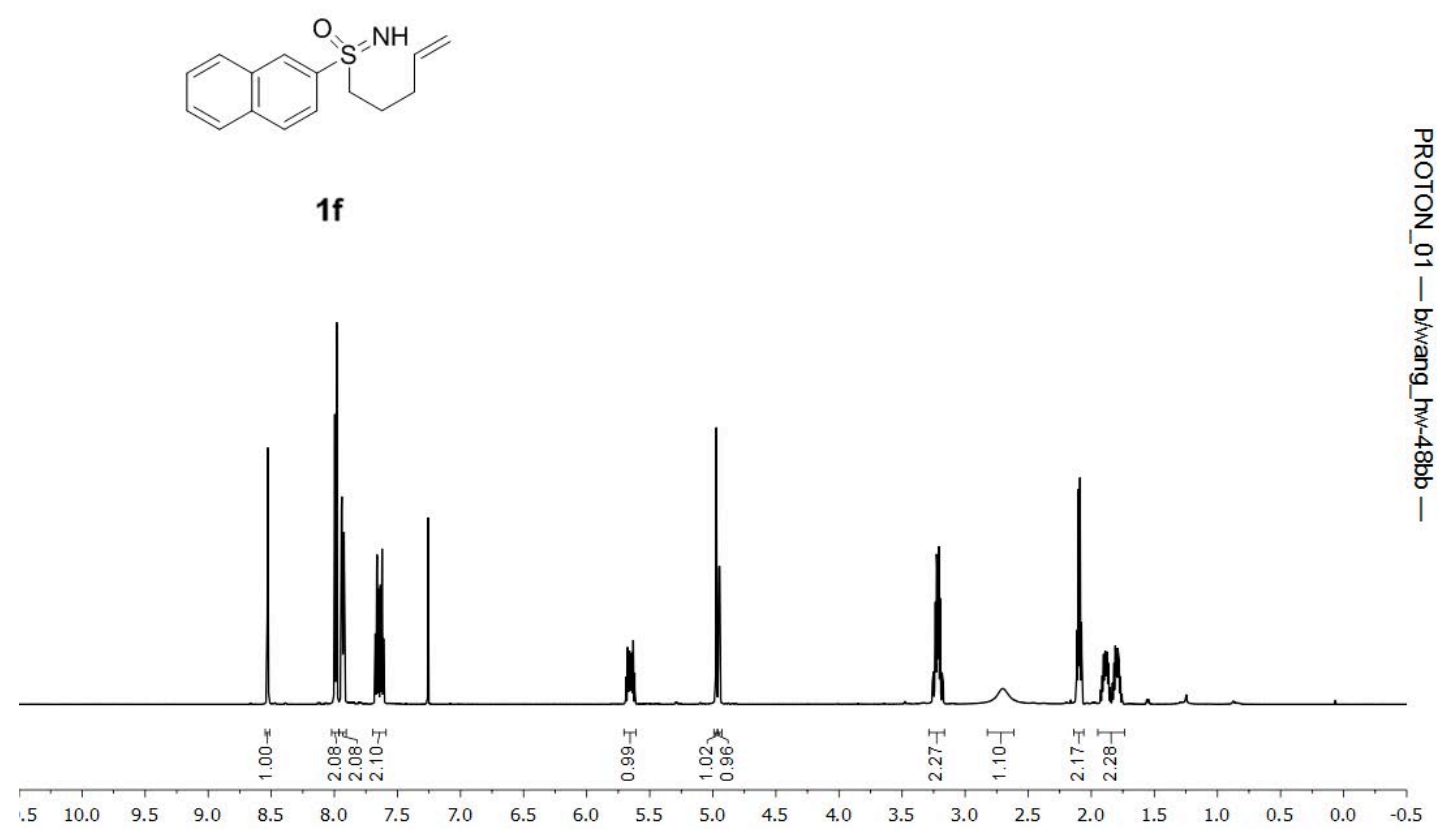

시유.

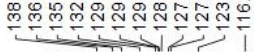

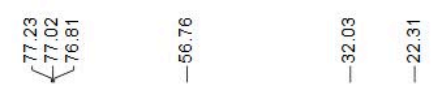

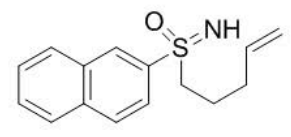

$1 f$ 

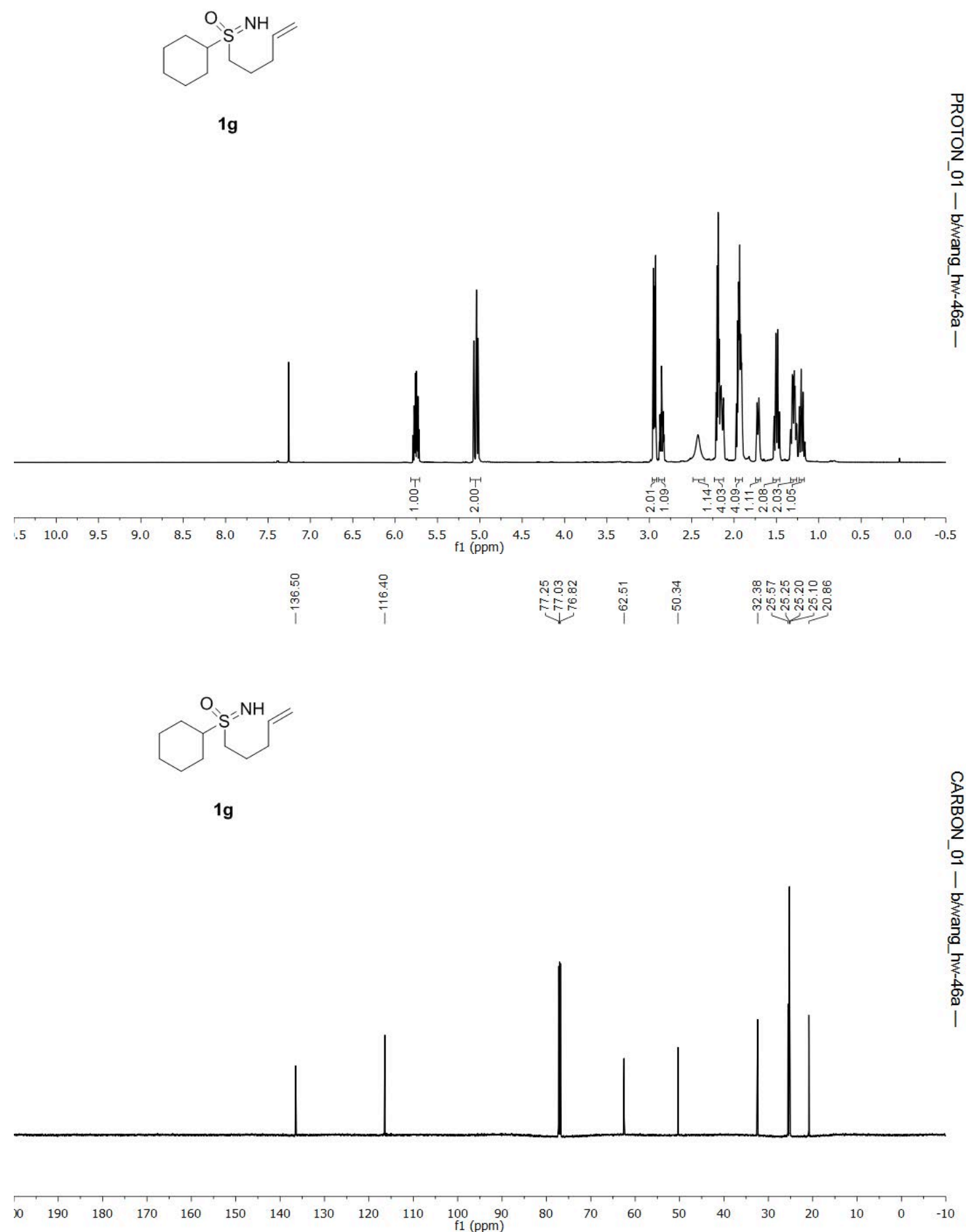


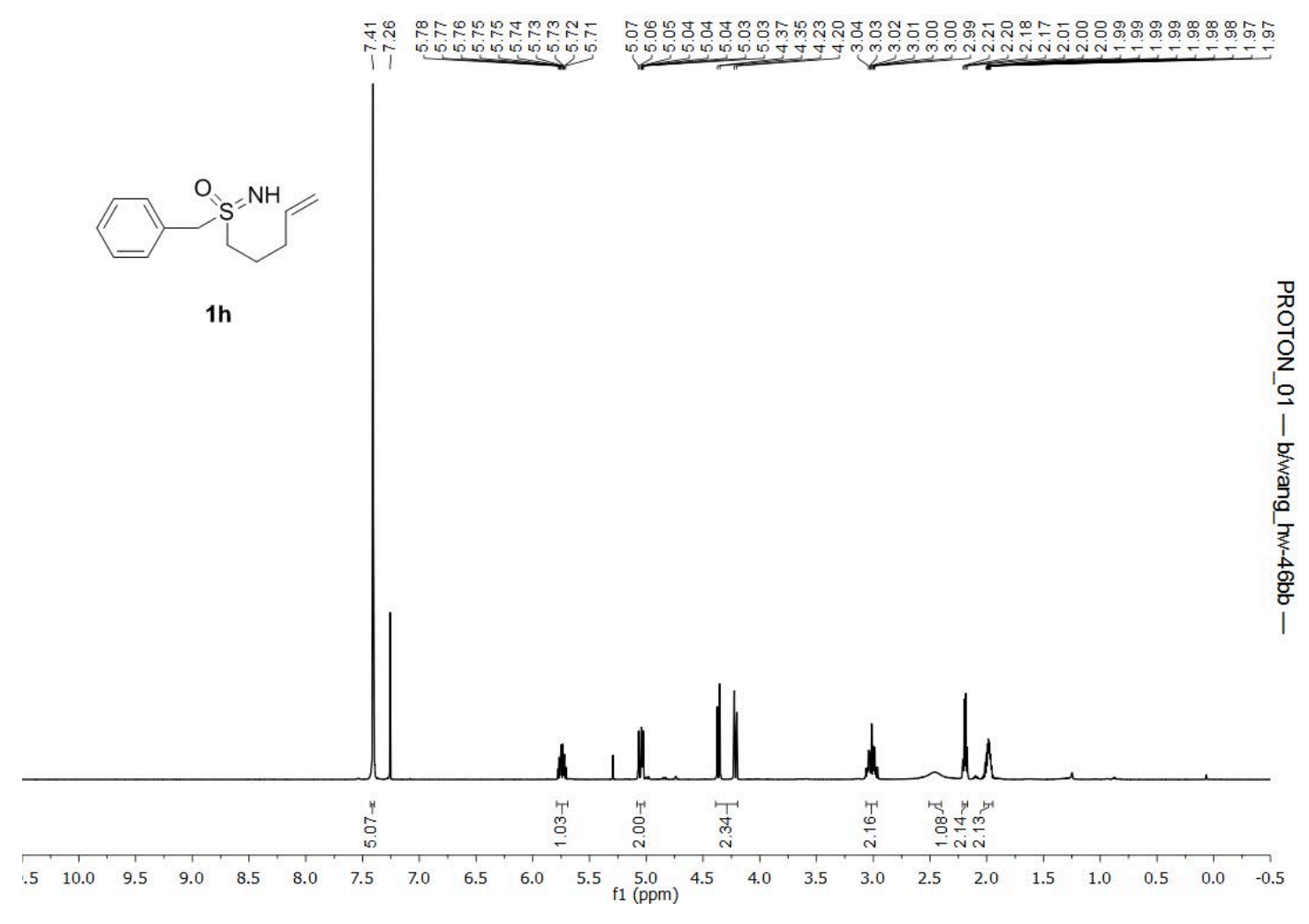

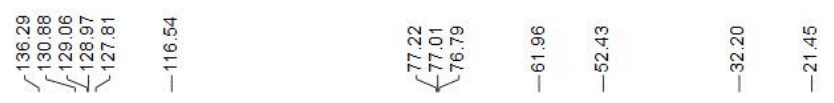

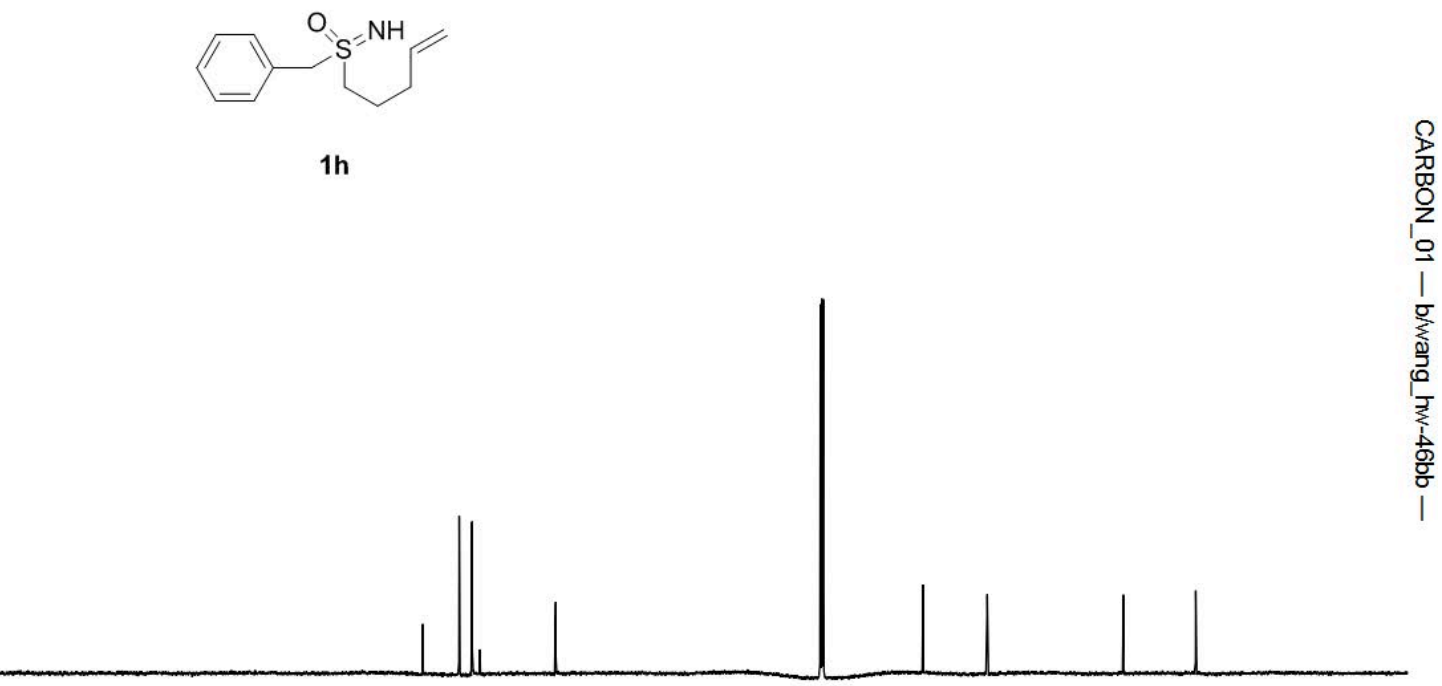

$1 \mathrm{~h}$

$\begin{array}{lllllllllllllllllllllll}10 & 190 & 180 & 170 & 160 & 150 & 140 & 130 & 120 & 110 & 100 & 90 & 80 & 70 & 60 & 50 & 40 & 30 & 20 & 10 & 0 & -10\end{array}$ 

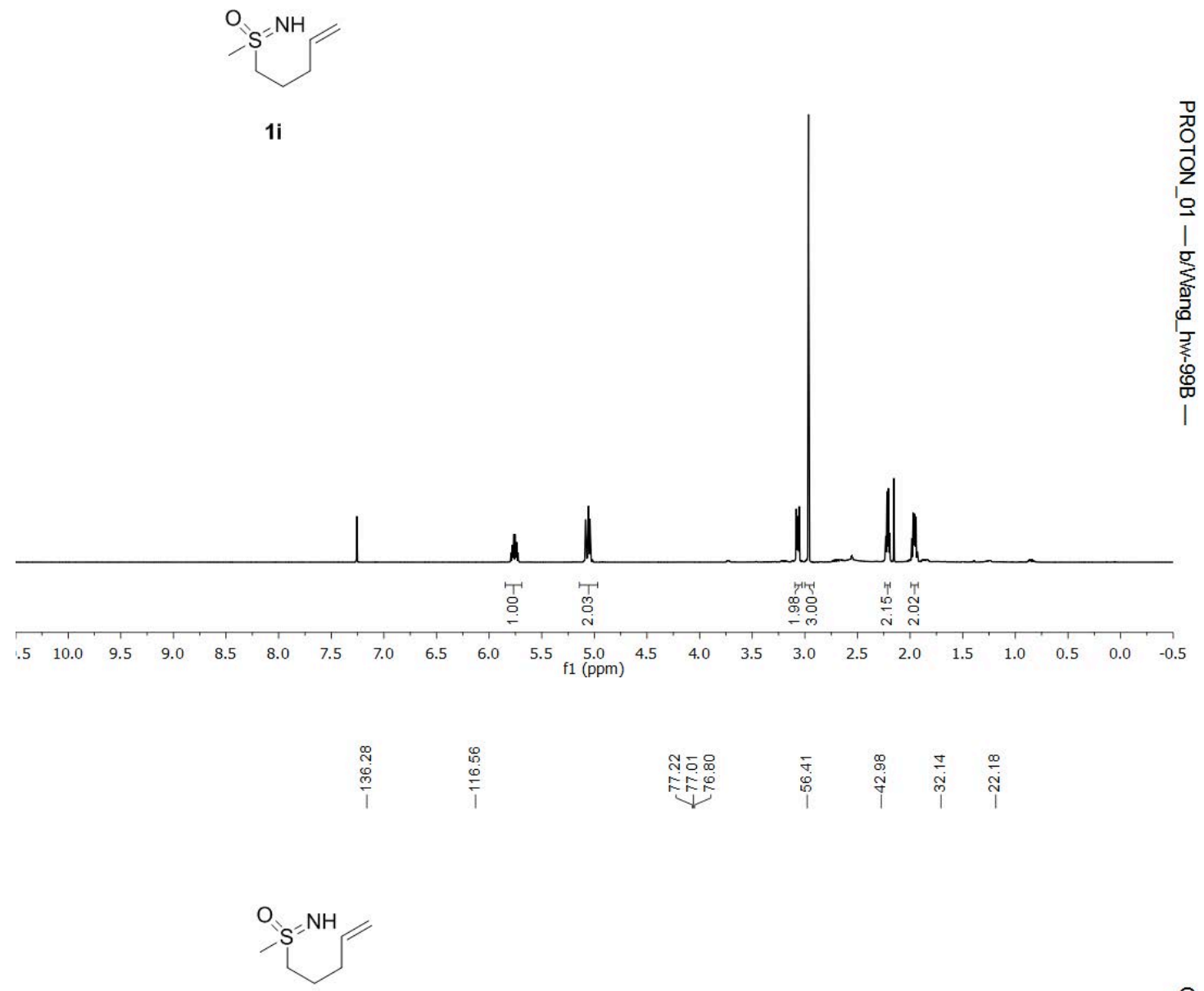

$1 i$

\begin{tabular}{lllllllllllllllllllll}
\hline 0 & 190 & 180 & 170 & 160 & 150 & 140 & 130 & 120 & 110 & $\underset{\mathrm{f} 1(\mathrm{ppm})}{100}$ & 80 & 70 & 60 & 50 & 40 & 30 & 20 & 10 & 0 & -10
\end{tabular} 


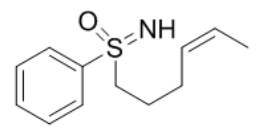

1j

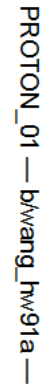

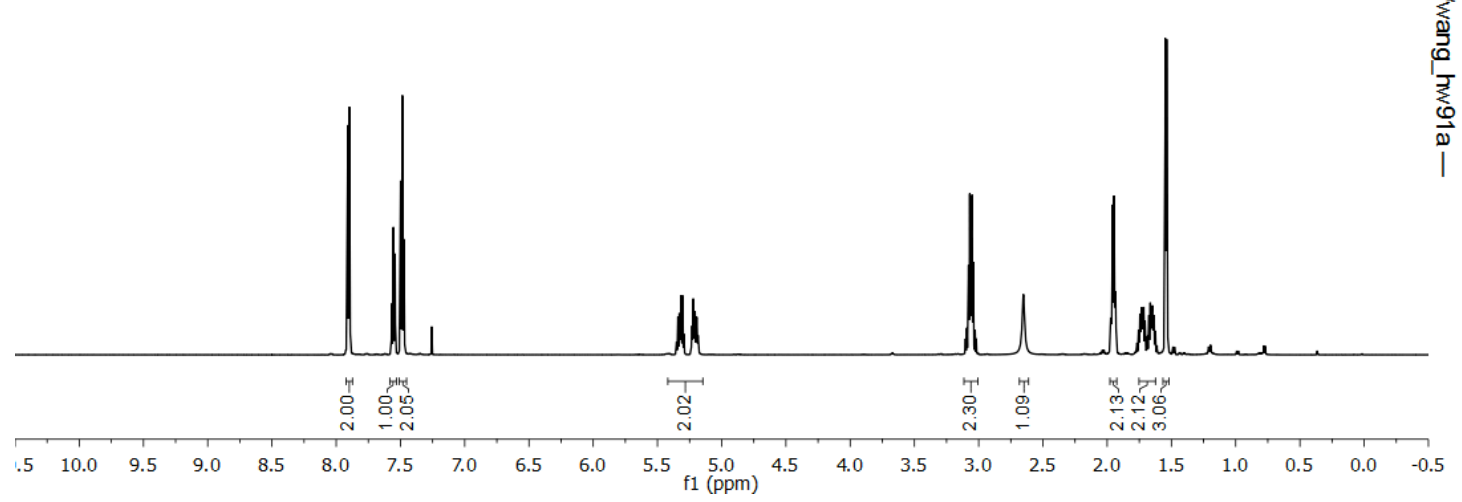

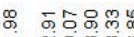

广

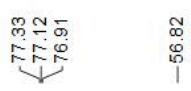

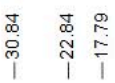

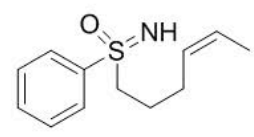

1j

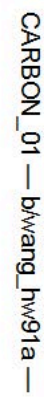

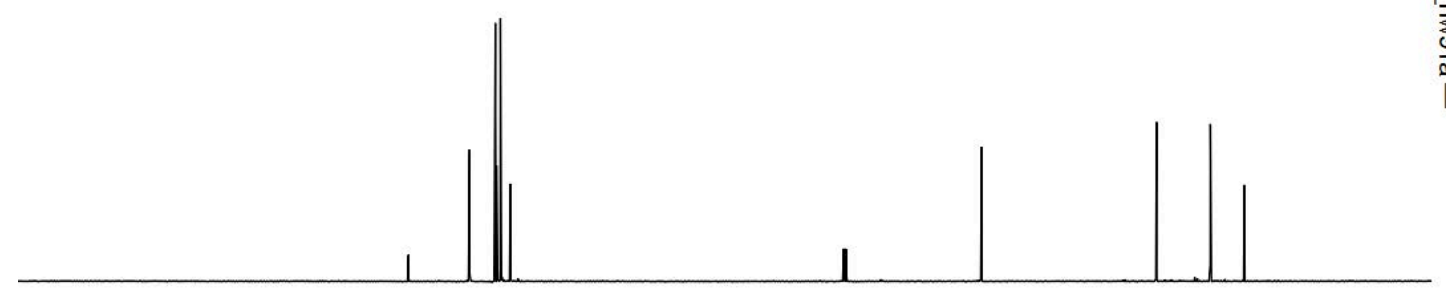

$\begin{array}{lllllllllllllllllllll}10 & 190 & 180 & 170 & 160 & 150 & 140 & 130 & 120 & 110 & \underset{f 1}{100}(\mathrm{ppm}) & 80 & 70 & 60 & 50 & 40 & 30 & 20 & 10 & 0 & -10\end{array}$ 


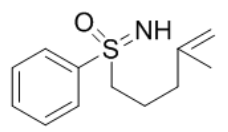

$1 \mathrm{k}$

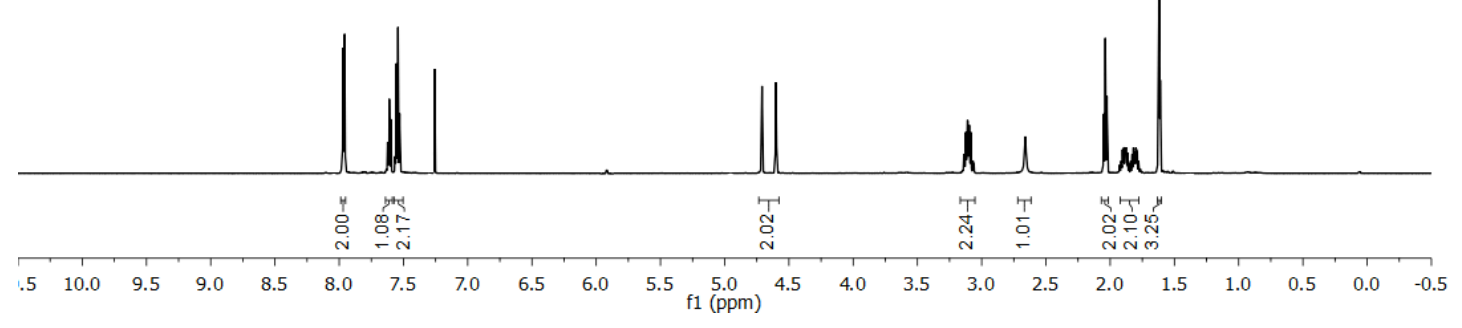

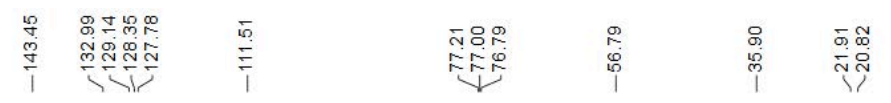

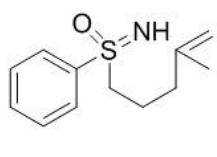

$1 \mathrm{k}$

$\begin{array}{lllllllllllllllllllllll}10 & 190 & 180 & 170 & 160 & 150 & 140 & 130 & 120 & 110 & \underset{f 1}{100}(\mathrm{ppm}) & 90 & 70 & 60 & 50 & 40 & 30 & 20 & 10 & 0 & -10\end{array}$ 

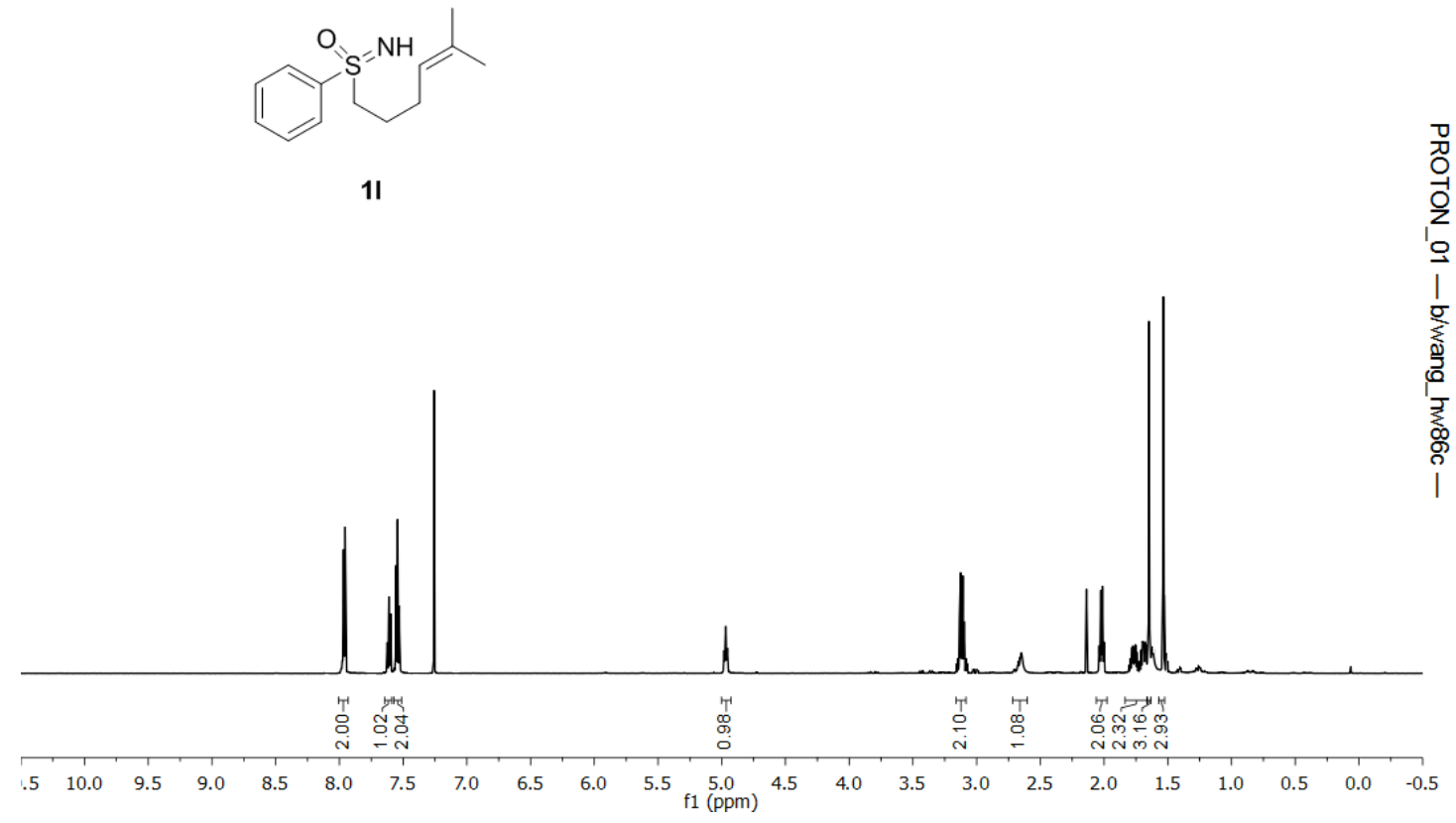

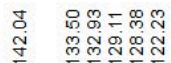

ํำ

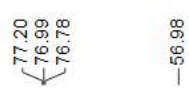

$\min$

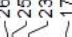

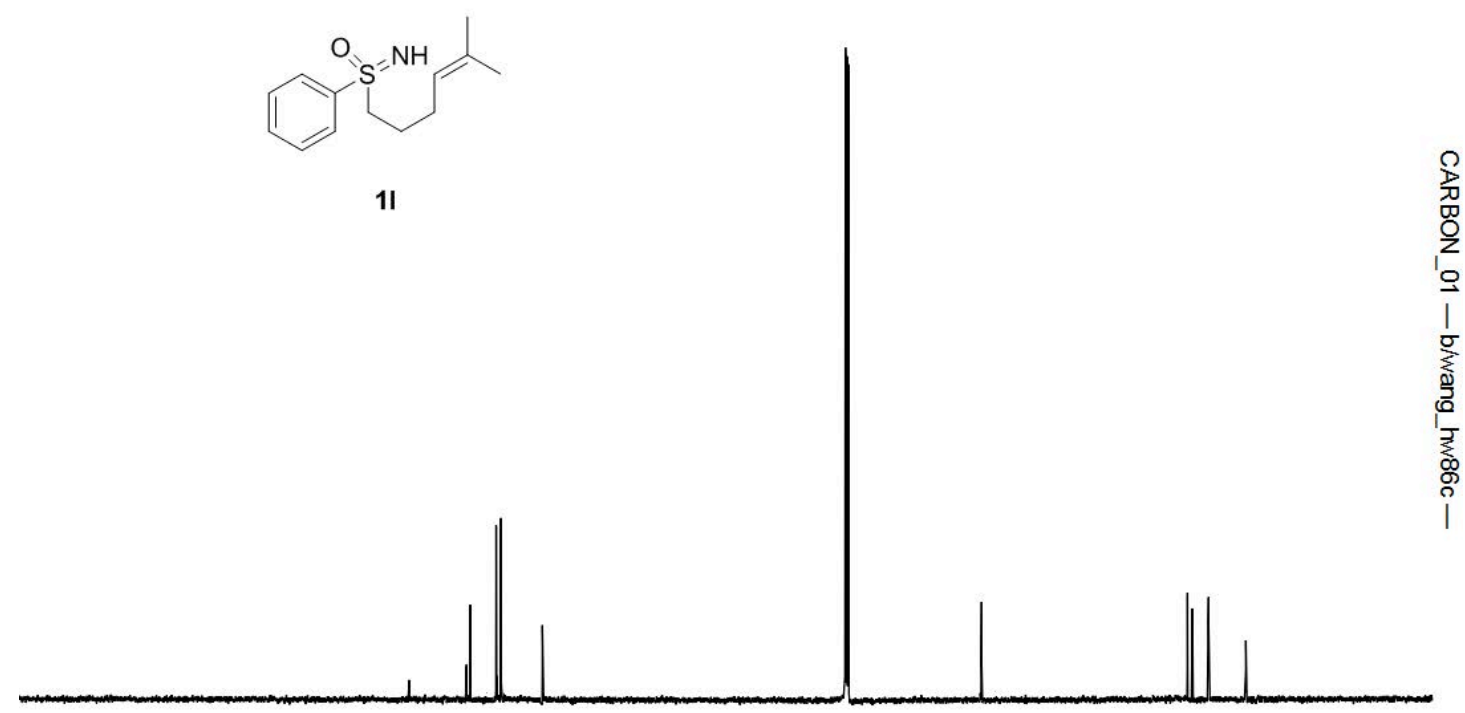

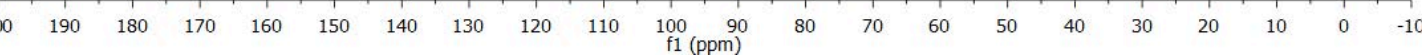




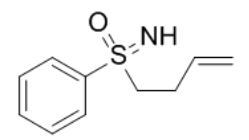

$3 a$

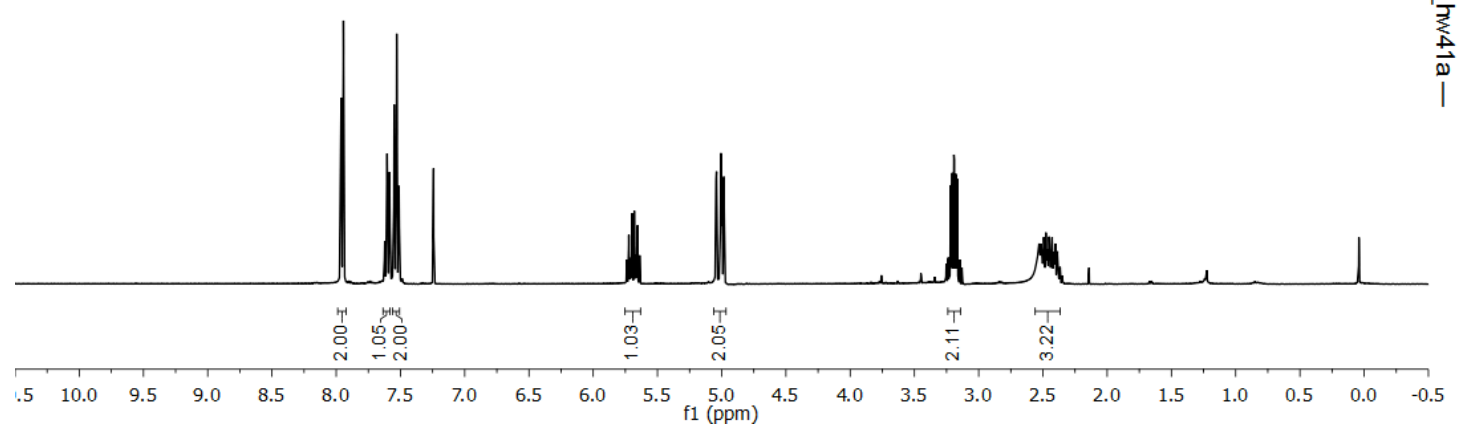

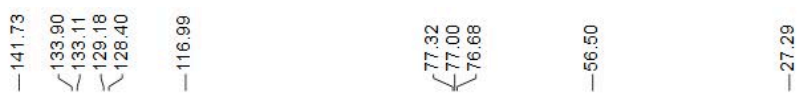

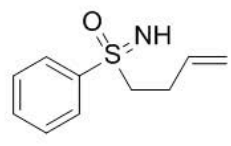

$3 a$

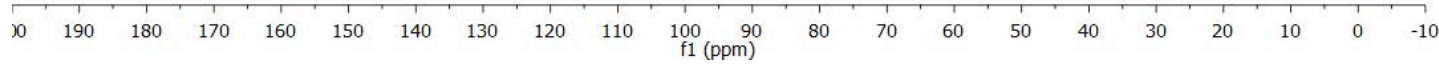


$\underbrace{-10}$

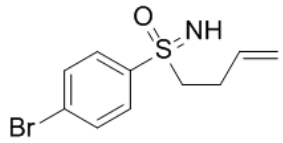

$3 \mathbf{b}$

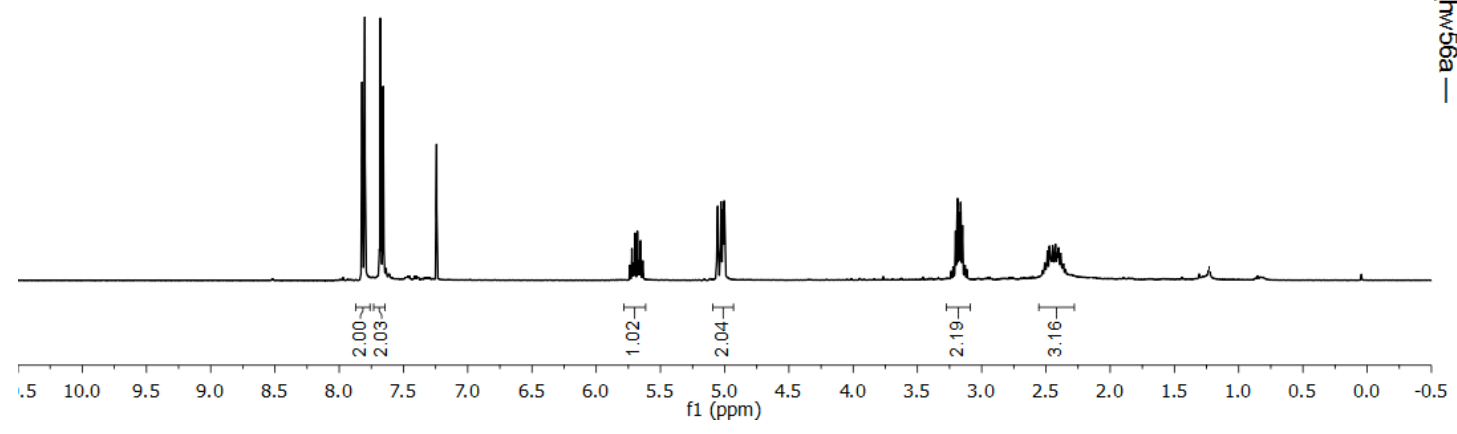

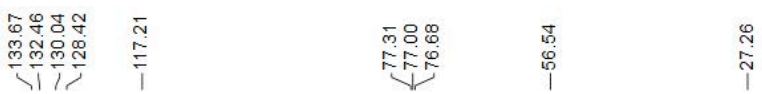

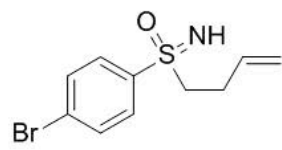

$3 \mathbf{b}$

\begin{tabular}{lllllllllllllllllllll}
\hline 0 & 190 & 180 & 170 & 160 & 150 & 140 & 130 & 120 & 110 & $\underset{\mathrm{f} 1}{100}(\mathrm{ppm})$ & 90 & 70 & 60 & 50 & 40 & 30 & 20 & 10 & 0 & -10
\end{tabular} 

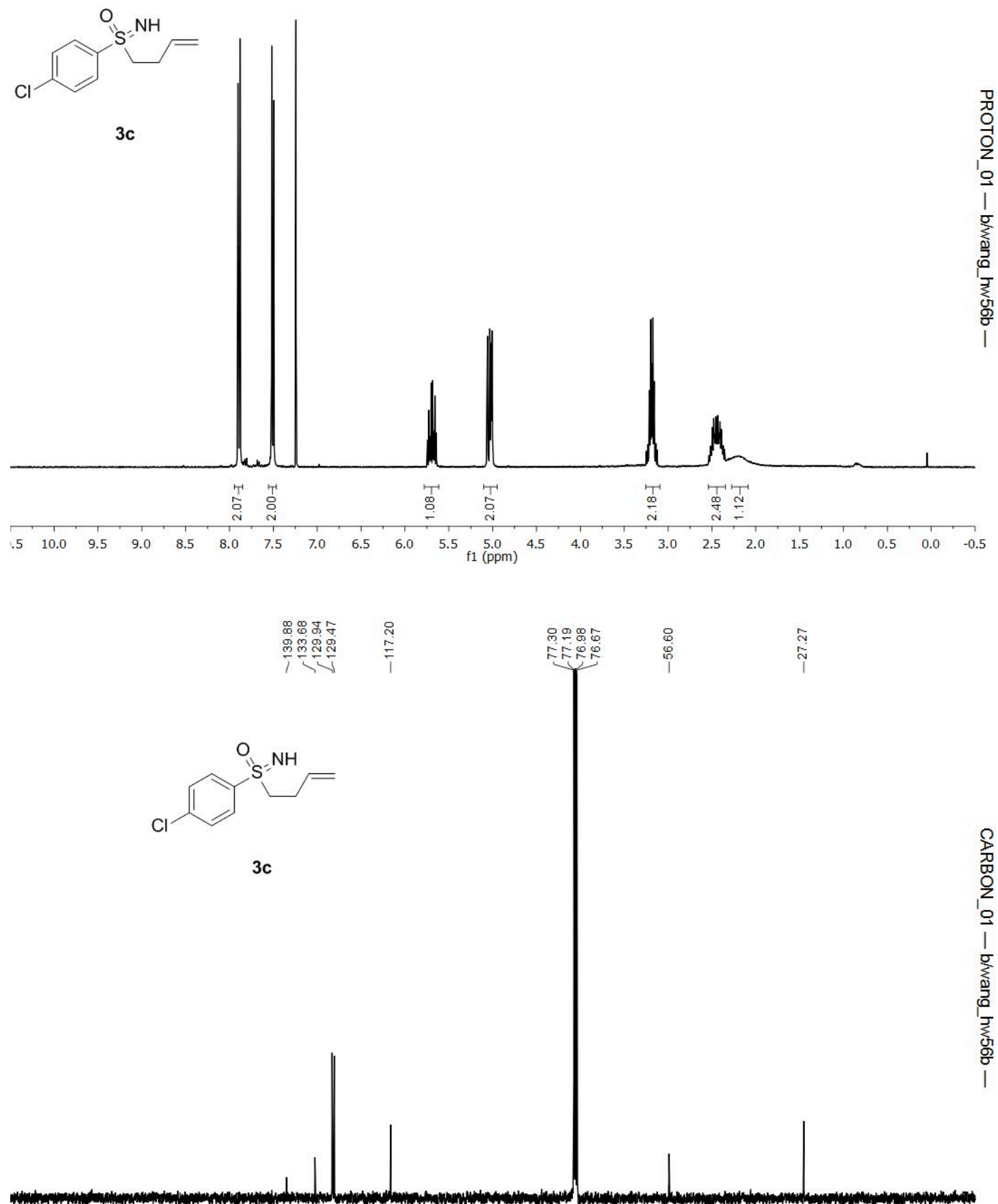

$\begin{array}{lllllllllllllllllllllllllllllll}10 & 190 & 180 & 170 & 160 & 150 & 140 & 130 & 120 & 110 & \begin{array}{l}100 \\ \mathrm{f} 1\end{array}(\mathrm{ppm}) & 90 & 80 & 70 & 60 & 50 & 40 & 30 & 20 & 10 & 0 & -10\end{array}$ 


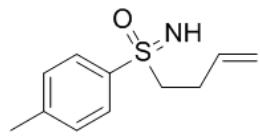

3d

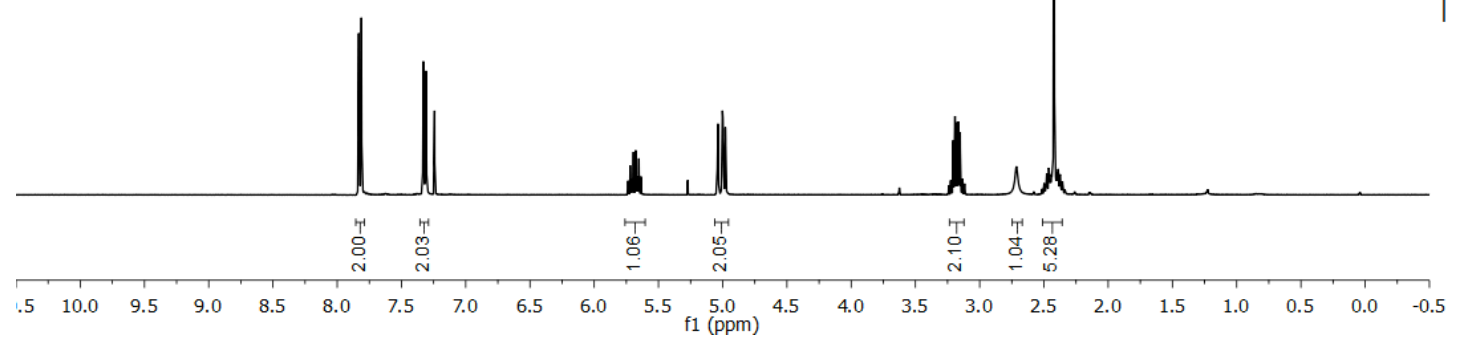

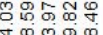

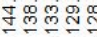
$\underset{\substack{\tilde{N} \\ \stackrel{\infty}{1}}}{1}$

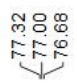
$\underset{\substack{\infty \\ \infty}}{\infty}$
$\underset{\substack{i \\ \stackrel{n}{N}}}{\substack{\text { i } \\ \text { i }}}$

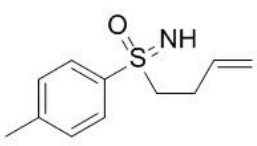

$3 d$

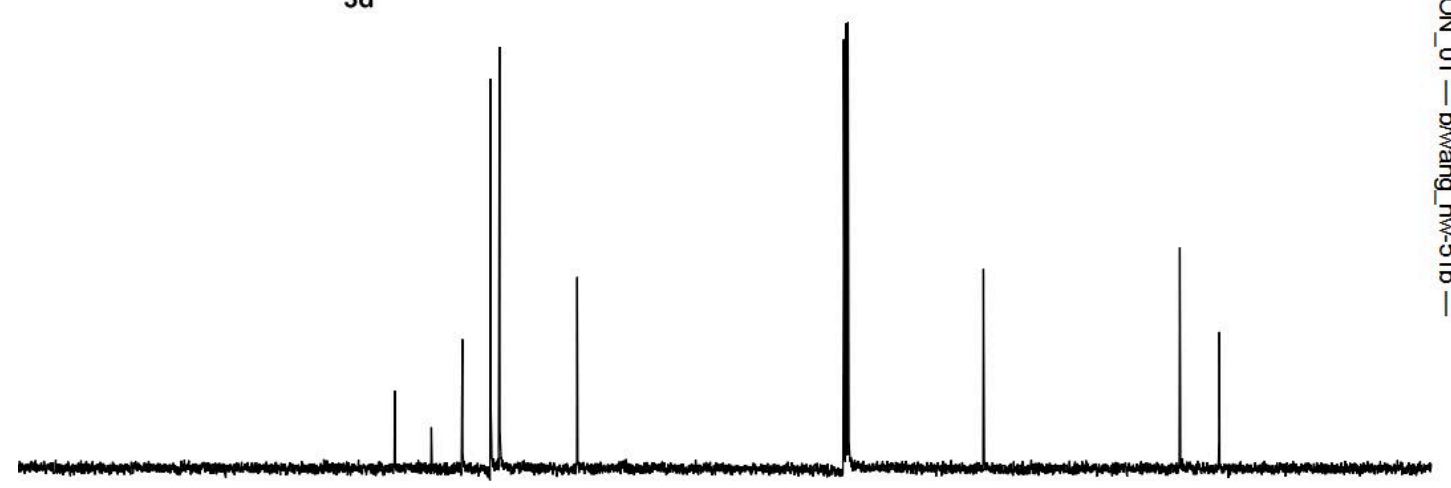

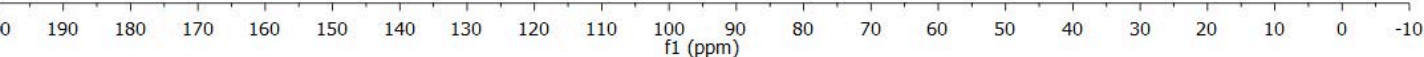


๓

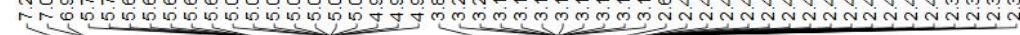
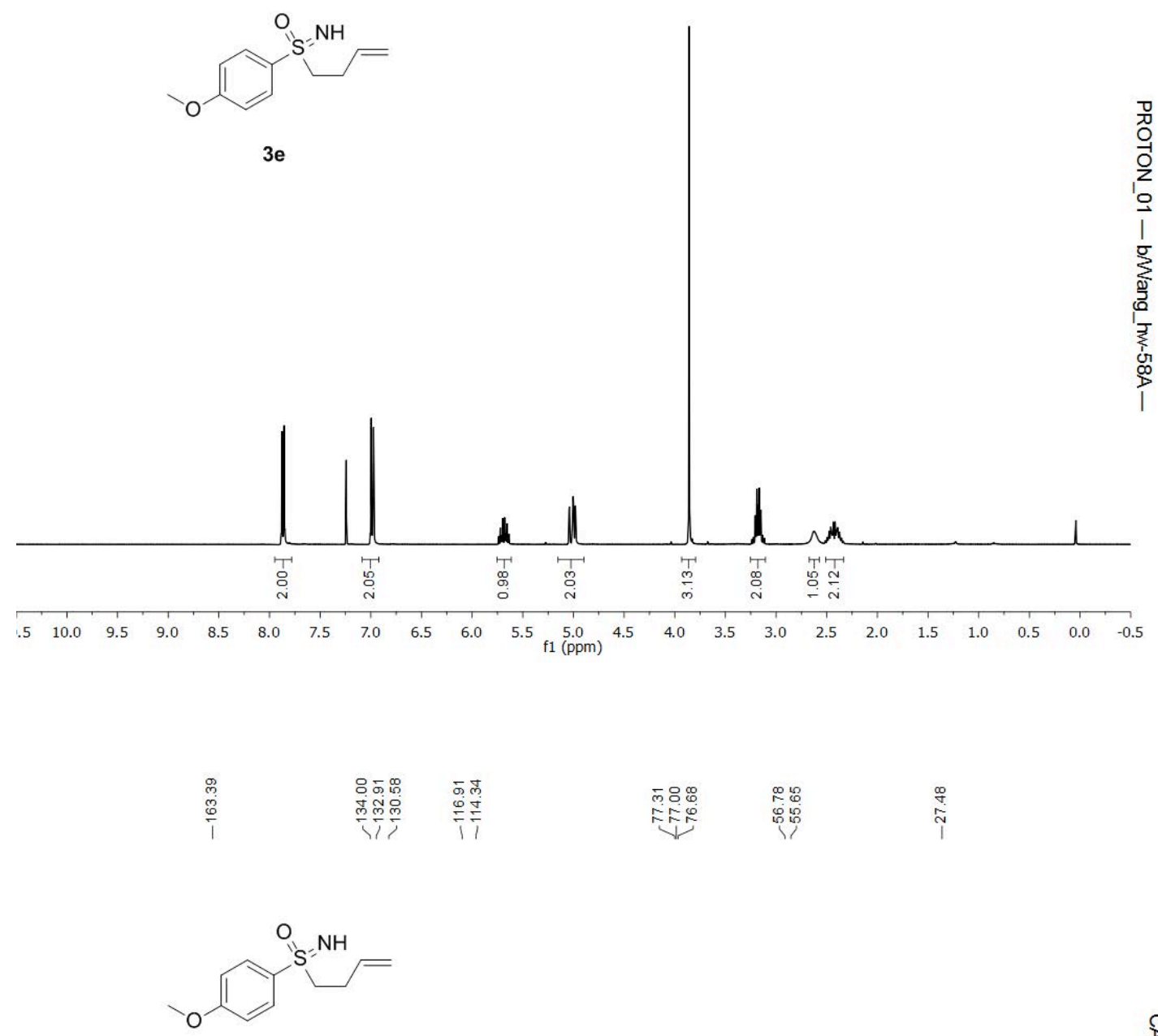

$3 e$ 


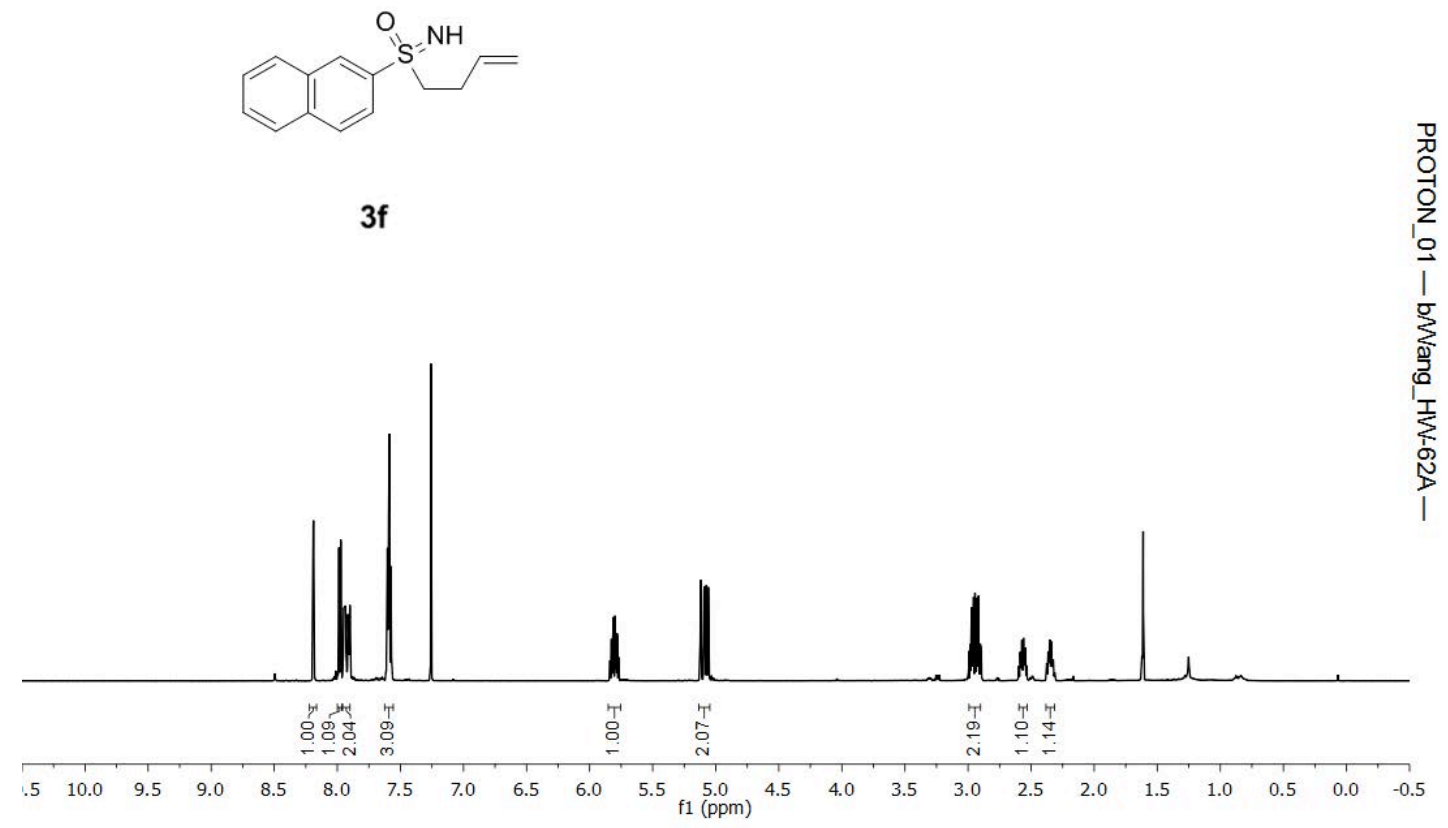

이에

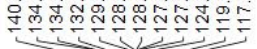

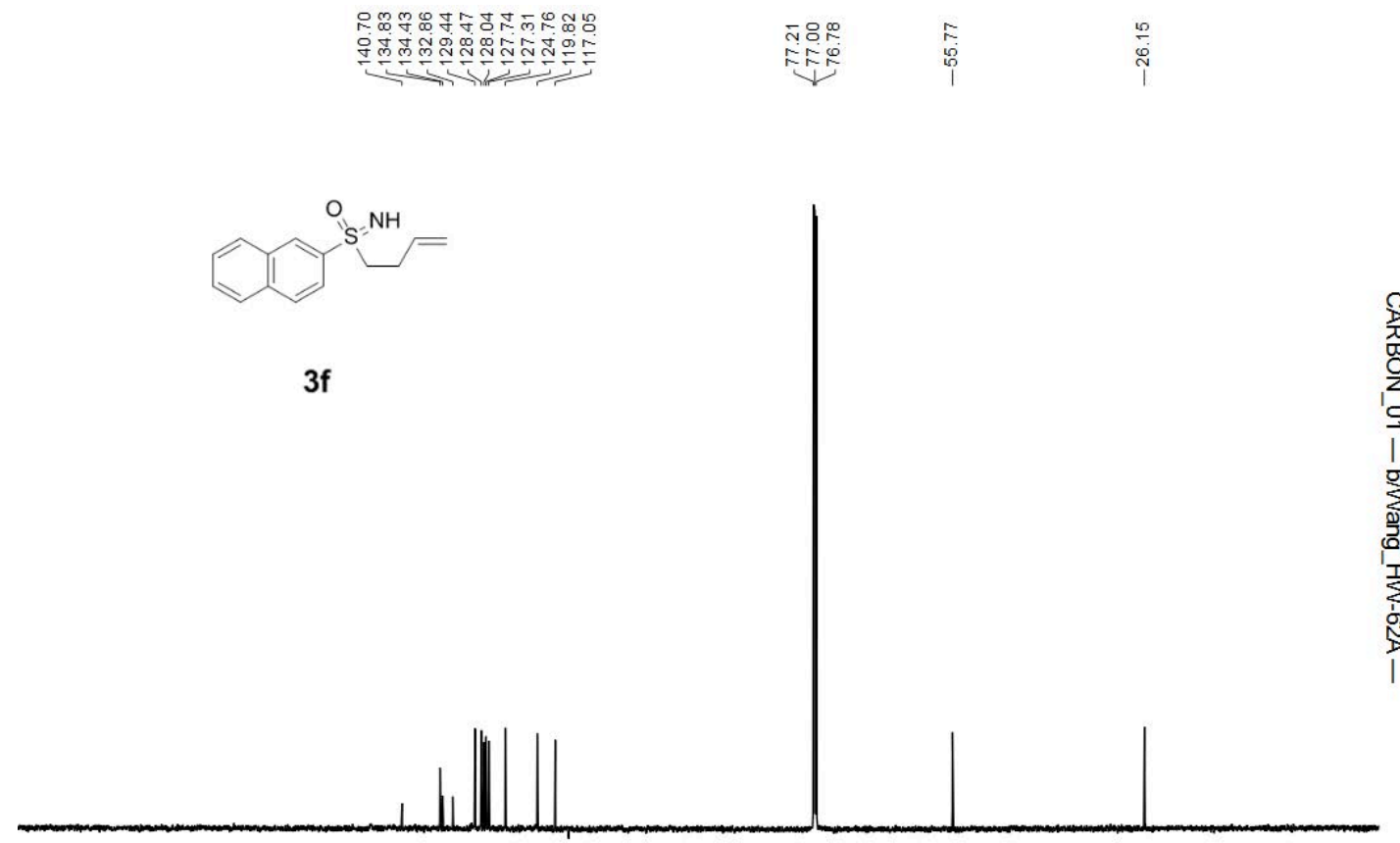

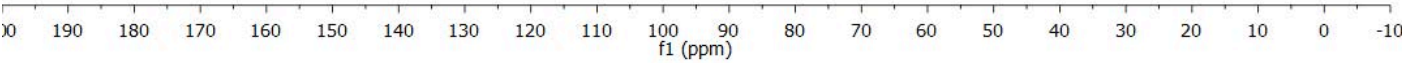


等

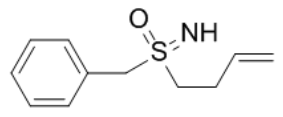

$3 g$
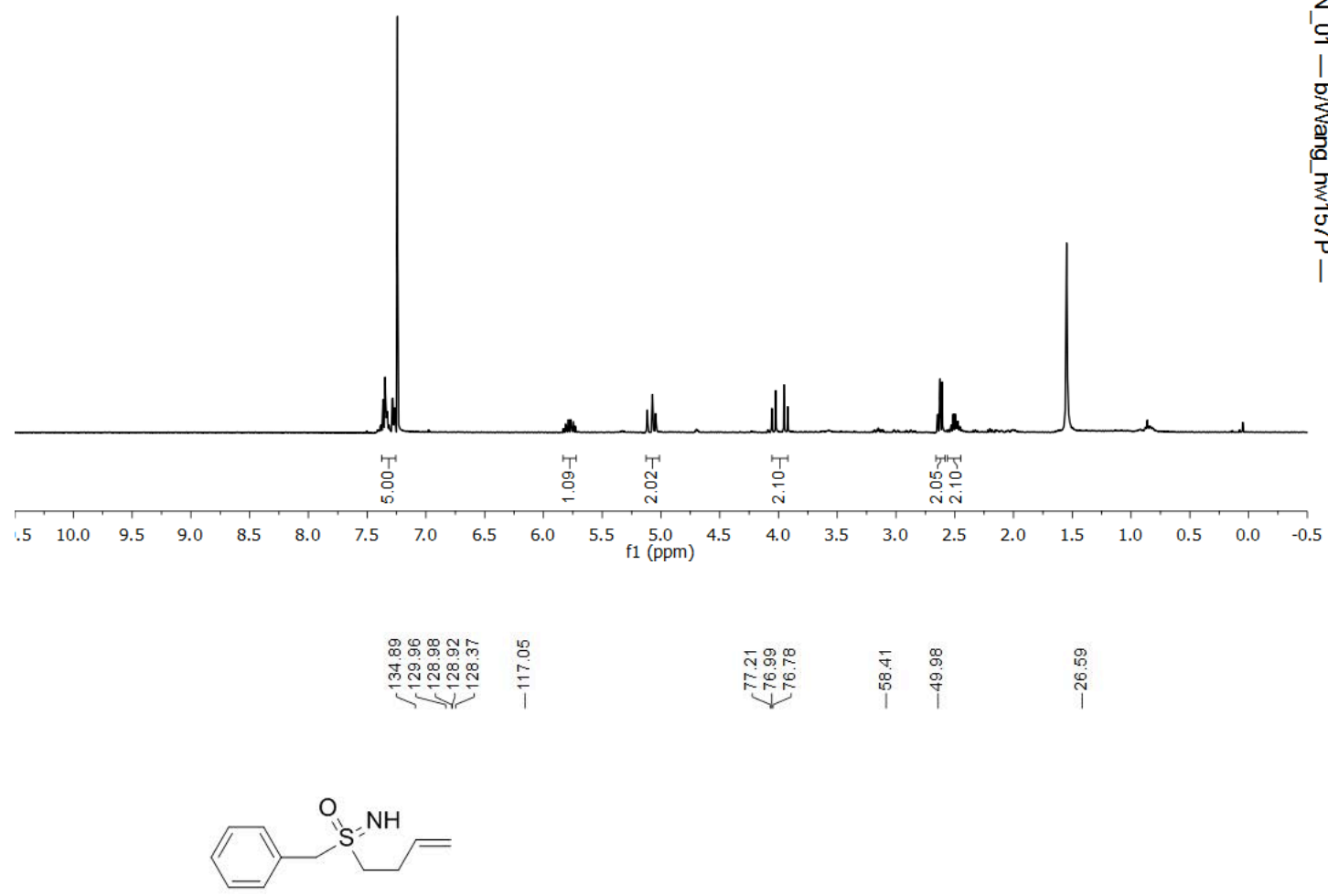

$3 g$

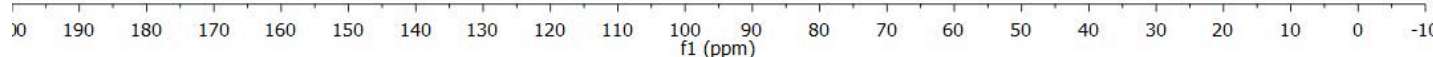




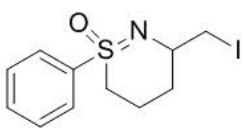

$2 \mathrm{a}$
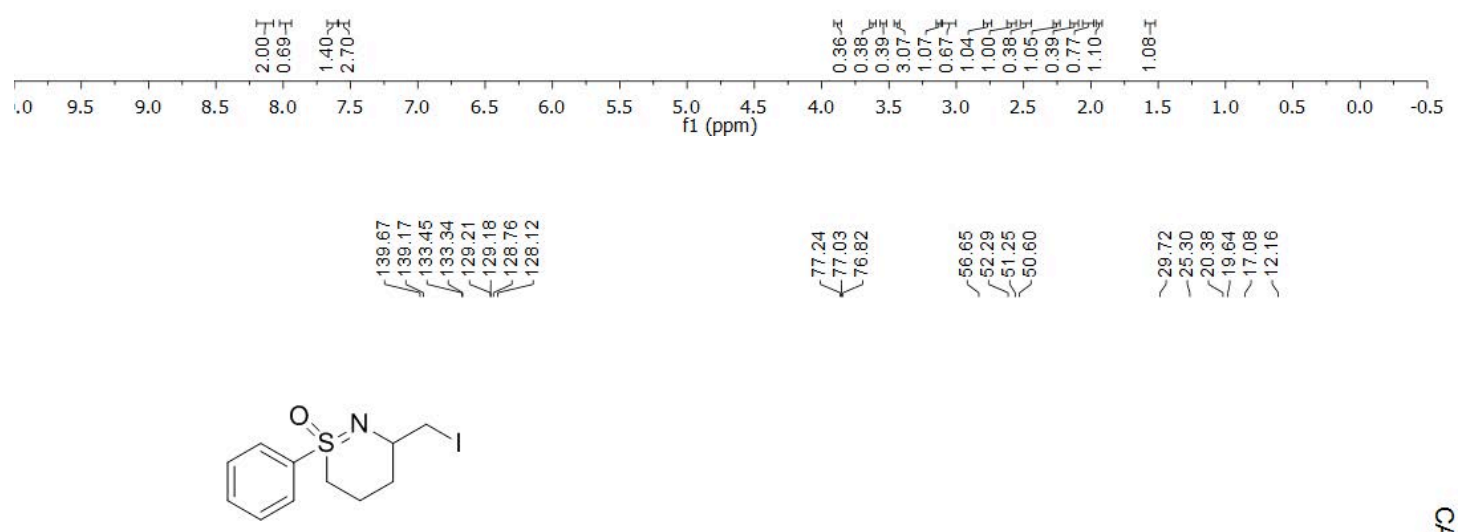

$2 \mathrm{a}$ 


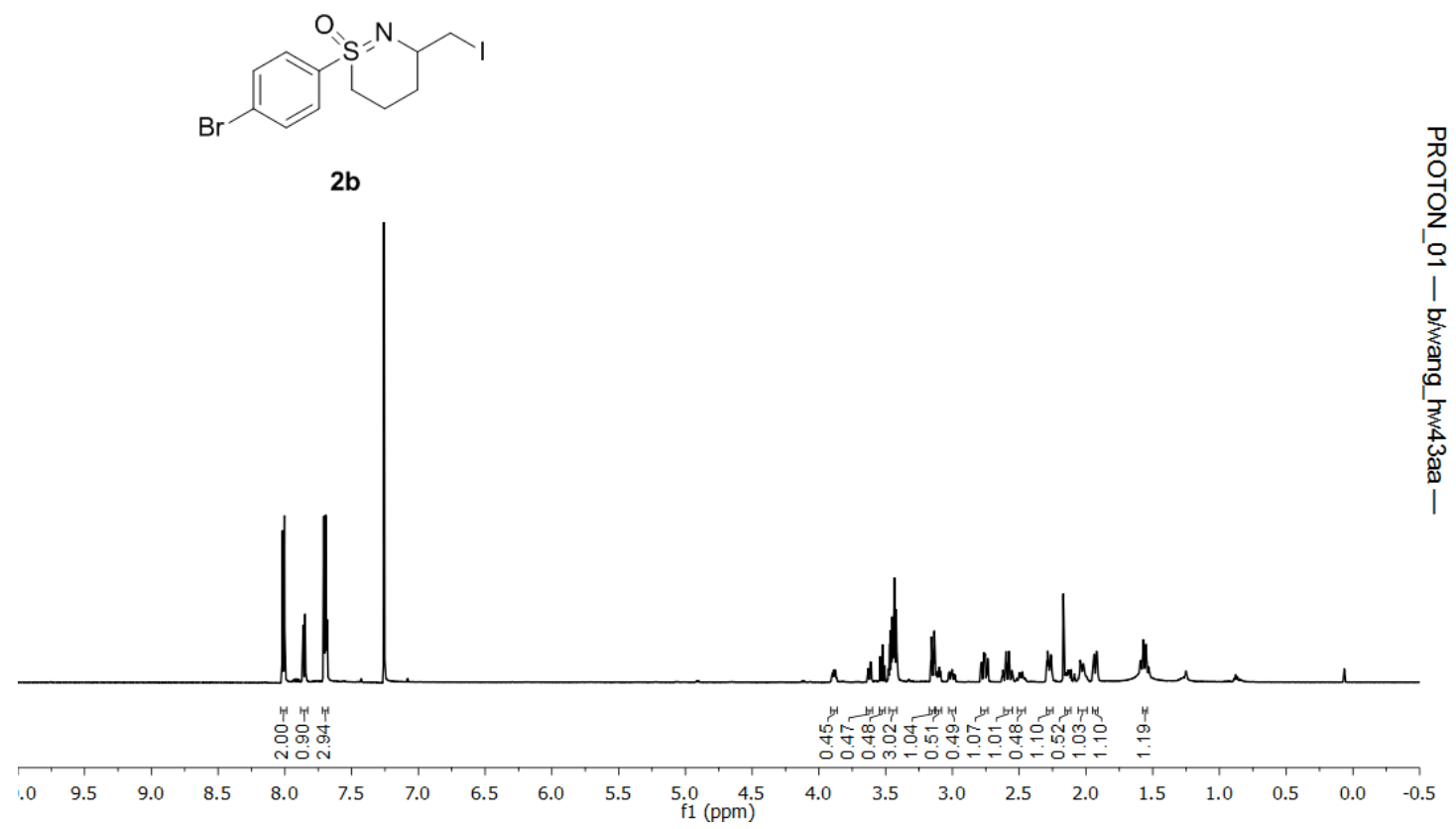

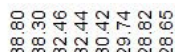

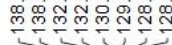

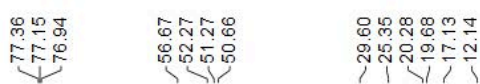

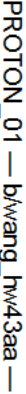<smiles>O=[SH]1(c2ccc(Br)cc2)CCCC(CI)N1</smiles>

$2 b$

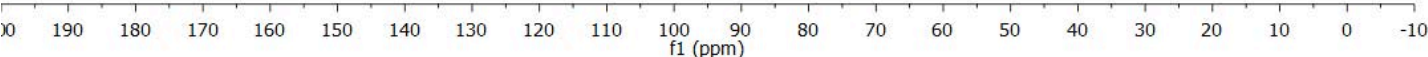



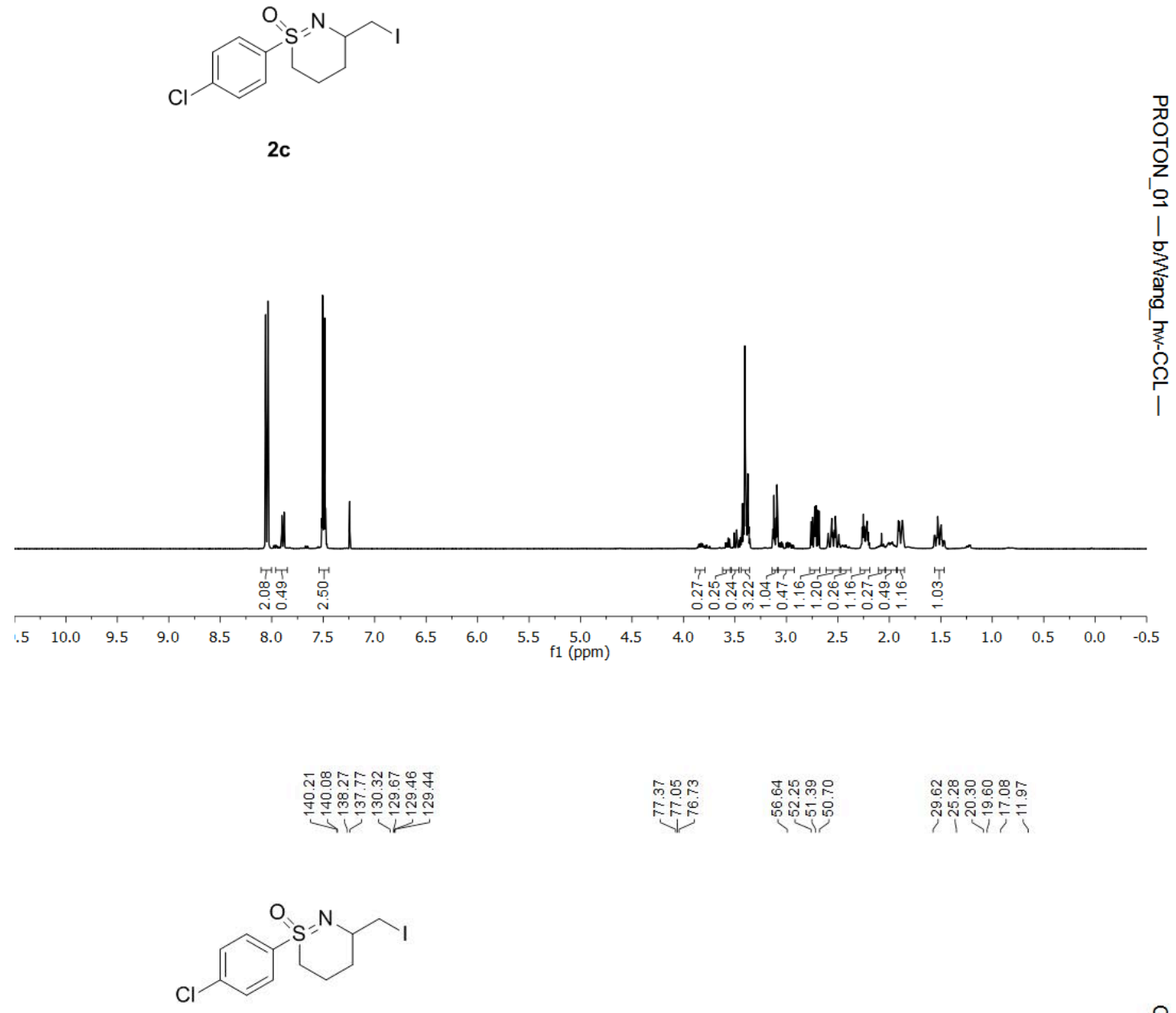

2c 


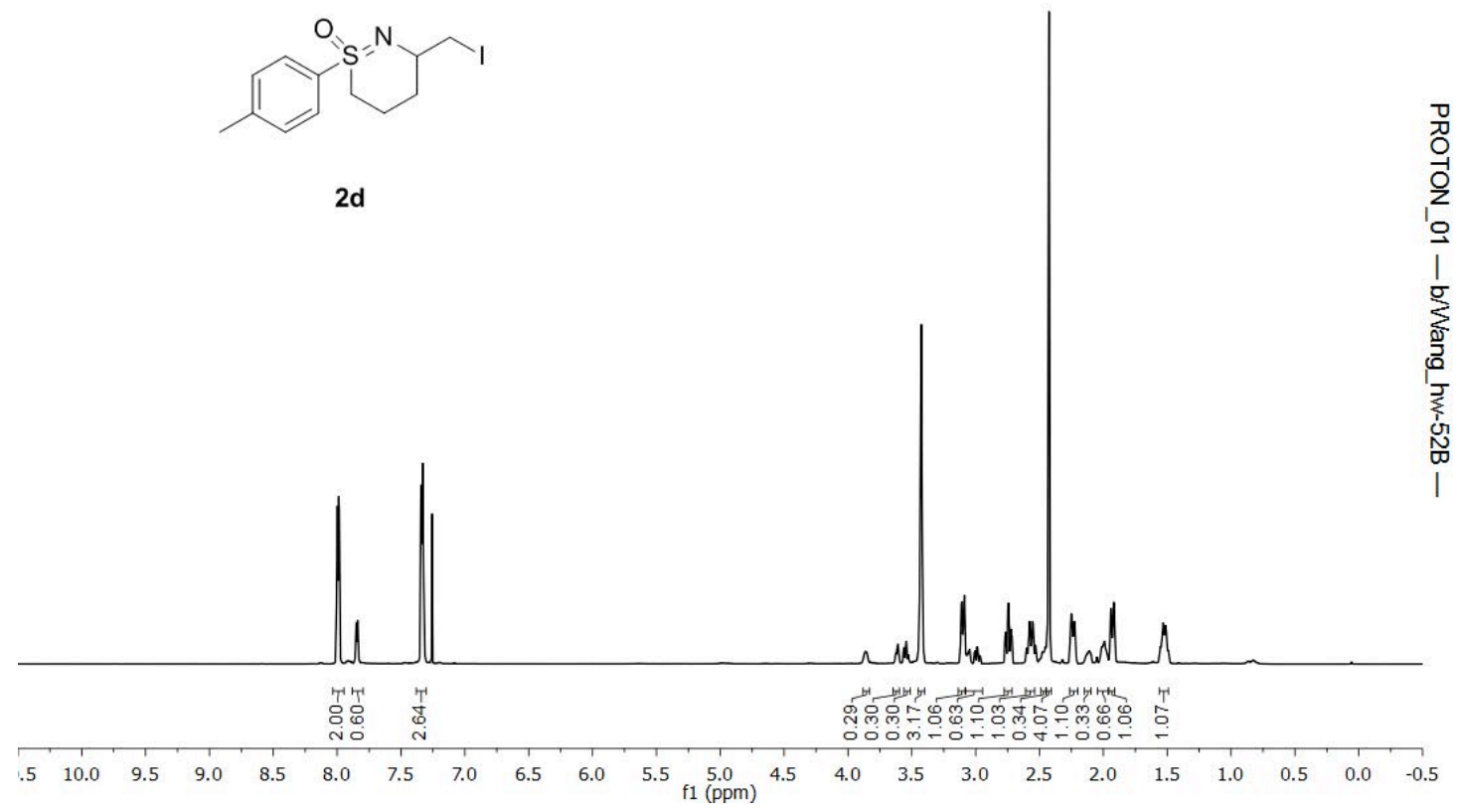

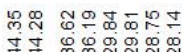

守㠻

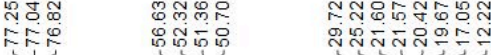

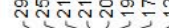

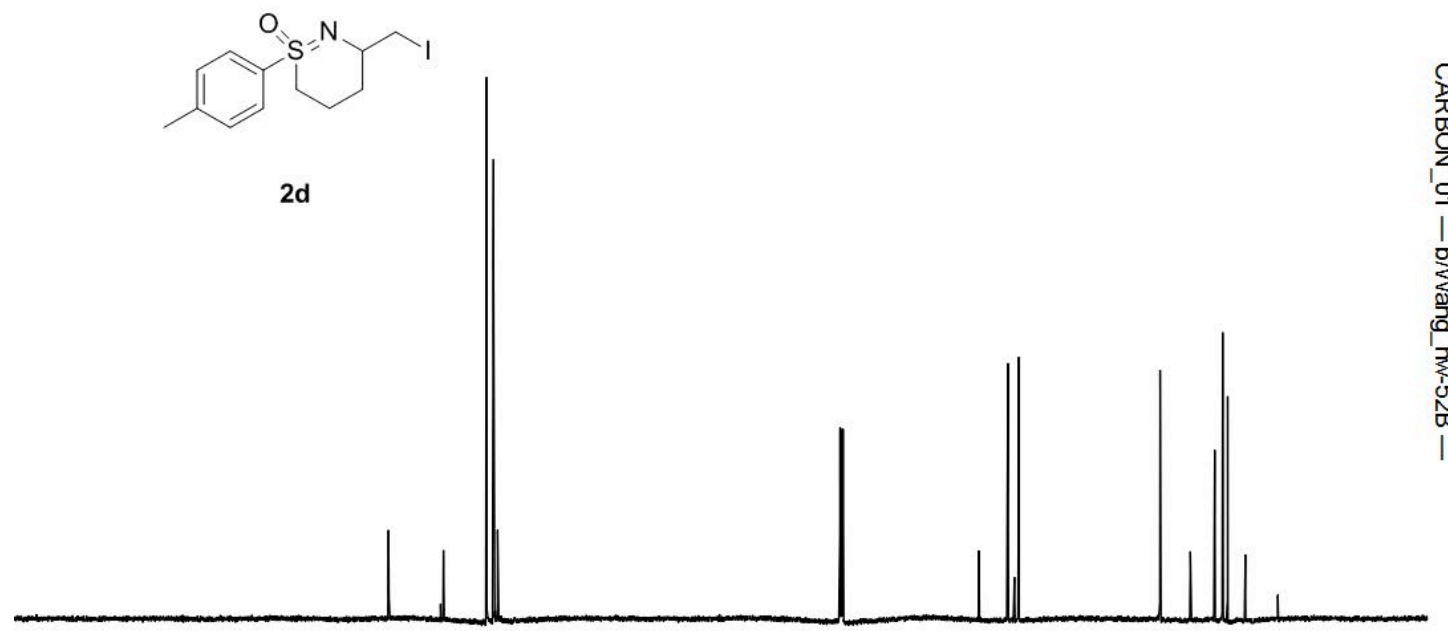

\begin{tabular}{llllllllllllllllllllll}
\hline 0 & 190 & 180 & 170 & 160 & 150 & 140 & 130 & 120 & 110 & $\underset{\mathrm{f} 1}{100}(\mathrm{ppm})$ & 90 & 80 & 70 & 60 & 50 & 40 & 30 & 20 & 10 & 0 & -10
\end{tabular} 


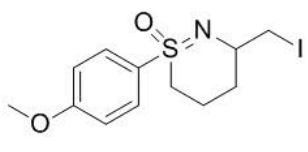

$2 \mathrm{e}$

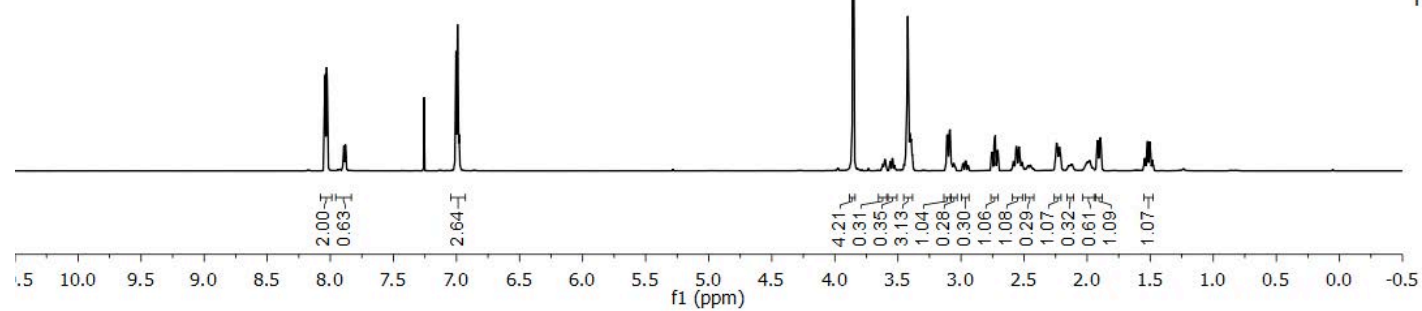

ل年

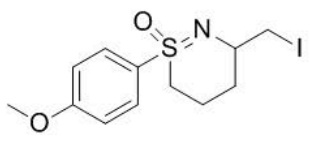

$2 \mathrm{e}$

\begin{tabular}{lllllllllllllllllllllll}
\hline 0 & 190 & 180 & 170 & 160 & 150 & 140 & 130 & 120 & 110 & $\underset{\mathrm{f} 1}{100} \mathrm{(ppm})$ & 90 & 70 & 60 & 50 & 40 & 30 & 20 & 10 & 0 & -10
\end{tabular} 


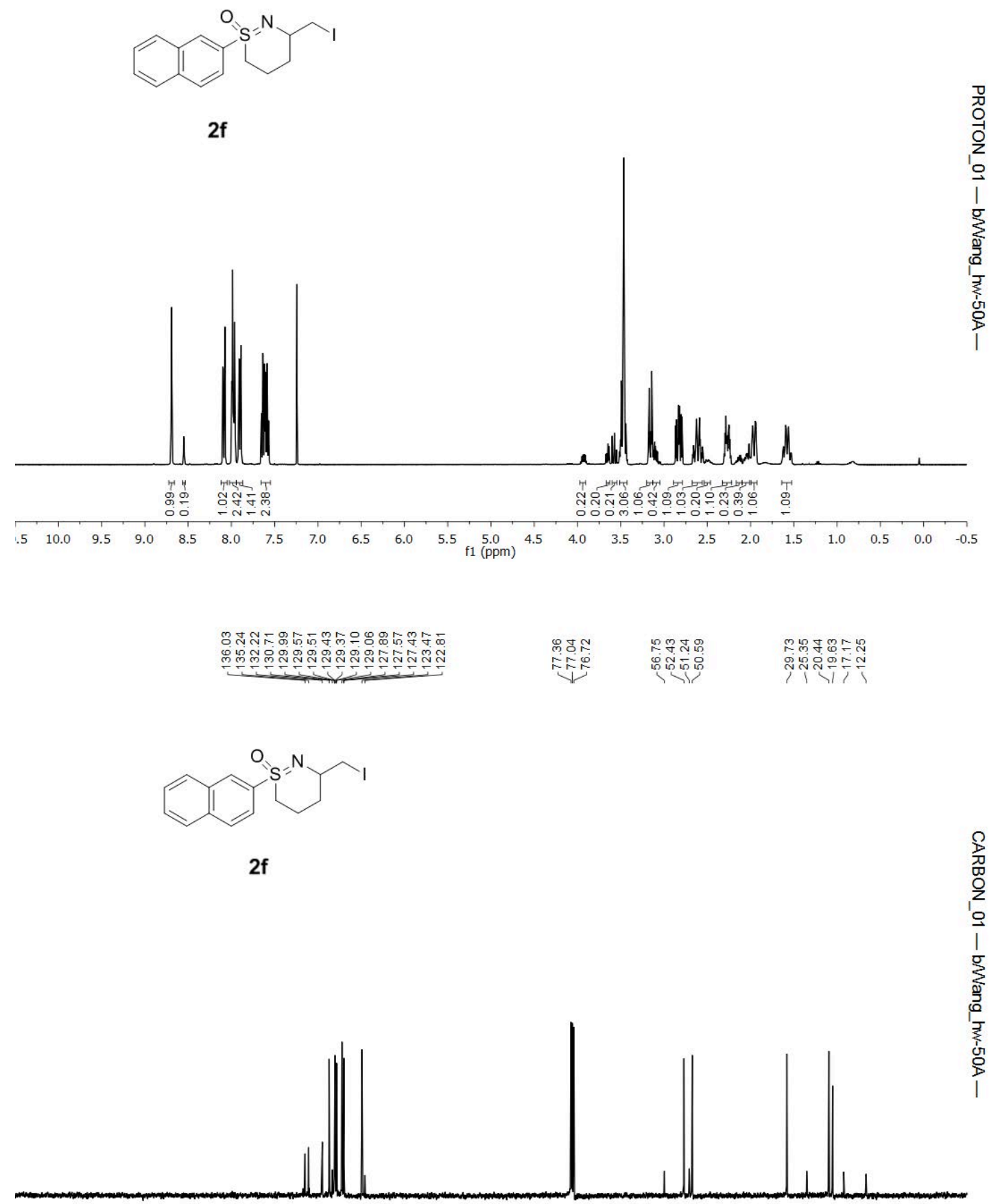

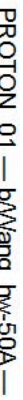




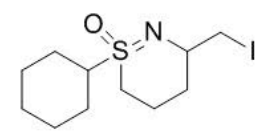

$2 \mathrm{~g}$

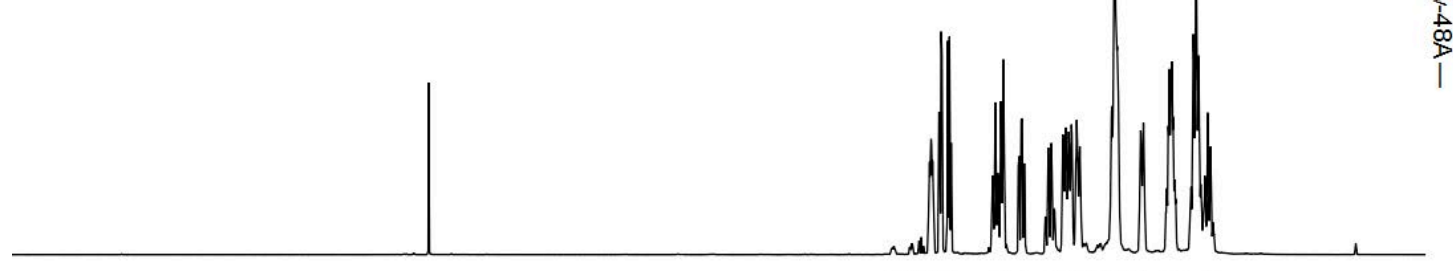

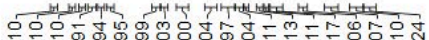

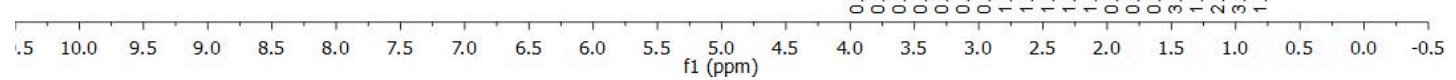

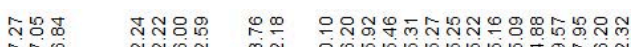

证

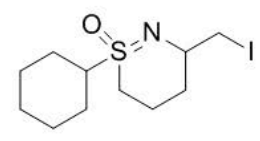

$2 \mathrm{~g}$

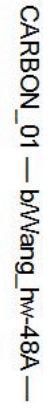

$\begin{array}{llllllllllllllllllllllllll}10 & 190 & 180 & 170 & 160 & 150 & 140 & 130 & 120 & 110 & 100 & 90 & 80 & 70 & 60 & 50 & 40 & 30 & 20 & 10 & 0 & -10\end{array}$ 


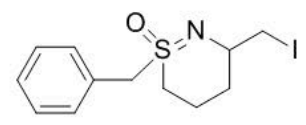

$2 \mathrm{~h}$

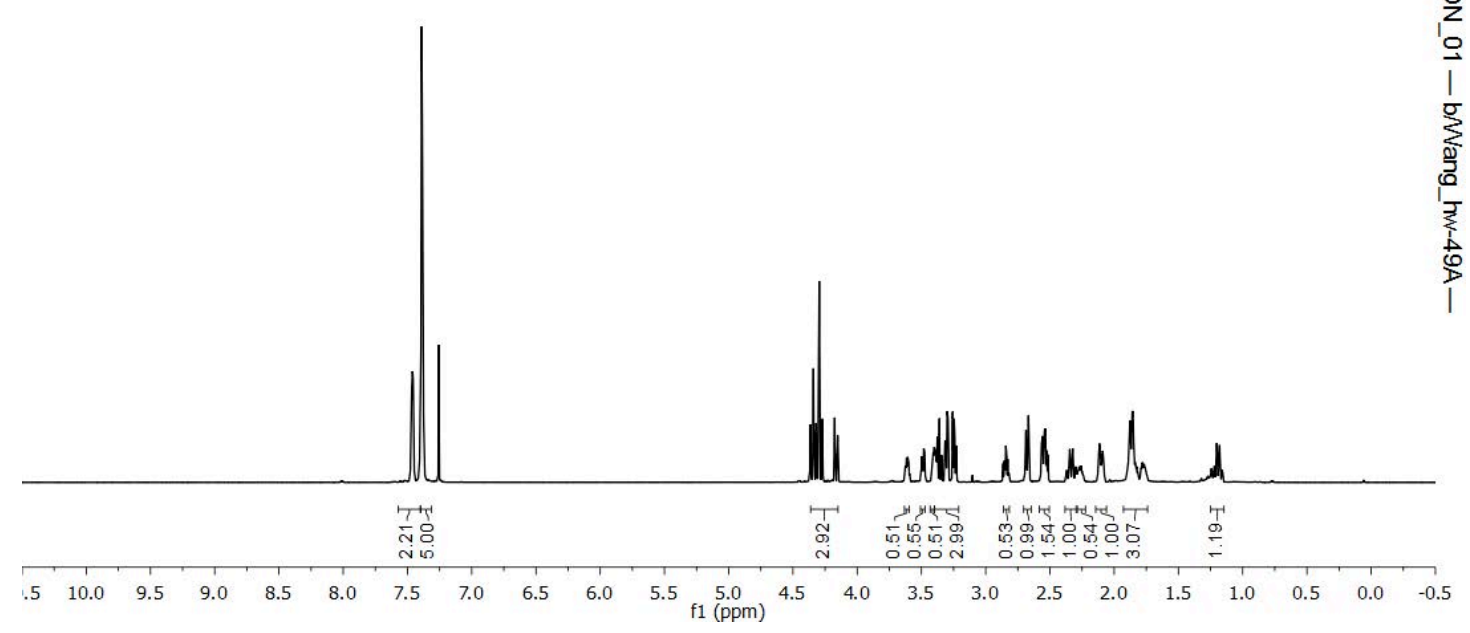

8 \&:

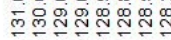

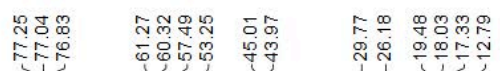

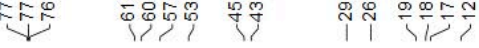

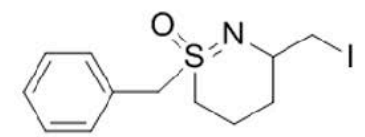

$2 \mathrm{~h}$

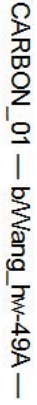

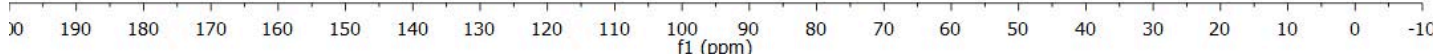




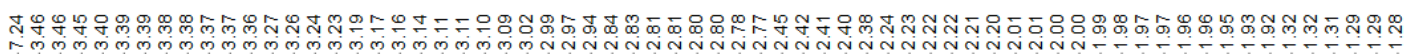

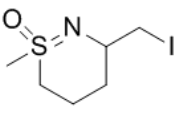

2i

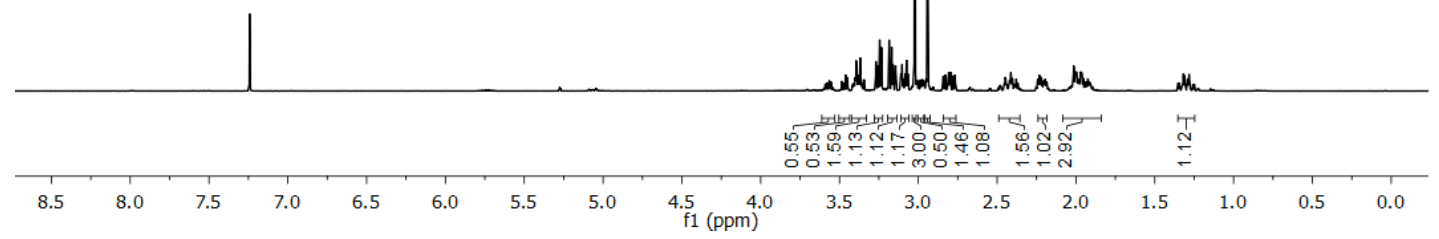

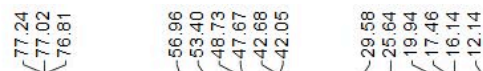

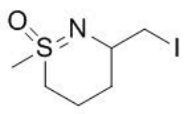

$2 \mathbf{i}$

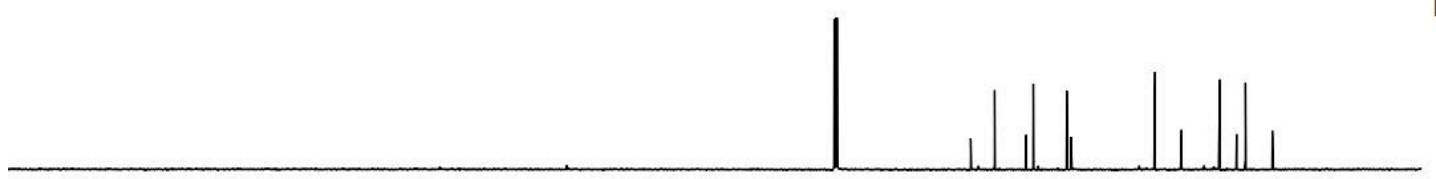

$\begin{array}{llllllllllllllllllllll}10 & 190 & 180 & 170 & 160 & 150 & 140 & 130 & 120 & 110 & 100 & 90 & 80 & 70 & 60 & 50 & 40 & 30 & 20 & 10 & 0 & -10\end{array}$ 


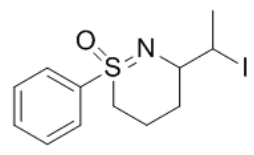

2j
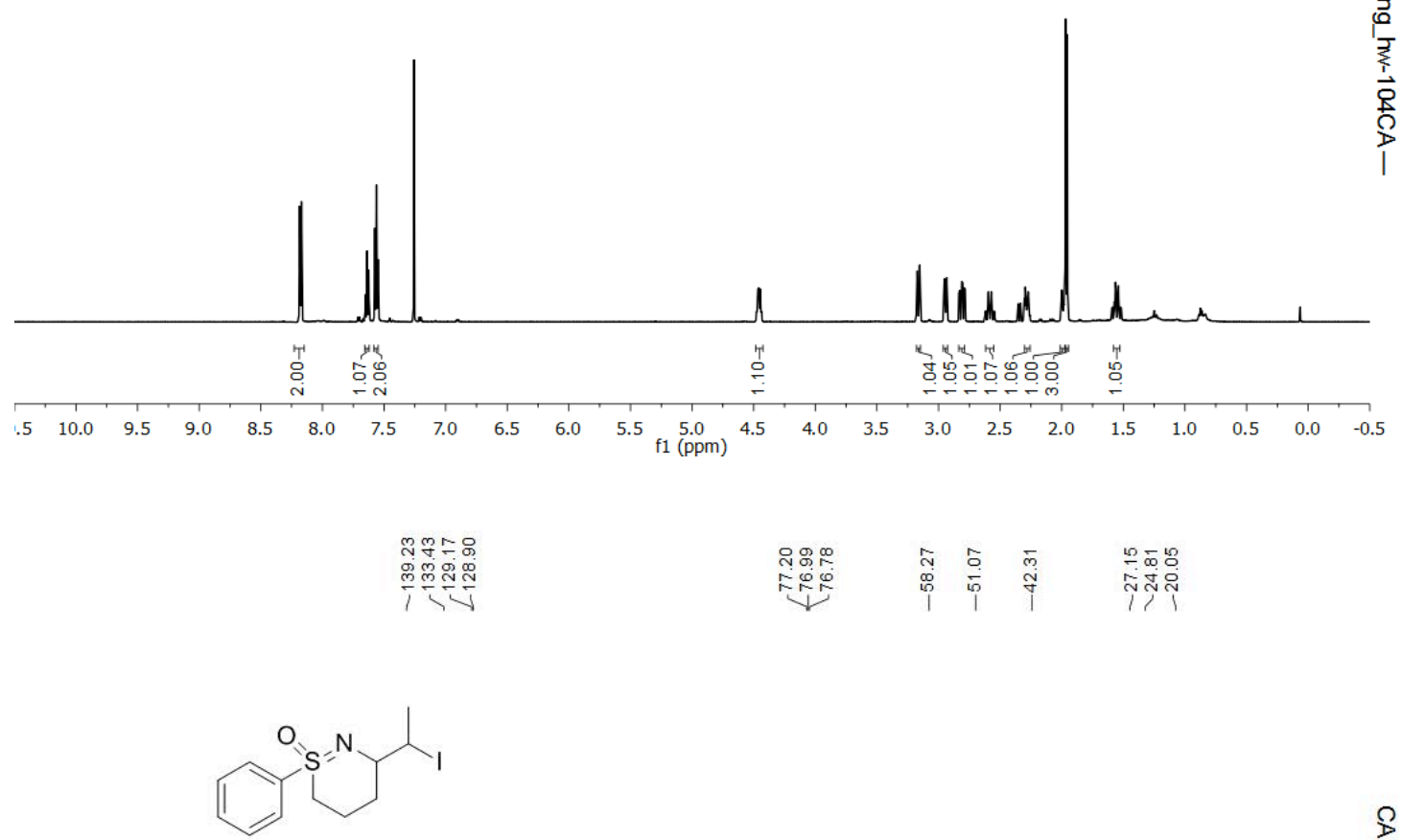

$2 \mathrm{j}$

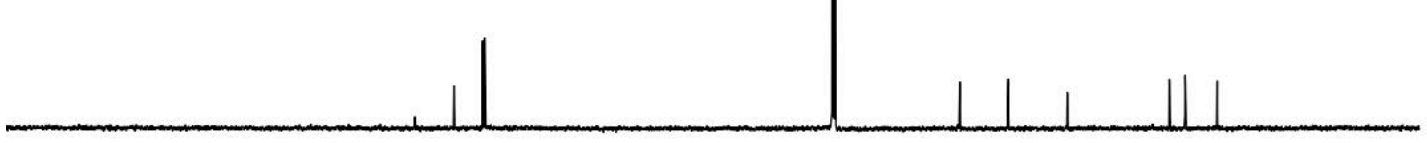

$\begin{array}{llllllllllllllllllllll}10 & 190 & 180 & 170 & 160 & 150 & 140 & 130 & 120 & 110 & \begin{array}{l}100 \\ \mathrm{f} 1(\mathrm{ppm})\end{array} & 80 & 70 & 60 & 50 & 40 & 30 & 20 & 10 & 0 & -10\end{array}$ 

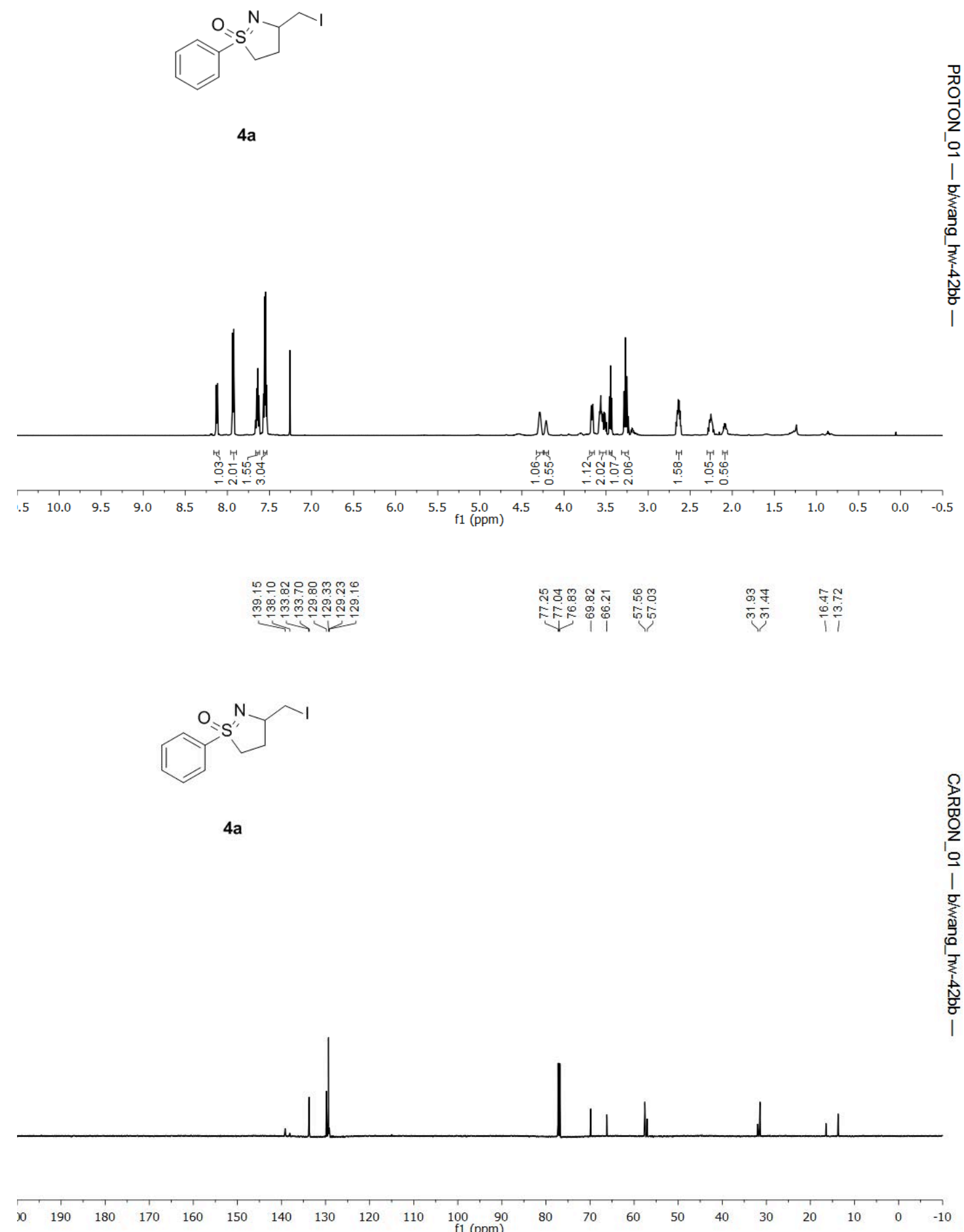


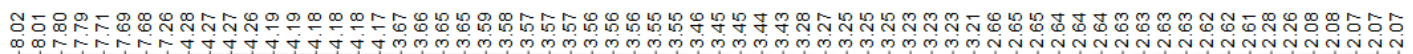

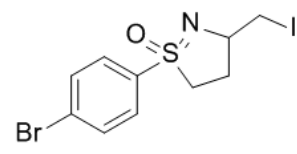

4b

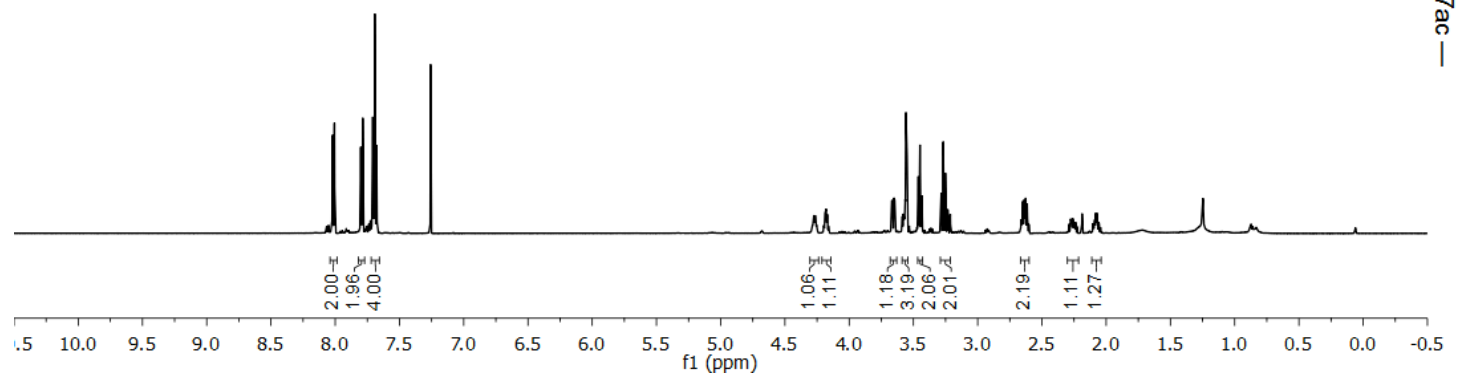

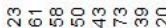

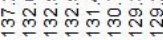

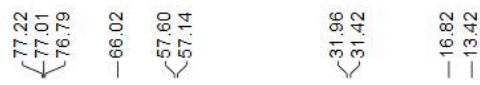

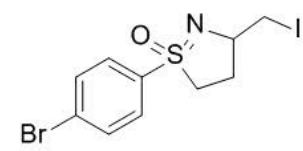

$4 b$

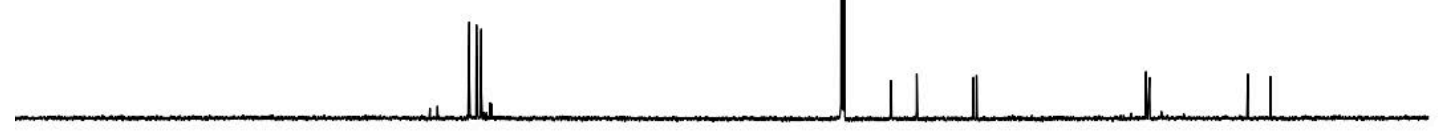

$\begin{array}{llllllllllllllllllllll}10 & 190 & 180 & 170 & 160 & 150 & 140 & 130 & 120 & 110 & 100 & 90 & 80 & 70 & 60 & 50 & 40 & 30 & 20 & 10 & 0 & -10\end{array}$ 


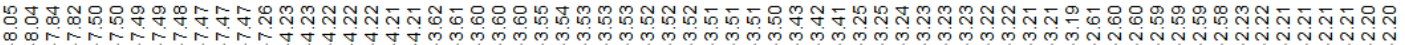

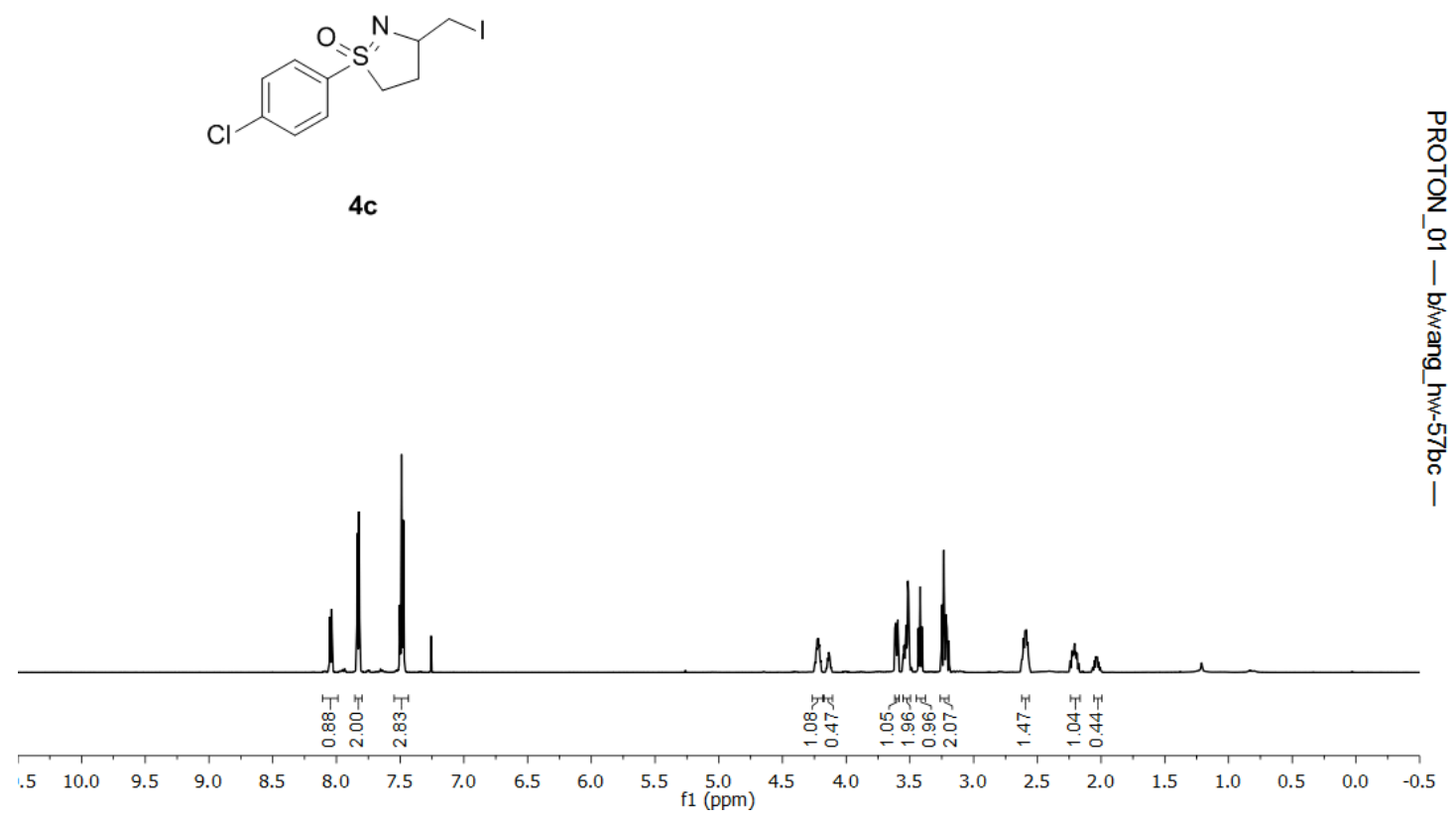

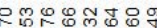

뭄쓴 은

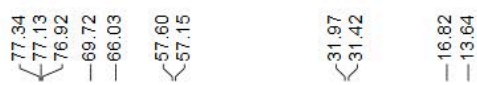

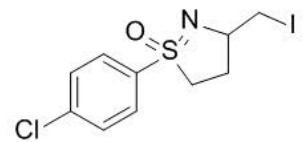

4c

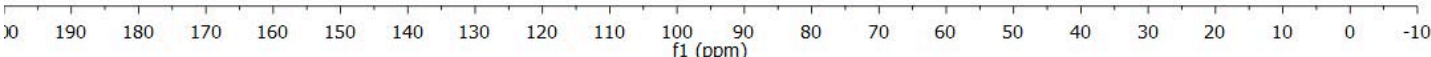




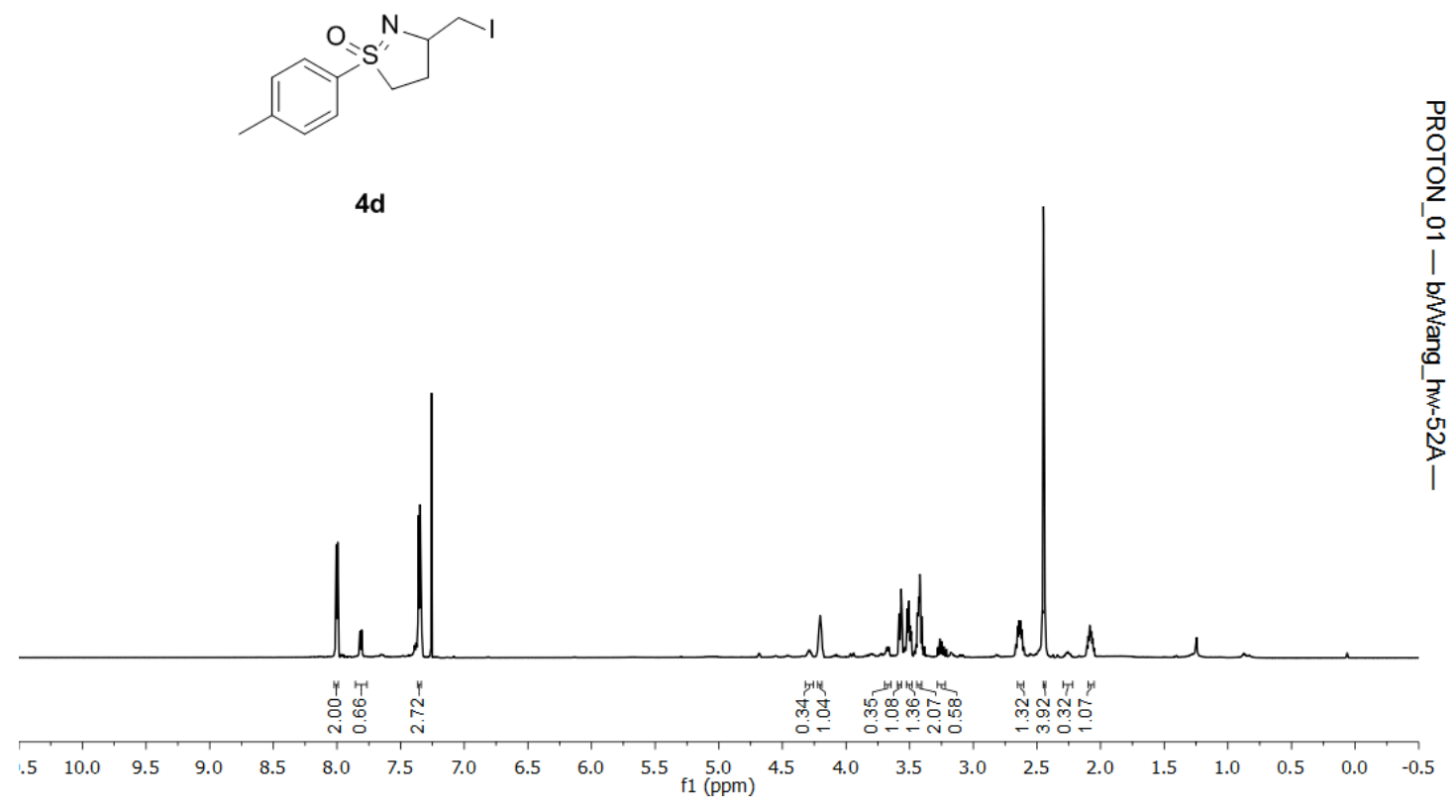

๓

莳

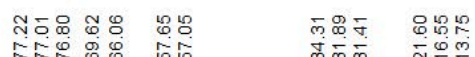

婇

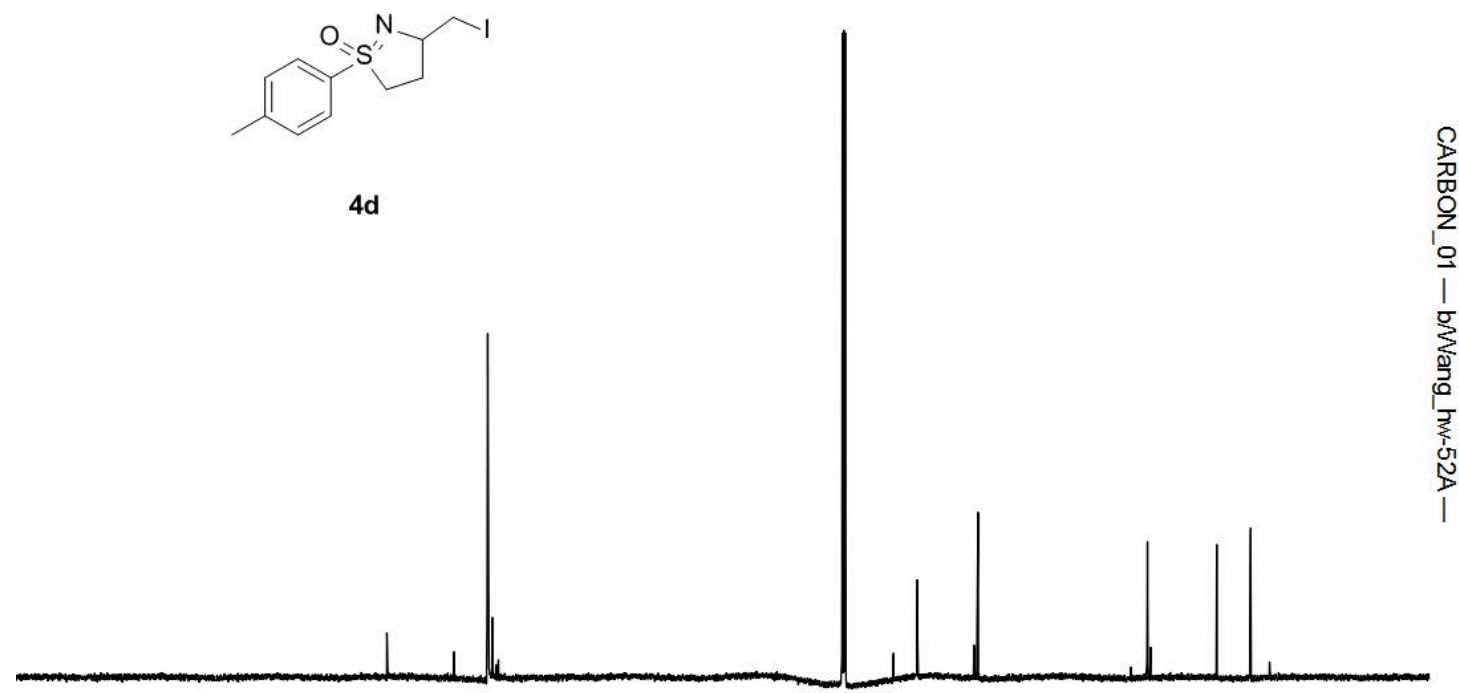

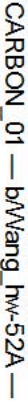

$\begin{array}{lllllllllllllllllllllllll}10 & 190 & 180 & 170 & 160 & 150 & 140 & 130 & 120 & 110 & 100 & 90 & 80 & 70 & 60 & 50 & 40 & 30 & 20 & 10 & 0 & -10\end{array}$ 

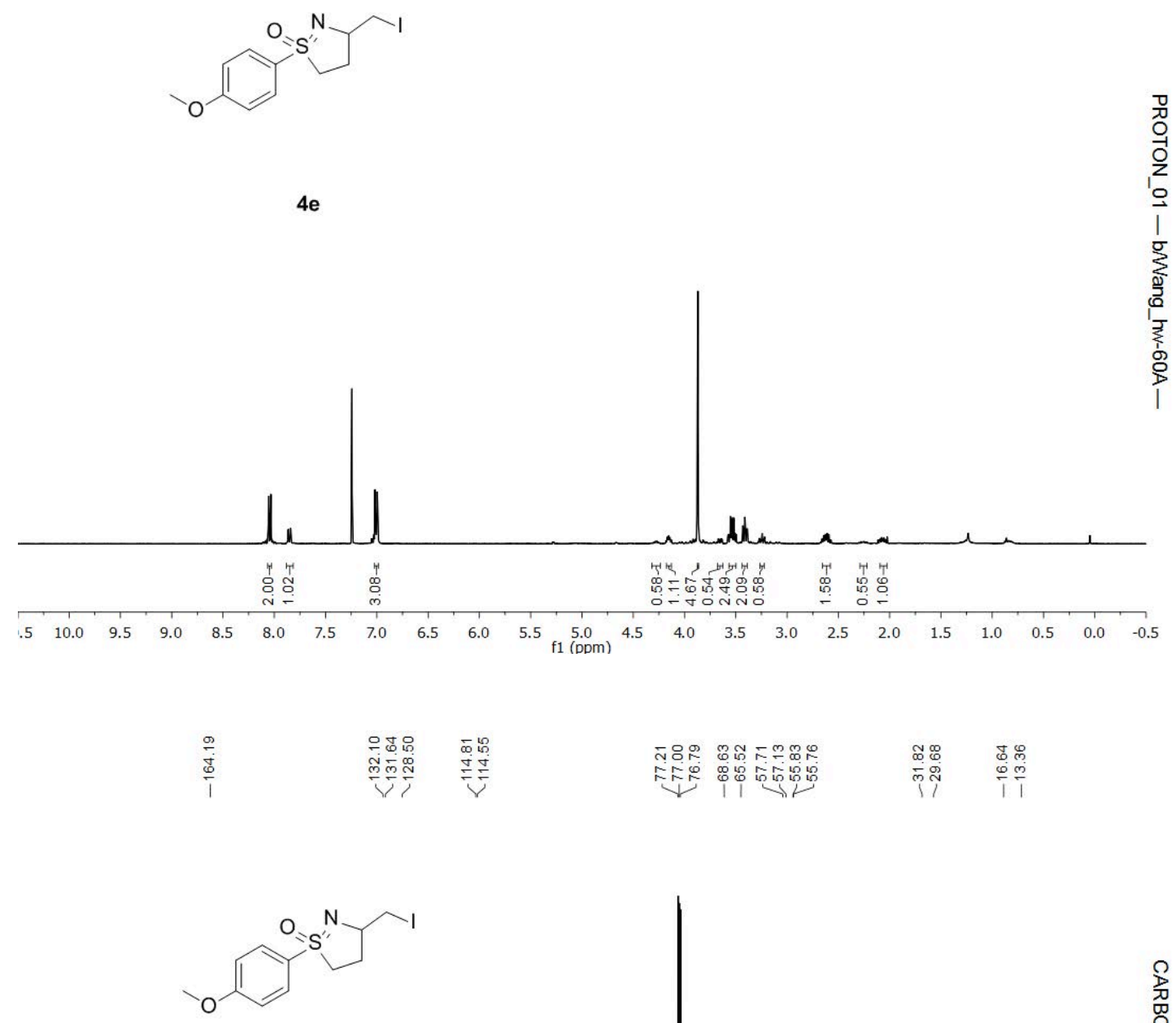

$4 \mathrm{e}$ 


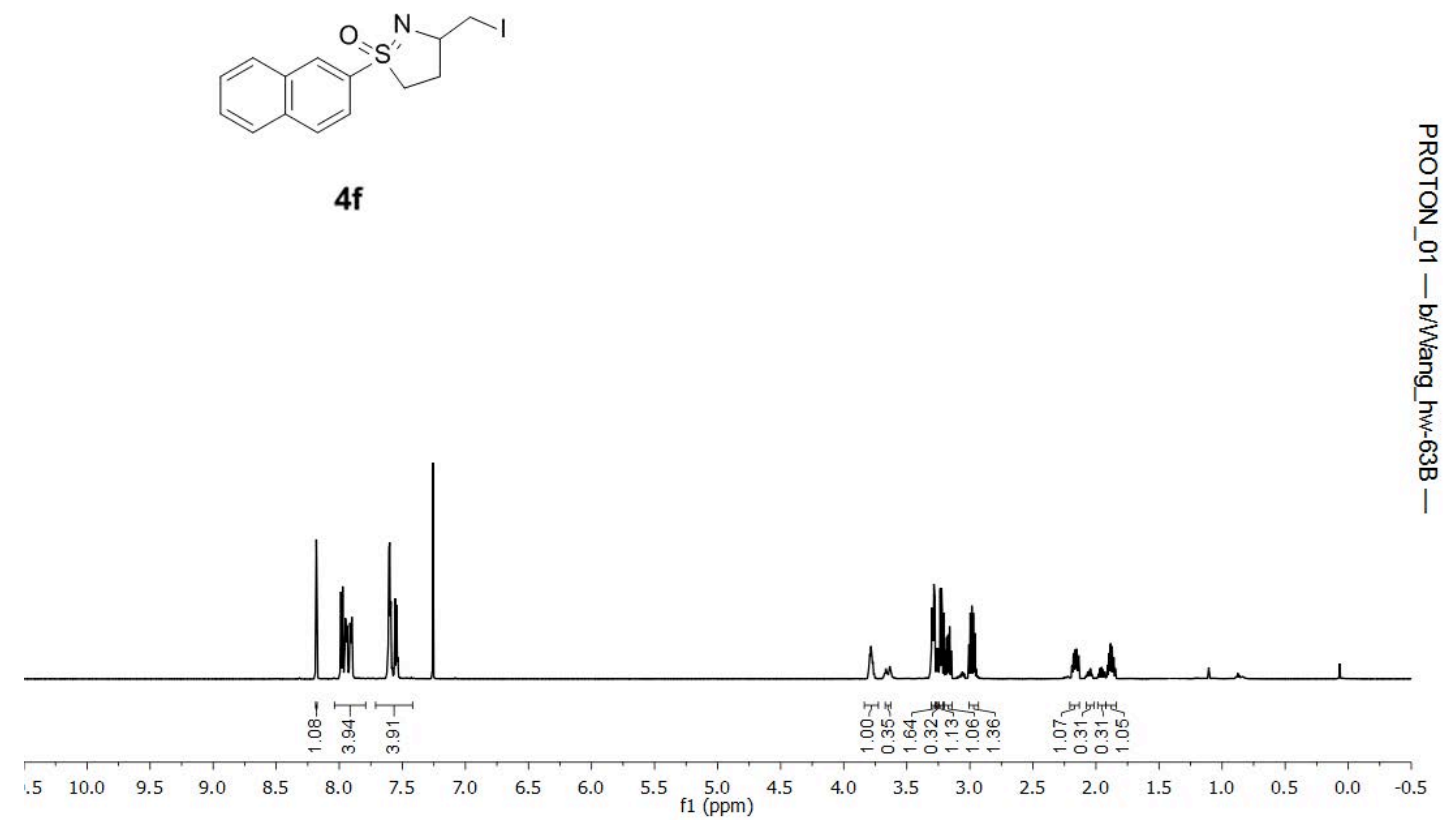

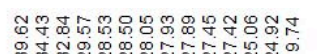

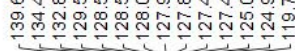

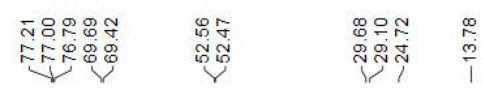

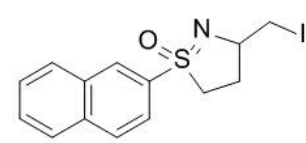

$4 f$ 

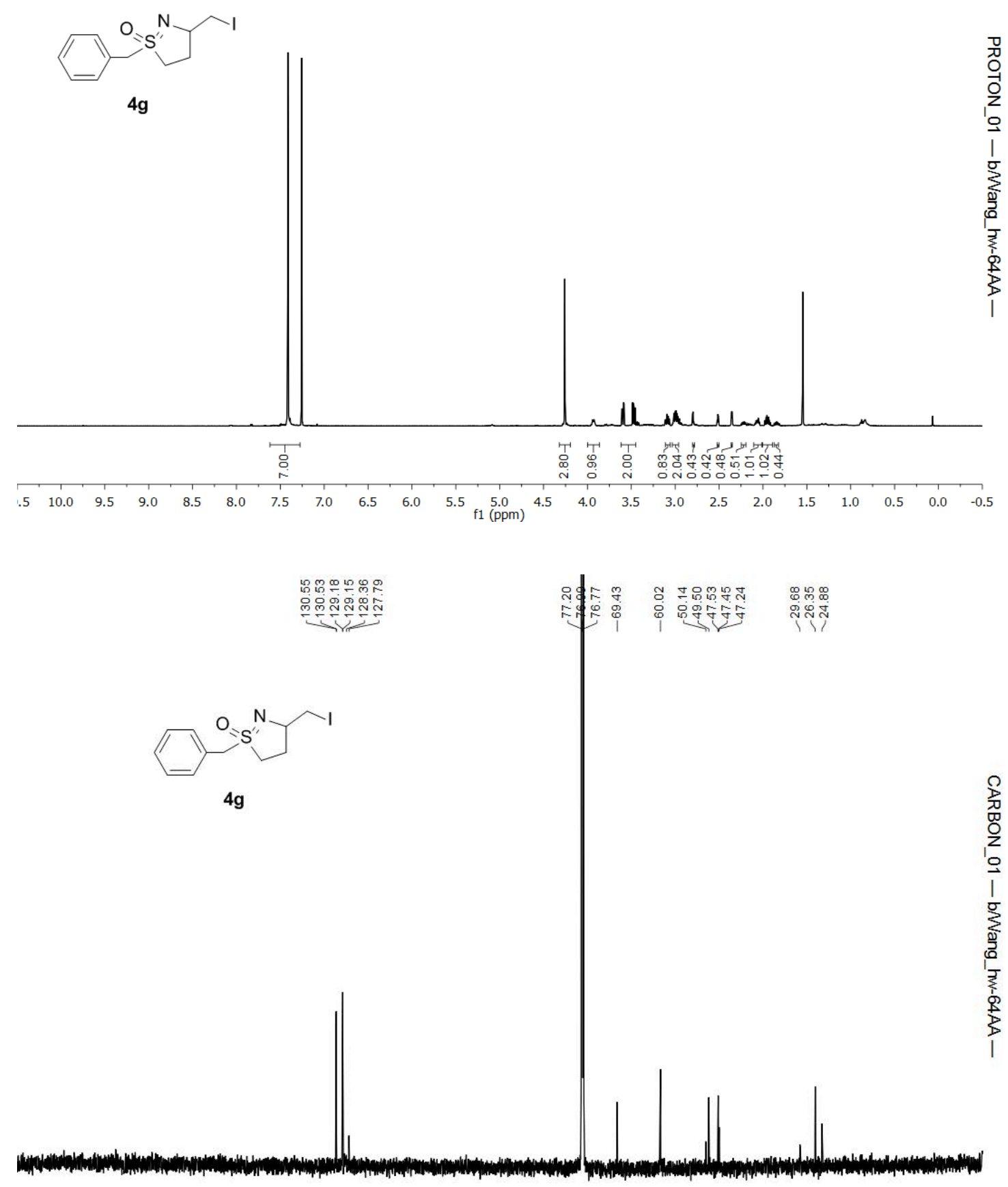

$\begin{array}{lllllllllllllllllllllllllllllllll}10 & 190 & 180 & 170 & 160 & 150 & 140 & 130 & 120 & 110 & \begin{array}{l}100 \\ \mathrm{f} 1\end{array}(\mathrm{ppm}) & 90 & 80 & 70 & 60 & 50 & 40 & 30 & 20 & 10 & 0 & -10\end{array}$ 
<smiles>O=[SH]1(c2ccccc2)CCCC(Cc2ccccc2)N1</smiles>

5

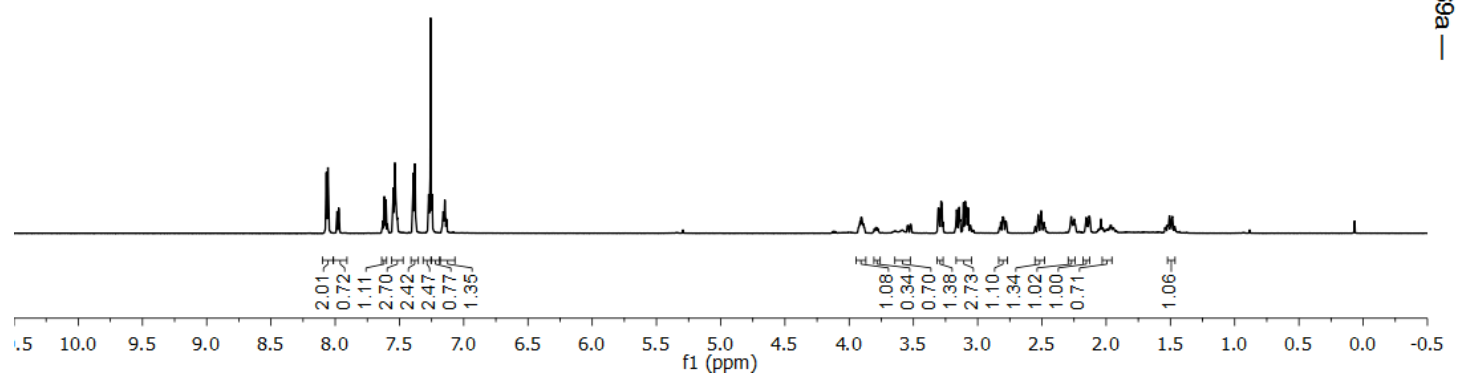

急

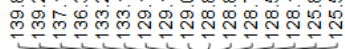

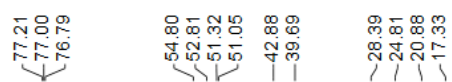

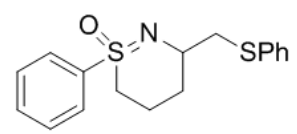

5
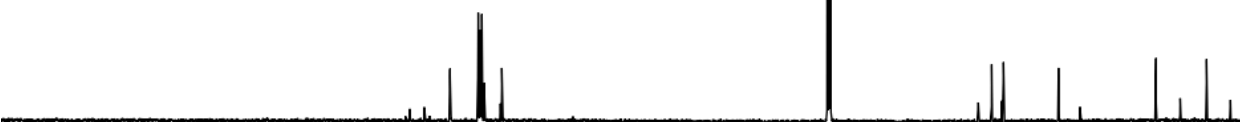

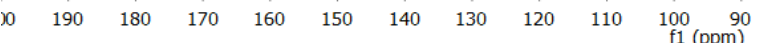

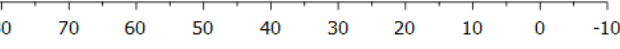




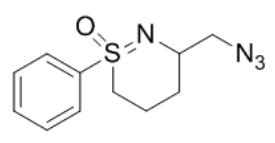

6
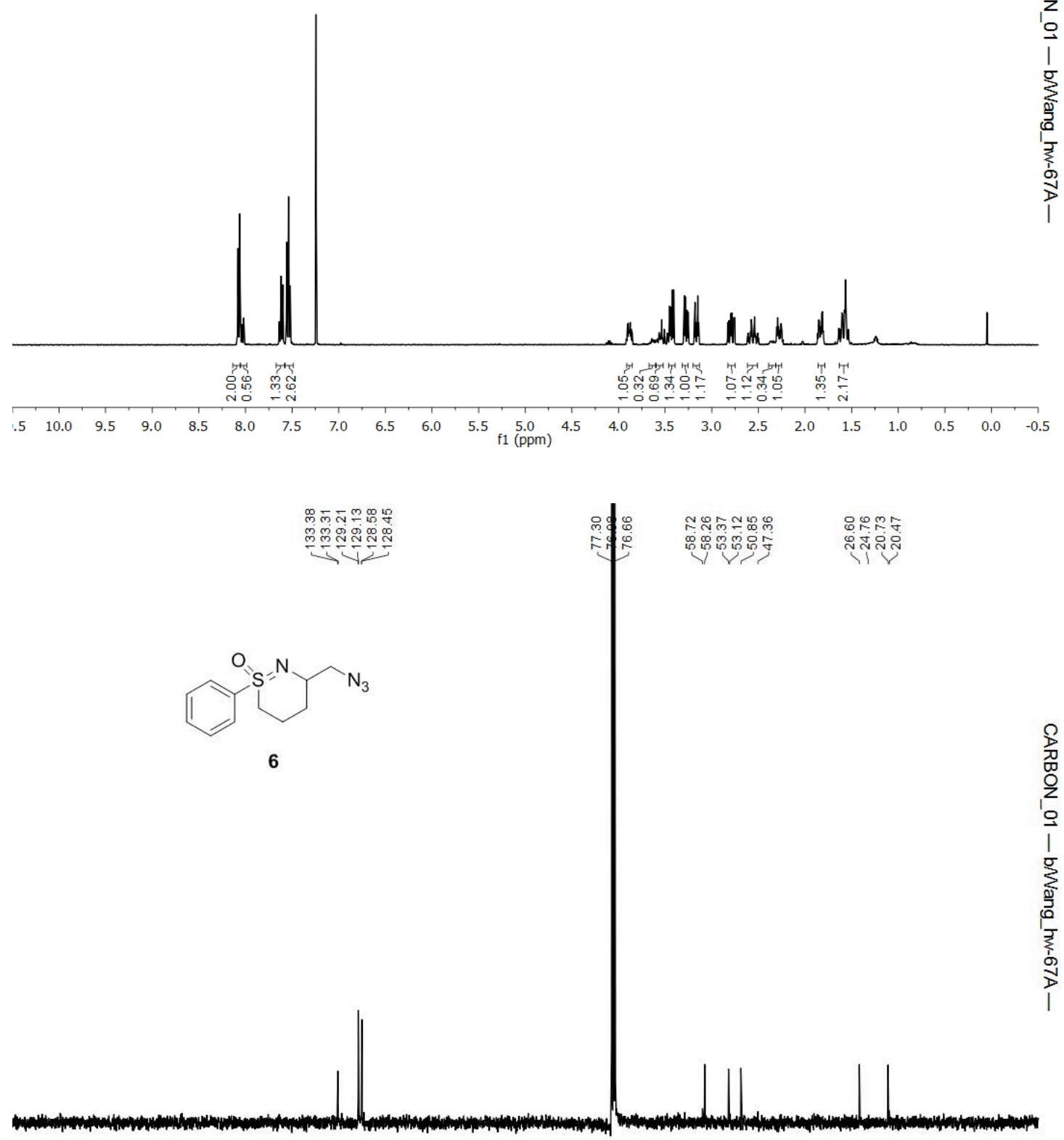

$\begin{array}{llllllllllllllllllllllll}10 & 190 & 180 & 170 & 160 & 150 & 140 & 130 & 120 & 110 & \underset{f 1}{100}(\mathrm{ppm}) & 80 & 70 & 60 & 50 & 40 & 30 & 20 & 10 & 0 & -10\end{array}$ 


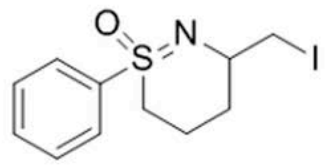

Major diastereomer of $\mathbf{2 a}$

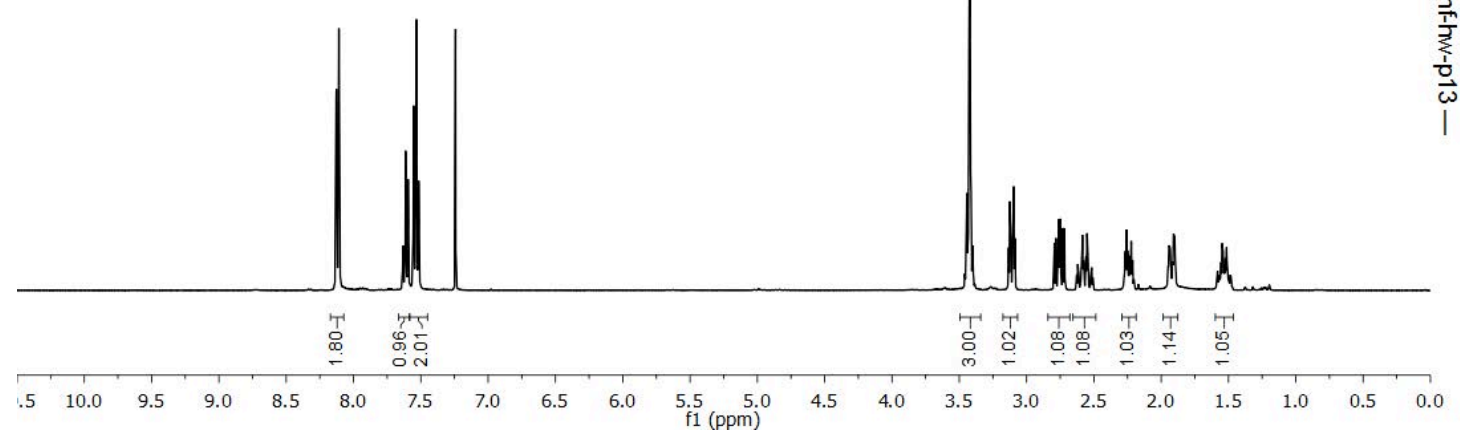

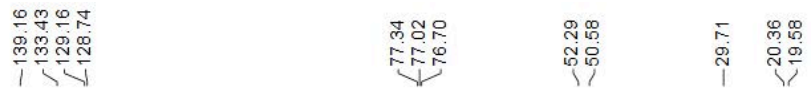<smiles>O=S1(c2ccccc2)=NC(CI)CCC1</smiles>

Major diastereomer of 2a

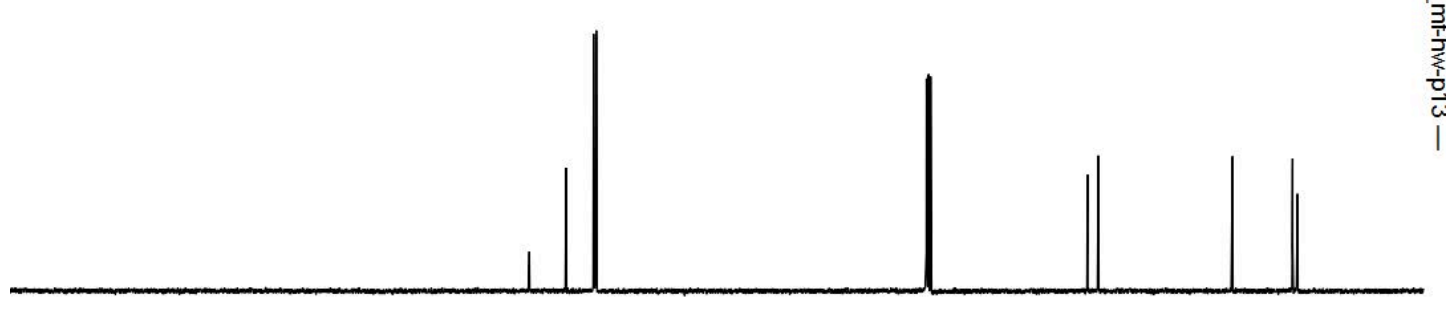

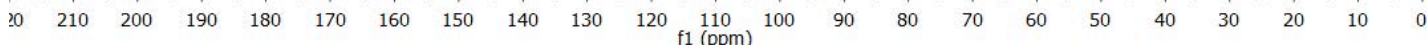

